Pollenanalytische Untersuchungen zur holozänen Vegetationsgeschichte entlang des östlichen unteren Odertals und südlichen unteren Wartatals in Nordwestpolen

\author{
Dissertation \\ zur Erlangung des Doktorgrades \\ der Mathematisch-Naturwissenschaftlichen Fakultäten \\ der Georg-August-Universität zu Göttingen
}

vorgelegt von

Christa Maria Herking

aus Epe

Göttingen 2004 

Danksagung .................................................................................................................................. 6

1. Einleitung...............................................................................................................................

2. Stand der Forschung ................................................................................................................ 8

3. Die Untersuchungsgebiete ..........................................................................................

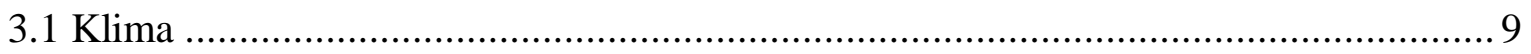

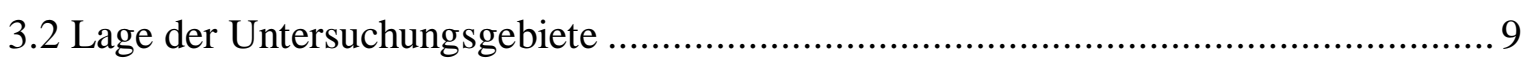

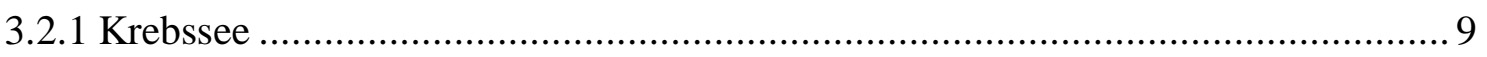

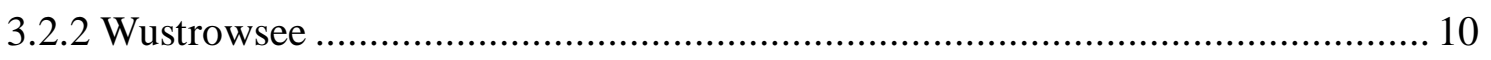

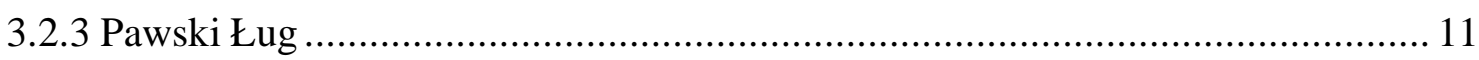

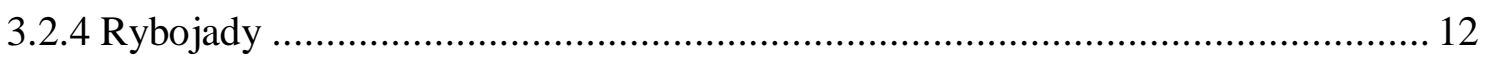

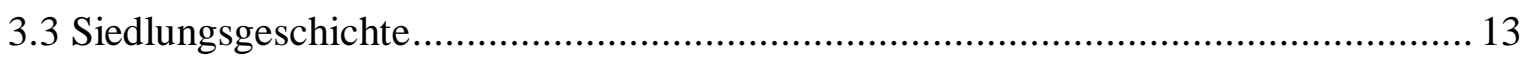

4. Material und Methoden................................................................................................... 23

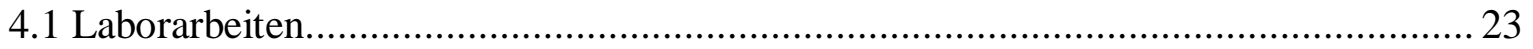

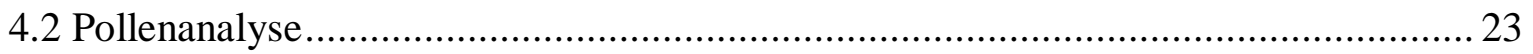

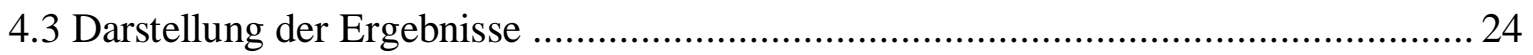

4.4 Sedimentstratigraphie vom Wustrowsee und Krebssee............................................ 25

4.5 Sedimentstratigraphie von Rybojady und Pawski Ług.............................................. 25

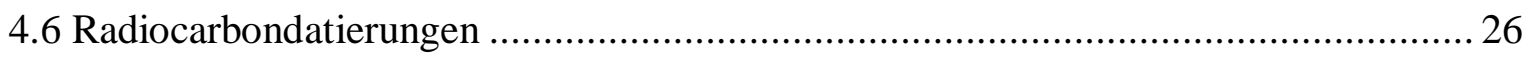

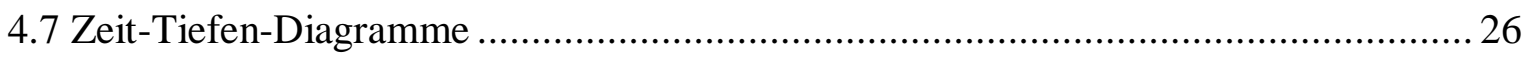

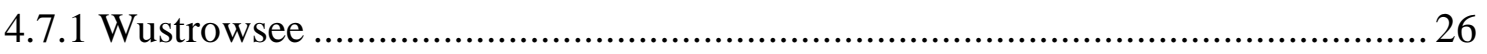

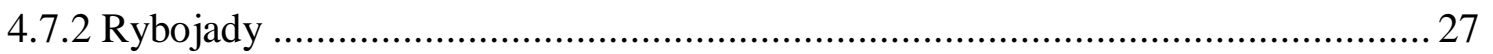

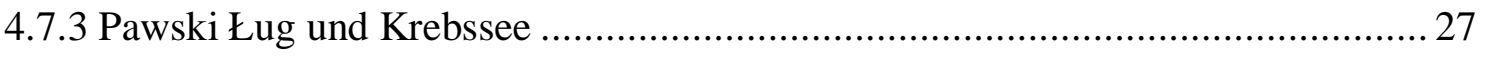

5. Diagrammbeschreibung ............................................................................................. 28

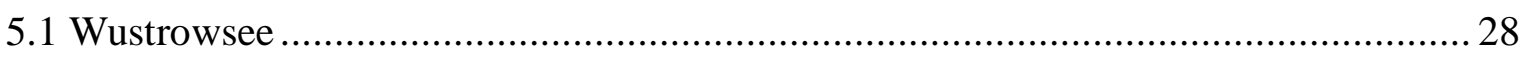

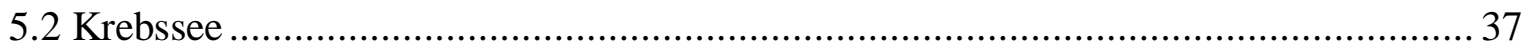

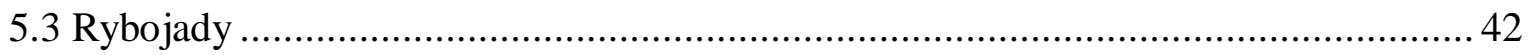

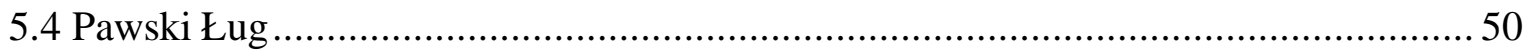

5.5 Synchronisation der lokalen Pollenzonen (L PAZ) mit waldgeschichtlichen

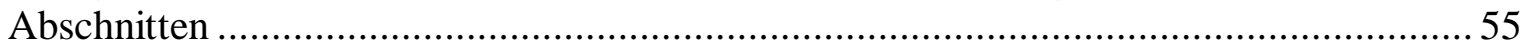

6. Die Vegetationsentwicklung entlang des östlichen unteren Odertals und südlichen unteren Wartatals..............................................................................................56

6.1 Die holozäne Vegetationsentwicklung am Wustrowsee und Krebssee ........................ 56

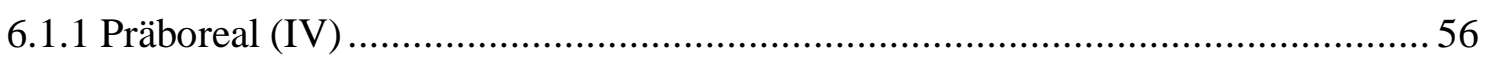




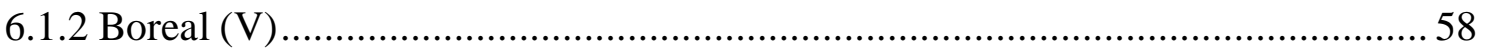

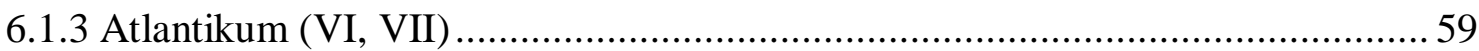

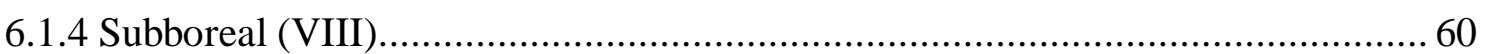

6.1.4.1 Der menschliche Einfluss auf die Vegetation im Neolithikum und in der

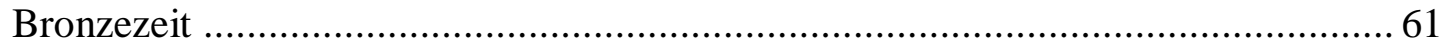

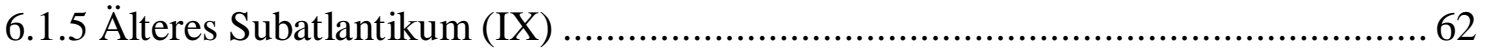

6.1.5.1 Der menschliche Einfluss auf die Vegetation von der Eisenzeit bis ins

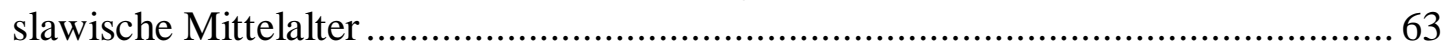

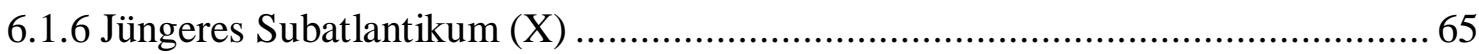

6.1.6.1 Der menschliche Einfluss auf die Vegetation im Mittelalter und in der Neuzeit

6.2 Die Vegetationsentwicklung im Umkreis von Rybojady und Pawski Ług 67

6.2.1 Das Problem der Ansprache des Spätglazials im Pollendiagramm von Rybojady 67

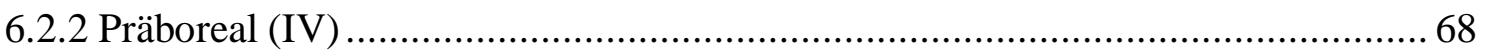

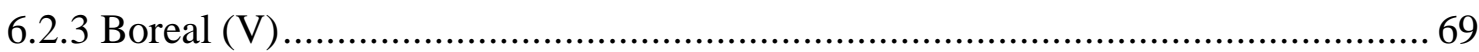

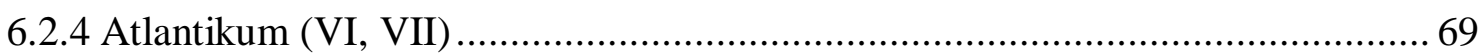

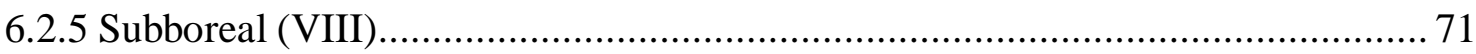

6.2.5.1 Der menschliche Einfluss im Neolithikum und in der Bronzezeit ................ 72

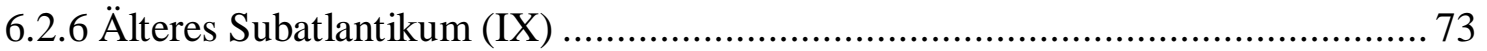

6.2.6.1 Der menschliche Einfluss auf die Vegetation von der Eisenzeit bis zum

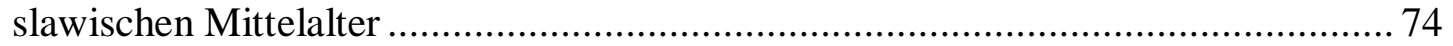

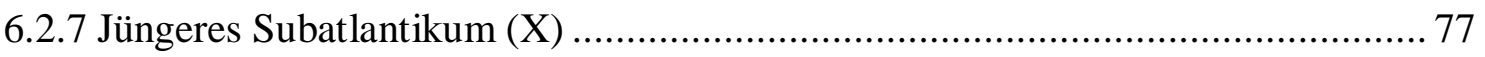

6.2.7.1 Der menschliche Einfluss auf die Vegetation im Mittelalter und in der Neuzeit

7. Der zeitliche Ablauf der holozänen Vegetationsentwicklung entlang des östlichen unteren Odertals und südlichen unteren Wartatals..................................................... 79

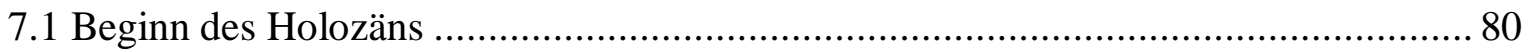

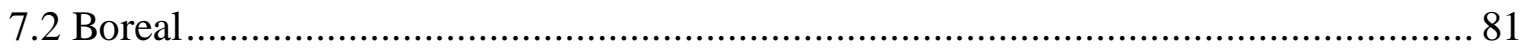

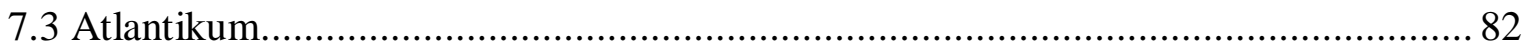

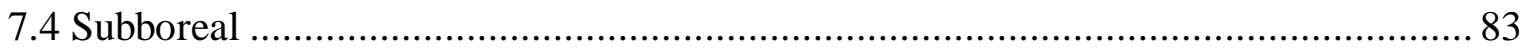

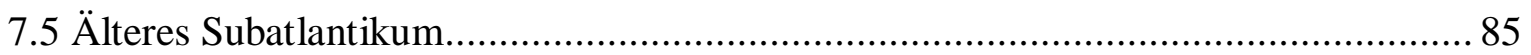

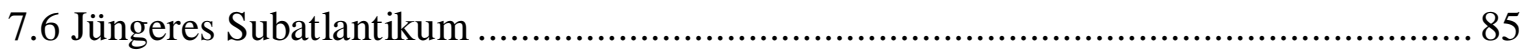

8. Zur Einwanderung und Ausbreitung von Fagus und Carpinus ............................. 86

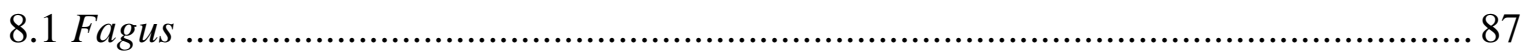

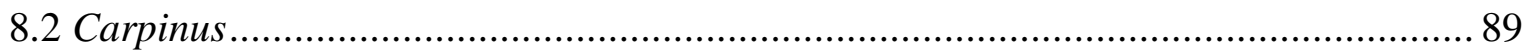

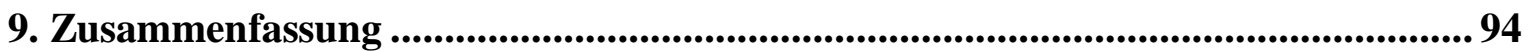

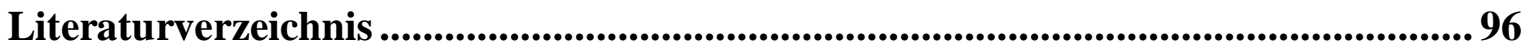




\section{Verzeichnis der Karten im Anhang}

Karte 1: Lage der Untersuchungsgebiete

Karte 2: Übersichtskarte zur Lage ausgewählter pollenanalytischer Arbeiten in Polen und Deutschland

Karte 3: Die geographische Lage vom Krebssee und Wustrowsee

Karte 4: Die geographische Lage von Pawski Ług

Karte 5: Die geographische Lage von Rybojady

\section{Verzeichnis der Abbildungen und Tabellen im Anhang}

Abbildung 1: Zeit-Tiefen-Diagramm vom Profil Wustrowsee

Abbildung 2: Zeit-Tiefen-Diagramm vom Profil Rybojady

Abbildung 3: Zeit-Tiefen-Diagramm vom Profil Pawski Ług

Abbildung 4: Zeit-Tiefen-Diagramm vom Profil Krebssee

Abbildung 5: Synchronisation der L PAZ mit den waldgeschichtlichen Abschnitten und den archäologischen Kulturstufen

Abbildung 6: Die Einwanderung und Ausbreitung von Fagus und Carpinus im Zusammenhang mit dem Corylus-Rückgang und der Zunahme von Siedlungszeigern

Tabelle 1: Sedimentstratigraphie vom Profil Wustrowsee

Tabelle 2: Sedimentstratigraphie vom Profil Krebssee

Tabelle 3: Sedimentstratigraphie vom Profil Rybojady

Tabelle 4: Sedimentstratigraphie vom Profil Pawski Ług

Tabelle 5: Meßdaten der Radiocarbonanalysen

Tabelle 6: Die lokalen PAZ vom Wustrowsee

Tabelle 7: Die lokalen PAZ vom Krebssee

Tabelle 8: Die lokalen PAZ vom Rybojady

Tabelle 9: Die lokalen PAZ vom Pawski Ług

Tabelle 10: Datierungen der Grenzen der waldgeschichtlichen Abschnitte

Tabelle 11: Dauer der waldgeschichtlichen Abschnitte

Tabelle 12: Die Datierungen des Ulmenfalles

Tabelle 13: Altersangaben zur Einwanderung und Ausbreitung von Fagus

Tabelle 14: Altersangaben zur Einwanderung und Ausbreitung von Carpinus 


\section{Verzeichnis der Beilagen}

Beilage 1: Pollen-Sporen-Algen-Prozentdiagramm Wustrowsee

Beilage 2: Pollen-Sporen-Algen-Prozentdiagramm Krebssee

Beilage 3: Pollen-Sporen-Algen-Prozentdiagramm Rybojady

Beilage 4: Pollen-Sporen-Algen-Prozentdiagramm Pawski Ług

Verzeichnis der verwendeten Abkürzungen:

$\begin{array}{ll}\text { A.D. } & \text { anno domini } \\ \text { AMS-Technik } & \text { accelerator mass spectrometry-Technik } \\ \text { B.C. } & \text { before christ } \\ \text { B.P. } & \text { before present (present =1950) } \\ \text { BP } & \text { Baumpollen } \\ \text { cal. } & \text { kalibriert } \\ \text { EMW } & \text { Eichenmischwald } \\ \text { FD } & \text { Feindetritus } \\ \text { HCL } & \text { Salzsäure } \\ \text { HF } & \text { Flusssäure } \\ \text { Indet. } & \text { Indeterminata } \\ \text { KD } & \text { Kalkdetritus } \\ \text { L PAZ } & \text { local pollen assemblage zone } \\ \text { NBP } & \text { Nichtbaumpollen } \\ \text { PAZ } & \text { pollen assemblage zone } \\ \text { PK } & \text { Pollenkörner } \\ \text { v.y. } & \text { varve years }\end{array}$




\section{Danksagung}

Mein besonderer Dank gilt Herrn Prof. Dr. Eberhard Grüger für die Betreuung der Arbeit. Des Weiteren danke ich den Mitarbeitern des Institutes für Biogeographie und Paläoökologie der Adam Mickiewicz Universität in Poznań, insbesondere Herrn Prof. Dr. Kazimierz Tobolski, für die tatkräftige Unterstützung bei den Geländearbeiten und die großzügige Bereitstellung von Literatur.

Herrn Prof. Dr. Eike Gringmuth-Dallmer verdanke ich die finanzielle Unterstützung durch die Volkswagenstiftung, mit der sowohl die Bohraktionen am Wustrowsee und Krebssee als auch die Radiocarbondatierungen durchgeführt werden konnten. Bei den Seebohrungen war die Arbeitsgruppe von Herrn Prof. Michael Walther vom Geographischen Institut der Freien Universität Berlin behilflich. Allen Beteiligten sei herzlichst gedankt; insbesondere Herrn Prof. Dr. Michael Walther, Herrn Dr. Frank Schlütz, Herrn Dipl. Geogr. Michael Krengel und Frau Marion Wassil sorgten für eine angenehme Arbeitsatmosphäre.

Für das gute Gelingen der Geländearbeiten in Zusammenhang mit der Bohrung in Rybojady und Pawski Lug danke ich Herrn Prof. Dr. Kazimierz Tobolski, Herrn Prof. Dr. Eberhard Grüger, Herrn Dr. Tomacz Schubert und Frau Dr. Ina Begemann.

Mein ganz besonderer Dank gilt Frau Viola Podsiadlowski, die in Kooperation mit dem brandenburgischen Landesamt für Denkmalpflege und dem Archäologischen Museum, insbesondere mit Frau Dr. Susanne Jahns, alle AMS- Proben und einen Teil der Pollenproben aufbereitet hat.

Wertvolle Tipps und Anregungen erhielt ich von Herrn Dr. Arthur Brande, Frau Dr. Susanne Jahns, Frau Dr. Ricarda Voigt, Herrn Dr. Frank Schlütz und Herrn Dr. Thilo Heinken.

Dem Deutschen Archäologischen Institut in Berlin danke ich sehr für die Bereitstellung eines Arbeitsplatzes. Für das entgegen gebrachte Verständnis und Vertrauen und die großzügige Freistellung von der Projektarbeit in den letzten Wochen vor Fertigstellung dieser Arbeit bedanke ich mich ganz besonders bei Herrn Dr. Walter Dörfler und Herrn Drs. Reinder Neef.

Schließlich möchte ich mich von Herzen bei all denen bedanken, die hier nicht namentlich erwähnt wurden, aber trotzdem zum Gelingen der vorliegenden Arbeit beigetragen haben. 


\section{Einleitung}

Die vorliegende Arbeit leistet einen Beitrag zur holozänen Vegetationsgeschichte entlang des östlichen unteren Odertals und entlang des südlichen unteren Wartatals. Auf der Basis von pollenanalytischen Untersuchungen an vier Standorten in Nordwestpolen (Karte 1) soll die postglaziale Vegetationsentwicklung rekonstruiert werden. Die lokal unterschiedlichen Entwicklungen werden diskutiert und mit Untersuchungen aus angrenzenden Gebieten verglichen. Besondere Beachtung gebührt der Einwanderung und Ausbreitung der Rotbuche, die in den hier untersuchten Gebieten aufgrund der zunehmenden Kontinentalität an die Grenzen ihres Herrschaftsbereiches stößt.

Die Idee zur vorliegenden Arbeit entstand im Jahre 1999 während meiner Tätigkeit als Palynologin in dem interdisziplinären, von der Volkswagen-Stiftung gefördertem Projekt „Mensch und Umwelt im Odergebiet in ur- und frühgeschichtlicher Zeit“ am Deutschen Archäologischen Institut in Berlin. Innerhalb der Projektzeit wurden entlang des westlichen unteren Odertals, beginnend mit der Ueckermünder Heide im Norden bis zur Niederlausitz im Süden insgesamt sechs Lokalitäten pollenanalytisch bearbeitet, die die erwarteten Unterschiede der postglazialen Vegetationsentwicklung entlang des gewählten Nord-Süd-Gradienten dokumentieren. Insbesondere belegen sie die mit zunehmender Kontinentalität abnehmende Konkurrenzkraft der Rotbuche in diesem Gebiet. Um festzustellen, wie weit das Herrschaftsgebiet der Rotbuche über das westliche untere Odertal hinaus in Richtung Osten reicht, fehlten bislang moderne hochauflösende und gut datierte pollenanalytische Untersuchungen zur holozänen Vegetationsentwicklung aus dem östlichen unteren Odertal und dem angrenzenden unteren Wartatal. Ein Teil dieser Forschungslücke soll mit der vorliegenden Arbeit geschlossen werden. 


\section{Stand der Forschung}

Eine Vielzahl früher Arbeiten zur Waldentwicklung im ostpommerschen Endmoränengebiet stammt aus den dreißiger Jahren des zwanzigsten Jahrhunderts, darunter HESMER (1931), HESMER (1932) und NiETSCH (1934). NIETSCH untersuchte vor allem Moore und verlandete Seen im Gebiet der fruchtbaren Schwarz- und Braunerdeböden südöstlich von Szczecin, darunter das Gebiet um Pyrzyce, auch Pyritzer Weizacker genannt, das siedlungsgeschichtlich von besonderem Interesse ist, da es während des Neolithikums stark besiedelt war. HESMER (1932) stellte in seinen pollenanalytischen Untersuchungen aus dem Grundmoränengebiet der Neumark die standörtlich unterschiedliche Waldentwicklung heraus. Er dehnte seine Untersuchungen auf die östliche Mark Brandenburg aus und führte u.a. Pollenanalysen an einem Standort nahe Łagów durch (HESMER 1933); hier stellte er eine unerwartet hohe Rotbuchenpräsenz fest. Sehr detaillierte und datierte vegetationsgeschichtliche Untersuchungen liegen von der Insel Wolin vor (LATAŁOWA 1992; 2001a). In Richtung Süden schließen sich daran die Untersuchungen von BOROWKA et al. (2001) in Szczecin und im Gebiet innerhalb der pommerschen Seenplatte im Drawa Nationalpark diejenigen von LATAŁOWA (2001b) an. Besonders viele vegetationsgeschichtliche Forschungen konzentrieren sich in der großpolnisch-kujawischen Ebene, im Umkreis von Poznań und Gniezno (Karte 2). Wie die Arbeiten von TOBOLSKI (1990; 1991), ToBOlski \& LitT (1994), MAKOHONIENKo (2001b) und MiLECKA (1998; 2001a) zeigen, zählt der mehrere Seen umfassende Lednica Landschaftspark nordöstlich von Poznań zu einem intensiv untersuchten Gebiet. LiTT (1988) befasste sich vor allem mit der spätglazialen Vegetationsentwicklung im Lednica Landschaftspark. Ein besonders detailliertes Bild der Vegetationsentwicklung in Zentralpolen der letzten 15.000 Jahre liefern die interdisziplinären Untersuchungen an den laminierten Sedimenten des GościążSees (RALSKA-JASIEWICZOWA et al. 1992), (RALSKA-JASIEWICZOWA et al. 1998)(vgl. Karte 2). 


\section{Die Untersuchungsgebiete}

Der Krebssee und der Wustrowsee liegen im östlichen unteren Odertal, im sogenannten Oderknie. Das Rybojady-Moor und das Pawski Ług-Moor befinden sich im südlichen unteren Wartatal (Karte 1). Im Folgenden wird zunächst das Klima des Großraumes beschrieben. Die genaue Lage sowie ausgewählte physisch-geographische und vegetationskundliche Informationen werden in den Beschreibungen der einzelnen Untersuchungsgebiete zusammengefasst.

\subsection{Klima}

Die vier Untersuchungsgebiete (Karte 1) liegen im Übergangsbereich der maritim geprägten zu den kontinental geprägten Mittelbreiten. Die Jahresmitteltemperatur beträgt ca. $8{ }^{\circ} \mathrm{C}$ - die durchschnittliche Temperatur des wärmsten Monats (Juli) ca. $18^{\circ} \mathrm{C}$, die des kältesten Monats (Januar) ca. $-1,5{ }^{\circ} \mathrm{C}$ (vgl. BorowKA et al. 2004). Die Anzahl der Frosttage beläuft sich auf durchschnittlich 55-75 Tage im Jahr. Im Jahresmittel fallen ca. $500 \mathrm{~mm}$ bis $600 \mathrm{~mm}$ Niederschlag. Die Niederschlagsbilanz ist ganzjährig positiv (WALTER \& LIETH 1960-1967). Der überwiegende Teil fällt während der Vegetationsperiode in den Sommermonaten. Eine geschlossene Schneedecke liegt in den Untersuchungsgebieten Wustrowsee und Krebssee an etwa 20-40 Tagen im Jahr vor. Weiter südöstlich, nahe den Untersuchungsgebieten Pawski Ług und Rybojady, wurde eine durchschnittlich länger andauernde Schneebedeckung an etwa 40-60 Tagen im Jahr ermittelt (LESZCZYCKI 1973-1978).

Von wesentlicher Bedeutung sind kleinräumige, d.h. mikroklimatische Differenzierungen aufgrund des Reliefs in unmittelbarer Umgebung der Untersuchungsgebiete. Auf die Bedeutung der Bildung von Kaltluftseen und die Veränderung klimatischer Faktoren in Abhängigkeit vom Wasserstand bzw. Seespiegel ist an dieser Stelle beispielhaft hinzuweisen. Detaillierte mikro- und mesoklimatische Untersuchungen liegen für die hier untersuchten Gebiete bisher jedoch nicht vor.

\subsection{Lage der Untersuchungsgebiete}

\subsubsection{Krebssee}

Der Krebssee (Jezioro Skorupiaki) $\left(52^{\circ} 53\right.$ N $\left./ 14^{\circ} 16^{`} \mathrm{E}\right)$ liegt in einer flachkuppigen, mäßig bewaldeten weichselzeitlichen Grundmoränenlandschaft (WOLDSTEDT 1974) des 
Pommerschen Stadiums zwischen Pommerscher Eisrandlage im Süden und der Angermünder bzw. Chojna Staffel im Norden (LIEDTKE 1981; MARKS 2002).

Er befindet sich ca. $1 \mathrm{~km}$ südwestlich der Gemeinde Orzechów nahe der Hauptstraße 124 (Karte 3). Seine Wasserfläche beträgt etwa $0,36 \mathrm{~km}^{2}$, die maximale Wassertiefe ca. $14 \mathrm{~m}$. Die direkten Einzugsgebiete werden rezent durch den Anbau von Weizen und Roggen überwiegend ackerbaulich genutzt, während die Weidewirtschaft deutlich in den Hintergrund tritt. Die Ufervegetation besteht aus einem den See fast komplett umfassenden Schilfröhricht, welches sich überwiegend aus Phragmites australis und Typha angustifolia zusammensetzt. Am Nord- und am Westufer ist das Röhricht sehr breit, an den übrigen Uferbereichen ist der Seeboden relativ schnell abfallend, so dass hier nur ein schmaler Schilfsaum ausgebildet ist.

An den Schilfgürtel schließt ein lockerer Uferwald an. Größere Waldbestände gibt es in der unmittelbaren Nähe nicht. Relativ häufig findet man rundliche kleine, abflusslose Kessel, die sogenannten Sölle, die meist umgeben sind von einem schmalen Saum aus Salix, Alnus und Corylus. Der den See umgebene Uferwald setzt sich aus folgenden Arten zusammen (die Reihenfolge entspricht der Häufigkeit):

Salix alba, Salix caprea, Fraxinus exelsior, Alnus glutinosa, Quercus robur, Populus alba, Sambucus niger, Robinia pseudacacia, Corylus avellana, Carpinus betulus, Crataegus monogyna, Populus nigra, Acer pseudoplatanus und Ulmus glabra.

\subsubsection{Wustrowsee}

Der Wustrowsee (Jezioro Ostrow) $\left(52^{\circ} 56\right.$ N $\left./ 14^{\circ} 19^{\prime} \mathrm{E}\right)$ liegt in einer kuppigen, überwiegend dicht bewaldeten weichselzeitlichen Grundmoränenlandschaft im Übergangsbereich zum sich südlich anschließenden Endmoränenzug der Eisrandlage der Angermünder bzw. Chojna Staffel (Karte 3) (LIEDTKE 1981; MARKS 2002).

Wesentliches sedimentologisches und geomorphologisches Merkmal dieses Raumes ist eine enge Verzahnung kleinerer Sander-, Oser- und Kamesflächen mit Bereichen, die stärker durch anstehendes moränisches Substrat gekennzeichnet sind (EHLERS 1994; WOLDSTEDT 1974). Das Relief ist stark zergliedert und weist eine für diesen Großraum relativ hohe Reliefenergie auf. Entsprechend ist mit einer kleinräumigen Veränderung der Standortbedingungen zu rechnen. 
Der See befindet sich ca. $8 \mathrm{~km}$ südwestlich der Stadt Chojna, nahe der Ortschaft Stoki (Karte 3).

Es ist ein in SW-NO ausgerichteter See mit einer Wasserfläche von ca. 0,9 $\mathrm{km}^{2}$ und einer maximalen Wassertiefe von ca. $10 \mathrm{~m}$. An das südliche Ufer schließt ein Schwarzerlenbruch an, das nördliche und westliche Ufer werden von einem lockeren und relativ schmalen Schilfröhricht eingenommen. Auf dem See, Richtung Ostufer, befindet sich eine kleine, mit Erlen und Weiden bestockte Insel, die wiederum von einem breiten Schilfröhricht umgeben ist. Dieses Röhricht ist auf der Ostseite der Insel so breit, dass es die Insel mit dem östlichen Seeufer verbindet. Rings um den See wächst mit Ausnahme des Bruchwaldes im Süden ein relativ breiter, sehr abwechslungsreicher Laubmischwald, der dann an einen einheitlichen Kiefernforst anschließt. Inmitten dieses Laubmischwaldgürtels ist nordwestlich des Sees inselartig ein frischer Kalkbuchenwald mit Mercurialis perennis und Galium odoratum ausgebildet. Der übrige Laubmischwald setzt sich überwiegend aus folgenden Gehölzen zusammen:

Quercus petraea, Quercus robur, Betula pubescens, Fagus sylvatica, Alnus glutinosa, Fraxinus excelsior, Pinus sylvestris, Frangula alnus, Corylus avellana, Acer pseudoplatanus, Quercus rubra, Ulmus glabra, Prunus spinosa, Picea abies, Sorbus aucuparia, Tilia cordata, Aesculus hippocastanum.

\subsubsection{Pawski Ług}

Das Moor (52 $\left.19^{`} \mathrm{~N} / 15^{\circ} 19^{\prime} \mathrm{E}\right)$ mit einer Größe von ca. 0,018 km² liegt westlich der Straße, die nahe der Siedlung Pozrzadło von der Hauptstraße E30 nach Norden Richtung Miedzyrzecz abbiegt, ca. 2 km vor dem Ort Łagów (Karte 4) in einer rezent abflusslosen Mulde. Die Mulde liegt in einer flachkuppigen Grundmoränenlandschaft zwischen dem Endmoränenzug der Pommerschen Phase im Norden und der Eisrandlage des Frankfurter bzw. des Poznań Stadiums im Süden (LIEDTKE 1981; Marks 2002). Das Relief im Untersuchungsgebiet ist kleinräumig in Kuppen und Mulden gegliedert und weist Höhenunterschiede im niedrigen Dekameterbereich auf. Das Moor kann als verlandeter Teil einer vorzeitlich größeren geschlossenen Wasserfläche interpretiert werden und liegt im Zwickel zwischen zwei Hauptentwässerungslinien.

Nach der Moorcharakterisierung von SuCcow (2001) handelt es sich um ein Zwischenmoor mit einer typischen Zwischenmoor-Gesellschaft (Sphagnum magellanicum, S. rubellum, Eriophorum vaginatum, E. angustifolium, Vaccinium oxycoccus). In den 
Randbereichen und auch auf der Moorfläche finden sich Moorbirken und Kiefern, die, nach Erreichen einer Wuchshöhe von maximal 3 m, zum größten Teil verkümmert sind.

Die Ergebnisse der vegetationskundlichen Untersuchung des in einem mehrere ha großen Laubmischwald eingebetteten Zwischenmoores zeigen eine deutliche Dominanz von Rotbuche und Traubeneiche. Der Wald ist gegliedert durch Mulden mit Durchmessern und Tiefen in Meter-Dekameter-Bereichen. Insbesondere in den Sattelzonen zwischen den Mulden finden sich vereinzelt alte Wald-Kiefern, mit einem Stammdurchmesser bis zu $80 \mathrm{~cm}$. Des weiteren sind in Moornähe Vaccinium myrtillus, Rhamnus frangula, Dryopteris carthusiana und Thelypertis palustris häufig vertreten.

\subsubsection{Rybojady}

Das $0,08 \mathrm{~km}^{2}$ große Rybojady-Moor $\left(52^{\circ} 24\right.$ N $\left./ 15^{\circ} 49^{\prime} \mathrm{E}\right)$ liegt etwa $25 \mathrm{~km}$ östlich von Miedzyrzecz, in der Nähe von Pszczew, im Pszczewski Landschaftspark (Karte 5) inmitten eines mehrere Hektar großen Kiefernforstes. Die rezent abflusslose Mulde ist geomorphologisches Element der auf der Basis weichselzeitlicher glazifluvialer Binnensanderflächen entstandenen postglazialen Landschaft (WOLDSTEDT 1974). Das Relief ist flachkuppig mit nur mäßigen Höhenunterschieden von wenigen Metern (Karte 5). Der Vergleich mit den Untersuchungsgebieten Wustrowsee und Pawski Ług verdeutlicht den unterschiedlichen Relieftyp (Karte 3 und Karte 4). Die sedimentologischen und geomorphologischen Standortbedingungen der letztgenannten Gebiete sind erheblich stärker differenziert als am Rybojady-Moor.

Nach der Moortypologie von SuCCOW (2001) handelt es sich um ein Zwischenmoor. Die Vegetation auf dem Moor besteht zum größten Teil aus Sphagnum magellanicum, S. rubellum, Eriophorum angustifolium, Vaccinium oxycoccus, Andromeda polifolia, aus verschiedenen Carex-Arten, u.a. Carex rostrata und C. nigra sowie aus Menyanthes trifoliata. An den Moorrändern und vereinzelt auch auf dem Moor wachsen kleine, maximal 1,5 m hohe Kiefern und Birken.

Rund um das Moor, in dem Bereich, in dem die Mulde sanft ansteigt, befindet sich ein feuchter Laubwaldsaum, der sich überwiegend aus Alnus glutinosa, Betula pubescens, Salix alba und Rhamnus frangula zusammensetzt. Am südlichen Rand des Moores ist dieser Saum verbreitert, und es hat sich hier ein kleines Erlenbruch entwickelt. 


\subsection{Siedlungsgeschichte}

Die Wiederbesiedlung des nordmitteleuropäischen Flachlandes begann im Spätglazial, in der Spätphase der weichselzeitlichen Vereisung. Diese Periode war durch eine ausgesprochene Klimadynamik gekennzeichnet, die sich nicht nur in der Vegetationsentwicklung, sondern auch in der Siedlungsgeschichte widerspiegelt. Im Folgenden werden - beginnend mit dem Paläolithikum - die siedlungsgeschichtlichen Grundzüge entlang des unteren Odertals und entlang des mittleren Wartatals vorgestellt.

\section{Paläolithikum}

Die ältesten archäologischen Funde stammen aus dem Spätpaläolithikum. Es handelt sich dabei vor allem um Artefakte aus Knochen und Geweih - Hinterlassenschaften von Rentierjägern, die der sogenannten Hamburger Kultur zugeordnet werden (KoBUSIEWICZ et al. 1987). Archäologisches Fundmaterial aus dieser Zeit ist im entfernten Umfeld der Untersuchungsgebiete, im mittleren und unteren Odergebiet, weit verbreitet. Aus der direkten Umgebung vom Wustrow- und Krebssee sowie aus dem nahen Umkreis von Łagów und Rybojady sind jedoch keine paläolithischen Funde bekannt (GRAMSCH 2002: Abb. 113). Die kartierten Fundplätze konzentrieren sich eher im Bereich feuchter Niederungen in den großen Urstromtälern. Hochflächen wurden offensichtlich gemieden (WECHLER 1996). Wie überall in der endpleistozänen Umwelt der nordeuropäischen Ebene zeigten die paläolithischen Jäger-Sammler-Kulturen unterschiedliche Anpassungsweisen an ihre Umwelt, die einerseits aus einer Parktundra, anderseits aus lichten Kiefernwäldern bestand (LANG 1994; KOBUSIEWICZ 2002). Infolge der Klimaänderung und der damit verbundenen Vegetationsveränderungen folgte ein Teil der an Rentierjagd angepassten spätpaläolithischen Bevölkerung der in die bevorzugten Lebensräume nach Norden abwandernden Jagdbeute. Der übrige Teil der altsteinzeitlichen Populationen blieb und stellte sich auf die neuen ökologischen Bedingungen ein (KOBUSIEWICZ 2002).

\section{Mesolithikum}

Die Übergangsperiode zwischen ausgehender Alt- und beginnender Jungsteinzeit, das Mesolithikum, gliedert sich in einen älteren und einen jüngeren Teil. Der ältere Abschnitt entspricht den postglazialen Perioden Präboreal und Boreal, der jüngere dem Atlantikum. Durch die Landbrücken, die noch während des älteren Mesolithikums bestanden, entstand eine England, Skandinavien, Norddeutschland, Pommern und Großpolen umfassende kulturelle Einheit, die Maglemose-Kultur, die auch die Gebiete entlang der unteren und 
mittleren Oder umfasste. Aus der direkten Umgebung der Untersuchungsgebiete sind keine archäologischen Fundstücke bekannt, jedoch liegen etwa $20 \mathrm{~km}$ westlich vom Krebssee, u.a. aus den Kreisen Angermünde und Eberswalde, zahlreiche typische Kleingeräte, mesolithische Mikrolithe, vor (GRAMSCH 2002: Abb. 115). Aus der Verteilung der Funde lässt sich ableiten, dass Siedel- und Lagerplätze erneut bevorzugt in Gewässernähe, vor allem im Odermündungsgebiet angelegt wurden. Dauerwohnplätze hat es im Mesolithikum noch nicht gegeben (GRAMSCH 2002).

Die Menschen bestritten ihren Lebensunterhalt weiterhin durch Jagen, Sammeln und Fischen, wobei das Spektrum der Jagdgeräte und die Ausnutzung der natürlichen Ressourcen breiter waren als zuvor. Pollenanalytisch ist der Einfluss des Menschen auf die Vegetation zu dieser Zeit jedoch noch nicht erfassbar.

\section{Neolithikum}

Das Neolithikum kennzeichnet den Übergang von der aneignenden Wirtschaftsweise mit Jagen, Sammeln und Fischen zur produktiven Wirtschaftsweise in Form von Ackerbau und Viehzucht. Der Beginn dieser agrarischen Wirtschaftsweise ist im Odergebiet mit der Bandkeramik, die sich von Südosteuropa ausgehend Richtung Nordwesten ausbreitete, und mit der Rössener Kultur verbunden. Aus der weiteren Umgebung des Untersuchungsgebietes sind im Bereich der fruchtbaren Grundmoränenböden zwei bandkeramische Siedlungskammern, die Uckermark westlich und der Pyritzer Weizacker östlich der unteren Oder, bekannt (WECHLER 1996). Aus der direkten Umgebung der Untersuchungsgebiete fehlen bisher die Hinweise auf frühneolithische Siedler.

Weniger fruchtbare Gebiete wurden im Laufe der Zeit von der Bevölkerung der Trichterbecherkultur eingenommen. Diese Kultur ist vermutlich aus ehemals mesolithisch lebenden Bevölkerungsgruppen im nord-mitteleuropäischen Raum entstanden, die allmählich die Grundlagen der produzierenden, neolithischen Wirtschaftsweise übernahmen. Der bevorzugte Siedlungsraum wechselte von flussnahen, oft sumpfigen Arealen zu etwas trockeneren Regionen. Auf diese Weise entstanden u.a. entlang der unteren Oder, an der Warta (Warthe) und an der Noteć (Netze) kleine Siedlungsagglomerationen. Sie waren nicht so stabil wie die Ansiedlungen auf den fruchtbaren Grundmoränenböden und hatten eher den Charakter kurzzeitig aufgesuchter, saisonaler Lager (KULCZYCKALECIEJEWICZOWA 2002). In der Nähe des Krebssees und des Wustrowsees sind zwei Fundstellen bekannt, die archäologische Funde der Trichterbecherkultur erbrachten (KULCZYCKA-LECIEJEWICZOWA 2002: Abb. 117). 
Gegen Ende des Neolithikums erreichten Bevölkerungsgruppen der Schnurkeramik-Kultur das Untersuchungsgebiet. Ihre Lagerplätze waren in der Regel keine dauerhaften Siedlungsplätze, so dass kaum archäologisches Fundmaterial aus schnurkeramischen Siedlungen vorliegt. Besser bekannt sind die Gräber der Schnurkeramiker. Nördlich von Łagów und Rybojady liegt eine Fundstelle, die dieser Kultur zugewiesen wird (KULCZYCKA-LECIEJEWICZOWA 2002: Abb. 118).

Während des Neolithikums existierten die neolithische und die mesolithische Wirtschaftsweise nebeneinander. Die Mesolithiker nutzten vor allem die Überschwemmungsgebiete der Flüsse sowie die Sumpfgebiete und lebten weiter vom Fischfang, von der Jagd und der Sammelwirtschaft. Nach KuLCZYCKA-LECIEJEWICZOWA (2002) sind mesolithisch lebende Bevölkerungen bis in die Bronzezeit hinein nachweisbar.

\section{Bronzezeit}

Während der Bronzezeit war die Kupfergewinnung von großer Bedeutung. Ausgehend von den führenden Zentren der Metallverarbeitung im Südosten Europas verbreiteten sich die Techniken des Bronzegusses Richtung Nordeuropa. Gegenstände aus Bronze galten als Prestigeobjekt, als Zeichen für Macht und Ansehen und als sichtbares Zeichen für Wohlstand. Gräber von Metallurgen deuten auf eine sozial herausgehobene Position, die auch auf die sich herausbildende stärkere gesellschaftliche Gliederung hinweist.

Die Entwicklung und Verbreitung des Kupferbergbaus und der metallverarbeitenden Techniken setzten weitreichende Handels- und Verkehrsbeziehungen der verschiedenen Bevölkerungsgruppen voraus. Trotz der Vielzahl von archäologischen Funden ist der Kenntnisstand über die kulturellen Beziehungen noch unbefriedigend. Große Probleme bereiten den Forschern der noch unzureichende Kenntnisstand über die Keramik sowie das Fehlen von nachgewiesenen Siedlungsplätzen, denn die „barbarischen“ Gesellschaften des bronzezeitlichen Europas errichteten zwar prunkvolle, bis zu 8,5 m hohe Hügelgräber mit z. T. wertvollen Grabbeigaben aus Gold und Bronze, andere Bauwerke, Siedlungen sowie Gebrauchsgegenstände waren jedoch bescheiden (SHERRATT 1998).

Auf Grund der stilistisch-typologischen Unterschiede der materiellen Hinterlassenschaften wird der Ablauf der nord-mitteleuropäischen Bronzezeit in verschiedene Perioden (I-V) eingeteilt.

Während der frühen Bronzezeit (Periode I) gehörte das östliche Odergebiet zum Hauptverbreitungsgebiet der Aunjetitzer Kultur, innerhalb derer sich mehrere verschiedene 
Lokal- und Regionalgruppen differenzieren lassen (KŁOSINSKA 1996; WESOŁOWSKI 1996; GEDIGA \& MiERZWINSKI 2002). In der Periode II strahlten die Einflüsse der Vorlausitzer Kultur, die überwiegend in Ober- und Niederschlesien sowie in Großpolen vertreten war, in die Gebiete entlang der Oder aus. Im Laufe der mittleren Bronzezeit (Ende Periode II, Periode III) fand im gesamten Oderraum eine Besiedlungszunahme sowie ein Kulturwandel statt. Massive Veränderungen innerhalb der Wirtschafts- und Gesellschaftsstrukturen, einerseits beeinflusst durch Kulturen des östlichen Karpatenbeckens und des Nordwestbalkan, anderseits aus dem sog. Nordischen Kreis, kennzeichnen diesen Zeitabschnitt.

Einer der wichtigsten Aspekte des geistigen Wandels war eine Veränderung innerhalb des spirituell-religiösen Lebens, der sich in dem überregionalen Übergang von der Körperbestattung zur Brandbestattung (sog. Urnenfelderzeit) widerspiegelt und im Odergebiet durch die seit dem 14. Jh. v. Chr. nachgewiesene Lausitzer Kultur repräsentiert wird. Die Leichen wurden verbrannt und der in Urnen gesammelte Leichenbrand wurde in Gräberfeldern beigesetzt. Im Laufe der jüngeren Perioden der Bronzezeit entwickelten sich lokale Varianten der Lausitzer Kultur, die bis in die frühe Eisenzeit das Odergebiet besiedelten.

Bis zum 8. Jh. v. Chr. war das Untersuchungsgebiet relativ dicht besiedelt. Statt der leichten, durchlässigen Böden wurden nun die guten, relativ schweren Böden der Grundmoränenflächen als Siedlungsplätze genutzt. Die Besiedlung konzentrierte sich auf jene Regionen, in denen beste Braunerden vorhanden waren. Dies waren u. a die Gebiete um Szczecin, Wolin, Pyrzyce und Cedynia. Möglicherweise steht dies in Zusammenhang mit der zunehmenden Bedeutung des Ackerbaus in der Nahrungsmittelproduktion.

Einen Überblick über die Verteilung bronzezeitlicher Besiedlungsspuren (Siedlungen, Gräber und Hortfunde) verschiedener Perioden liefert MIERZWINSKI (1996: Abb. 2, Abb. 3, Abb. 4). Auch aus der Umgebung der Untersuchungsgebiete sind bronzezeitliche Funde bekannt (GEDIGA \& MIERZWINSKI 2002).

Gegen Ende der Bronzezeit breiteten sich von Süden kommend über Ober- und Niederschlesien Einflüsse der Hallstattkultur im Odergebiet, etwa bis zur Noteć, aus. Die Bewohner der weiter nördlich gelegenen Gebiete des unteren und mittleren Oderlaufs pflegten mehr Kontakt zum Nordischen Kreis, so dass nördlich der Noteć und der Warta nur sehr wenige archäologische Funde mit südlichen Anklängen vorliegen (GEDIGA \& MIERZWINSKI 2002: Abb. 123). Unter dem Einfluss der Hallstattkultur entwickelten sich regional verschiedene früheisenzeitliche Gruppen. Gesellschaftliche Spannungen 
innerhalb der Stammesgesellschaften führten vielerorts zur Gründung von Befestigungsanlagen sowie zur Errichtung von Höhen- und Niederungsburgen mit Palisadenringen, die in Krisenzeiten als Schutzburgen dienten, z. B. die befestigte Burganlage in Biskupin, Zentralpolen (WESOŁOWSKI 1996; HARDING 1998).

\section{Eisenzeit}

Der Beginn der Vorrömischen Eisenzeit an der Wende vom Subboreal zum Subatlantikum ist durch einen das gesamte nordeuropäische Tiefland betreffenden Klimawandel gekennzeichnet. Dieser ist durch ein Absinken der Jahresmitteltemperatur und einen Anstieg der Niederschläge charakterisiert (BROSE \& HEUBNER 2002). Unabhängig von den klimatischen Änderungen setzte bereits vor dem Beginn der Eisenzeit eine Epoche grundlegender gesellschaftlicher Umorientierung ein. Erst allmählich setzte sich die Eisenmetallurgie durch, so dass erst ab etwa 600 v. Chr. die meisten Werkzeuge und Waffen nicht mehr aus Bronze, sondern aus Eisen hergestellt wurden. Hochwertige Prestigegegenstände fertigte man dagegen weiterhin aus Bronze (HARDING 1998).

Während der vorrömischen Eisenzeit war im Odergebiet vor allem die Göritzer Gruppe, eine Regionalgruppe der in der Bronzezeit entstandenen Lausitzer Kultur verbreitet. Eine Reihe von unbefestigten Siedlungen und Gräberfunden ist aus der Nähe von Krebs- und Wustrowsee bekannt (LEUBE 1996: Abb. 1). Zu den eindrucksvollsten archäologischen Befunden der Göritzer Kultur gehören die Burgen von Lossow und Lebus an der mittleren Oder (SCHRAGE 1995).

In der jüngeren vorrömischen Eisenzeit, d. h. im ausgehenden 5.-3. Jh. v. Chr., brachen die Besiedlungsspuren der Göritzer Gruppe ab. An ihre Stelle traten germanische Stammesgruppen. Während der Latènezeit unterlag die Bevölkerung dem Einfluss der Przeworskund Jastorfkultur (LEWCZUK 2002: Abb. 125, 126).

Insgesamt herrschte während der vorrömischen Eisenzeit eine starke negative Bevölkerungsdynamik, so dass bereits um 500 v. Chr. große Teile des Odergebietes entvölkert waren.

\section{Römische Kaiserzeit und Völkerwanderungszeit}

Die Bevölkerungsentwicklung im Laufe der römischen Kaiserzeit verlief regional unterschiedlich. $\mathrm{Zu}$ Beginn dieser Phase wurde bevorzugt das Odermündungsgebiet besiedelt, während in der jüngeren römischen Kaiserzeit sich die Siedlungen allmählich von Norden her in die südlicheren Gebiete verlagerten (LEUBE 1991). Dort lebende 
Bevölkerungsgruppen zeichneten sich durch eine spezielle Art der Metallverarbeitung und eine auf Drehscheiben gefertigte Keramik aus (SCHULTZE 1998).

Der gesamte Oderraum stellte ein Grenzland für verschieden große Kulturkomplexe dar, so dass nicht ganz eindeutig ist, in wieweit eine kulturelle Einflussnahme der aus Westen und Osten einwandernden Bevölkerungen oder ein Bevölkerungswechsel bzw. eine Kombination von beidem vonstatten ging (DOMANSKI et al. 2002). Das Vorkommen römischer Importwaren, wie Metallgefäße, Münzen, Schmuckgegenstände und Waffen, charakterisiert das Odergebiet als einen gut funktionierenden, überregional bedeutsamen Wirtschaftsraum, wobei ein Teil der Importwaren vermutlich als Geschenke oder Beute ins Gebiet kam.

Westlich des mittleren und unteren Oderlaufs lassen sich von Norden nach Süden vier verschiedene Siedlungsgebiete differenzieren, wovon zwei, das Eberswalder Urstromtal/Oderbruch-Gebiet mit der Oderbruch-Gruppe sowie die Ostbrandenburgische Platte mit der Buckow-Lebuser-Gruppe für die Untersuchungsgebiete relevant sind. Neben der Oderbruch-Gruppe und der Buckow-Lebuser-Gruppe sind darüber hinaus aus dem Gebiet östlich der Oder archäologische Funde der Wielbark-Kultur und der Luboszyce-Kultur bekannt.

Bevorzugte Siedlungsplätze dieser Zeit waren sowohl die feuchten Talsandflächen der Urstromtalniederungen der Oder, der Warta und der Noteć als auch ehemalige Uferwälle und Sandinseln. Die trockenen Sanderflächen, die lehmigen Sande der Ostbrandenburgischen Platte und die Uferzonen der tief eingeschnittenen Rinnenseen blieben unbesiedelt (LEUBE 1996). Einen Einblick in die dorfartigen Siedlungsstrukturen, die eine gewisse Planung voraussetzten, gewähren die spätkaiser- und frühwanderungszeitlichen Siedlungen von Frankfurt-Kliestow und Wüste Kunersdorf. Östlich der Oder ist aus dem NeißeWodra-Gebiet eine Reihe vergleichbarer Siedlungen bekannt (LEUBE 1996; DOMANSKI et al. 2002).

$\mathrm{Ab}$ dem 3. Jh. kam es fast im gesamten Odergebiet zu einem Besiedlungsrückgang (DOMANSKI et al. 2002: Abb. 128). Eine Ausnahme bildete entlang des unteren Oderlaufs das Gebiet um den Krebssee und den Wustrowsee, das sogenannte Oderknie, das bereits früher, gegen Ende der Bronzezeit, gut besiedelt war.

Gegen Ende der Völkerwanderungszeit wird das Odergebiet durch Einwanderungen germanischer, slawischer und anderer ethnischer Gruppen aus der Peripherie des 
zerfallenden Römischen Reiches geprägt (VOß 1984). Gegen Ende des 5. Jh. hatte jedoch die Mehrzahl der west- und südwärts wandernden germanischen Stämme das Odergebiet verlassen.

\section{Mittelalter und Neuzeit}

Im Laufe des 6./7. Jh. - der genaue Zeitpunkt der Einwanderung der slawischen Stämme ist bisher nicht eindeutig belegt - erfolgte die Besiedlung durch slawische Stämme verschiedener Kulturen. Westlich des unteren Oderlaufs siedelten die Ucrani, das östliche Gebiet wurde vom Stamm der Prissani bevölkert, und die Leubuzzi nahmen das Gebiet um den mittleren Oderlauf ein (LECIEJEWICZ \& GRINGMUTH-DALLMER 2002: Abb. 130). Im 7./8. Jh. breitete sich im Untersuchungsgebiet die aus dem nördlichen Karpatenvorland stammende Feldberger Kultur aus (HENNING 1991).

In den Siedlungsräumen, in denen das Zusammentreffen unterschiedlicher Stämme zu sozialen Spannungen, Konflikten oder gar Kämpfen führte, entstanden zum Schutze der Einwohner mit Wällen umfriedete, befestigte Siedlungen oder Fluchtburgen. Die verschiedenen Stämme unterlagen einer rasanten Bevölkerungsentwicklung, so dass sich die Einwohnerzahlen vom 6. bis zum 10. Jh. vervierfachten, wie die Zunahme der Fundplätze (80 altslawische Fundplätze gegenüber 560 jungslawischen Fundplätzen) belegt.

Im 10. Jh. gründeten die Piasten das Königreich Polen. Sie herrschten bis zur Mitte des 14. Jahrhunderts. Die Stammeskönige und -fürsten ließen als Zeichen für Macht und Herrschaft sowie zum Schutz gegen Feinde und innere Unruhen Adels- und Fürstenburgen errichten. Nahe dem Krebssee und Wustrowsee ist eine Reihe slawischer Fürstenburgen bekannt (HERRMANN 1989).

Bevorzugte Siedlungsplätze waren zunächst die Terrassen und Anhöhen der Flusstäler sowie die Mündungsbereiche kleinerer Wasserläufe. Infolge der zunehmenden Bevölkerung und des ansteigenden wirtschaftlichen Wachstums stieg der Bedarf an Nutzflächen, so dass zur Schaffung neuer Siedlungsplätze sowie zur Deckung des enormen Holzbedarfs großflächige Rodungen einsetzten (LECIEJEWICZ \& GRINGMUTH-DALLMER 2002).

In der Landwirtschaft kam es infolge der Intensivierung der Ackerbaus zu einem Ausbau der Ackerflächen. Angebaut wurden vor allem Weizen, Roggen und Hirse. Die Bearbeitung der Äcker erfolgte weiterhin mit dem Hakenpflug. Die Produktion von Hülsen- und Ölfrüchten, darunter Saubohne, Linse, Flachs, Hanf und Mohn erfolgte in Gärten (NEEF 
2002). Neben dem Ackerbau spielte die Viehwirtschaft eine bedeutende Rolle. Sie konzentrierte sich vor allem auf die weniger zeitaufwendige Schweinehaltung, es wurden aber auch Rinder, Pferde, Ziegen und Schafe gehalten (BENECKE \& MAKOWIECKI 2002).

Von großer Bedeutung blieb die Verarbeitung von Eisen zur Herstellung von Arbeitsgeräten, Gegenständen des täglichen Bedarfs und Waffen. Im Laufe der Zeit entstanden, aufgrund der Spezialisierung des Handwerks und des Ausbaus der Tauschkontakte, vor allem im Bereich der Odermündung feste Handelsplätze, die sich teilweise zu Frühstädten weiterentwickelten (z.B. Wolin, Szczecin, Usedom). Untersuchungen der Kulturschichten solcher Frühstädte belegen die Verarbeitung verschiedener Werkstoffe wie Eisen und anderer Metalle, Geweih, Bernstein und Leder (LECIEJEWICZ \& GRINGMUTH-DALLMER 2002).

Seit der Mitte des 12. Jh., nach dem Tod des Königs Mieszo II., zerfiel die politische Staatsmacht - es entstanden mehrere pommersche Herzogtümer (HERRMANN 1989). Die Landesherren initiierten die Erschließung bisher unbesiedelter Räume, so dass ein großer Landesausbau erfolgte (GRINGMUTH-DALLMER 1995). Der Bedarf an Nutzungsflächen und Bauholz u.a. zur Errichtung von Gebäuden, Wehranlagen und Verkehrswegen war enorm, so dass große Waldflächen gerodet wurden. Die bereits bestehenden Frühstädte entwickelten sich schnell zu bedeutenden Handelszentren. In den gewässernahen Städten nahm der Fischfang eine bedeutende Rolle ein. Mit Hilfe von Salz, das vor allem im heutigen Kołobrzeg gewonnen wurde, wurde Fisch konserviert und nach Großpolen und Schlesien ausgeführt und gehandelt (LECIEJEWICZ \& GRINGMUTH-DALLMER 2002).

Im 13. Jh. kam es im Odergebiet durch die deutsche Ostkolonisation zu einer starken Wirtschaftsentwicklung und $\mathrm{zu}$ gravierenden Veränderungen in den Beziehungen zwischen Mensch und Umwelt. Durch den Zuzug der deutschen Siedler entstanden u. a. neue Siedlungsformen wie das planmäßig angelegte Straßendorf oder das Straßenangerdorf, welche sich erheblich von den allmählich gewachsenen Haufen- oder Rundplatzdörfern der slawischen Bevölkerung unterschieden (GRIESA 1998). Des weiteren kam es durch die Einführung des Beetpflugs mit Radvorgestell und durch die Einführung neuer Bodennutzungssysteme (Dreifelderwirtschaft) $\mathrm{zu}$ erheblichen Veränderungen in der Landwirtschaft. Aus einer ganzjährigen extensiven Weidewirtschaft auf den siedlungsnahen Wirtschaftsflächen entwickelte sich im Hochmittelalter eine gezielte Grünlandwirtschaft mit Wiesen und Weiden, die im Frühjahr und Herbst vom Vieh beweidet wurden und im Sommer zur Heugewinnung dienten (WILLERDING 2003). Durch den Bau von 
Wassermühlen, der das Aufstauen von Wasser erforderte, kam es zu gravierenden Änderungen der natürlichen Gewässersysteme (LECIEJEWICZ \& GRINGMUTH-DALLMER 2002).

$\mathrm{Zu}$ den im Laufe des 13. Jahrhunderts gegründeten Städten zählen u. a. Szczecin (1.243 n. Chr.) und Frankfurt/Oder (1.253 n. Chr.) (WINNIGE \& RoHLFS 1994). In den Städten bildeten die Handwerker, darunter z. B. Kürschner, Sattler, Schmiede, Leinenweber und Bäcker den größten Teil der Bevölkerung (RÜB 1998).

Zeugen des mittelalterlichen Aufschwungs in der Umgebung der Untersuchungsgebiete Krebssee und Wustrowsee sind das Augustiner-Kloster aus dem 13. Jh., die Marienkirche aus dem 14. Jh. und das Rathaus aus dem 15. Jh. in Chojna. In der Nähe des Pawski Ług, wurde in der zweiten Hälfte des 14. Jh. eine Ordensburg der Johanniter gebaut, die später, im 17./18. Jh., zum Schloss umgebaut wurde (HoROSZKO 1998).

Am Ende der deutschen Ostkolonisation hatte sich das bis in die Neuzeit bestehende Siedlungsbild in seinen Grundzügen herausgebildet (LECIEJEWICZ \& GRINGMUTHDALLMER 2002).

Das späte Mittelalter und die frühe Neuzeit waren jedoch nicht kontinuierlich durch einen ökonomischen Aufschwung gekennzeichnet. Pestepidemien (1348-1352, 1485-1497), der Dreißigjährige Krieg, Hochwasserkatastrophen (1665, 1736, 1785), Missernten und Hungersnöte führten mehrmals zu einem deutlichen Rückgang der Bevölkerung.

Im 18. Jh. veranlassten mehrere Hochwasserkatastrophen und Überschwemmungen die Bevölkerung zu umfangreichen Deichbauarbeiten an der Oder, der Warthe und an der Noteć (GRIESA 1998). Im gleichen Zeitrahmen erfolgten Maßnahmen zur Trockenlegung der Bruchlandschaften entlang der genannten Flüsse. Entwässerungswerke und Dämme schufen die Voraussetzungen für die ackerbauliche Nutzung mehrerer tausend Hektar humusreichen Bodens im Oderbruch, auf dem Weizen, Gerste und Raps angebaut wurden. Im Wartabruch entstanden vor allem große Flächen Weideland für die Viehwirtschaft. Auf den lehmigen Böden der Uckermark wurde u.a. Tabak kultiviert, während die sandigen Standorte der Oderniederung für Kartoffelpflanzungen gut geeignet waren. Der Nachteil der Begradigung des Oderlaufs und der Landgewinnung war ein Rückgang des Fischbestandes und infolge dessen ein Rückgang des traditionellen Fischergewerbes, das noch im 18. Jh. eine bedeutende Rolle für die Bevölkerung des Oderbruchs gespielt hatte (RüB 1998). 
Die agrarische Industrialisierung führte im 19. Jh. u. a. zum weiteren Ausbau der landwirtschaftlichen Nutzflächen. Der Anbau von Zuckerrüben war auf den fruchtbaren Böden der Oderniederung besonders ertragreich und gewinnbringend, so dass gegen Mitte des 19. Jh. allein im Oderbruch 18 Zuckerfabriken betrieben wurden. Die fortschreitende Industrialisierung hatte zur Folge, dass ein großer Teil der Landbevölkerung in die expandierenden Städte zog. Wegen des Waldreichtums in der Oderregion florierte besonders die Holz verarbeitende Industrie, darunter Dampfsägewerke, Werften und Möbelfabriken (RÜB 1998).

Verheerend waren die Folgen des zweiten Weltkrieges, die in ihren Dimensionen bei weitem die des ersten Weltkrieges und die des Dreißigjährigen Krieges übertrafen (GRIESA 1998). Viele Ortschaften im Odergebiet wurden durch Verwüstungen und Plünderungen zerstört. Nach Kriegsende führten die Bodenreform und die Bildung von landwirtschaftlichen Produktionsgenossenschaften westlich der Oder zu gravierenden Veränderungen in der Kulturlandschaft. Auf polnischer Seite setzte eine deutliche Urbanisierung ein, die viele Kleinstädte mit 10.000-20.000 Einwohnern hervorbrachte (GRIESA 1998).

Zum partiellen Schutz der heute überwiegend durch Ackerbau geprägten Landschaft wurden mehrere Areale als Naturschutzgebiete ausgewiesen. Seit 1995 besteht zwischen Hohensaaten/Cedynia und Mescherin/Gryfino der deutsch-polnische Nationalpark „Unteres Odertal“. Weiter nördlich schließt der Wolinski Park Narodowy (Woliner Nationalpark) an. Im Einzugsbereich der Warta besteht der Drawienski Park Narodowe (Drauer Nationalpark). Der Mündungsbereich der Warta ist bekannt als eines der größten Vogelschutzgebiete Europas. Zu den Landschaftsparks, die ebenfalls dem gesetzlichen Naturschutz unterstehen, zählen u. a. der Cedynski (Zehdener) und der Łagówski Park Krajobrazowy (Lagower Landschaftspark) (LINKOwSKI 1998). 


\section{Material und Methoden}

\subsection{Laborarbeiten}

Nach der Sedimentansprache wurden die Kerne photographiert und beprobt. Dabei wurden im Abstand von $8 \mathrm{~cm}$ mit Hilfe von Einwegspritzen etwa 1 bis $2 \mathrm{ml}$ Sediment entnommen, in Folie verpackt und bis zur Aufbereitung im Kühlschrank aufbewahrt. Die Pollenaufbereitung erfolgte im wesentlichen nach den in BEUG (1957) beschriebenen Acetolyseverfahren. Zur Bestimmung der Pollenkonzentration wurde den Proben vor der Aufbereitung eine definierte Menge Lycopodium-Sporen zugesetzt (STOCKMARR 1971). Sehr sandige Proben wurden zur Entfernung der mineralischen Bestandteile einer etwa 24stündigen HF-Behandlung unterzogen. Schließlich wurde mit Hilfe eines Ultraschallbades das Feinmaterial $(<10 \mu \mathrm{m})$ aus den Pollenproben herausgesiebt. Zur Aufbewahrung wurden die Proben dann mit Glycerin in Gläschen überführt.

An dieser Stelle soll eine kurze Methodenkritik angeführt werden. Grund dafür liefert das Pollendiagramm von Rybojady, dessen Gehölzpollenkurven, insbesondere die PinusKurve, sehr starken Schwankungen unterliegen. Dies findet seine Erklärung wahrscheinlich in der Probenaufbereitung, die in unterschiedlich ausgestatteten Laboren und zum Teil auch durch verschiedene Personen erfolgte. Außerdem fand die Aufbereitung der Sedimentproben in zwei Etappen statt: Zunächst wurden die in einem Abstand von $16 \mathrm{~cm}$ entnommenen Sedimentproben in einem Labor aufbereitet, anschließend erfolgte nach Verdichtung des Probenabstandes die Aufbereitung der daraus resultierenden Sedimentproben in einem anderen Labor. Die Anwendung verschiedener Zentrifugen mit ungleichen Drehzahlen und Beschleunigungen führte möglicherweise $\mathrm{zu}$ einer unterschiedlichen Anreicherung von Pinus-Pollenkörnern im Pellet. Andererseits könnte eine zu lange Ultraschallbehandlung zur Zerstörung von Pinus-Pollenkörnern bzw. zur Abtrennung der Luftsäcke geführt haben, was bei der anschließenden Auszählung zu Ungenauigkeiten geführt haben kann. Hier müsste eine Überprüfung erfolgen, in dem Sedimentproben des gleichen Ausgangsmaterials in beiden Laboren aufbereitet und anschließend nochmals analysiert werden.

\subsection{Pollenanalyse}

Die Pollenproben wurden überwiegend bei 500-facher Vergrößerung an einem Zeiss- oder einem Leica-Mikroskop auf mindestens 500 Baumpollen (BP) ausgezählt. Für die 
Bestimmung der Getreide-Typen sowie für die Identifizierung schwierig bestimmbarer Pollenkörner (PK) wurden eine 1250fache Vergrößerung und teilweise ein Phasenkontrast eingesetzt. Die Größe der Getreide-PK und der Pollenkörner vom Humulus/Cannabis-Typ wurde zum überwiegenden Teil notiert, um später eine genauere Differenzierung nach BEUG (1961) und DÖRFLER (1989) vornehmen zu können.

Als Bestimmungshilfen standen die Vergleichssammlungen und Photokarteien der Abteilung Palynologie und Quartärwissenschaften in Göttingen, sowie des Referats Naturwissenschaften des Deutschen Archäologischen Institutes in Berlin zur Verfügung.

Zusätzlich wurde folgende Bestimmungsliteratur verwendet:

Beug (1961), FAEGRI et al. (1989), MoORE et al. (1991), KoMAREK \& FotT (1983), PUnT \& Clarke (1976; 1980; 1981; 1984; 1991; 1995), WAGENITZ (1955). Die Bezeichnung der Pollentypen folgt den Angaben in FAEgri et al. (1989) und Moore et al. (1991); die Nomenklatur der höheren Pflanzen richtet sich nach OBERDORFER (1994).

\subsection{Darstellung der Ergebnisse}

Die Ergebnisse der Pollenanalysen sind als Prozentdiagramme dargestellt. Die Bezugssumme bildet die Summe des Gehölzpollens ohne Corylus.

Es wurden folgende Gruppen gebildet:

- Baumpollen (BP): alle Gehölzpollentypen exclusive Corylus

- Eichenmischwald (EMW): Quercus, Ulmus, Fraxinus, Tilia platyphyllos, Tilia cordata, Acer

- Nichtbaumpollen (NBP): alle terrestrischen Kräuter

- Kulturpflanzen: Hordeum-Typ, Triticum-Typ, Secale cereale, Avena-Typ, Getreide indet., Fagopyrum, Zea mays

- Sekundäre Siedlungszeiger: Cannabis/Humulus-Typ, Brassicaceae, Centaurea cyanusTyp, Chenopodiaceae, Convolvulus arvensis, Fabaceae, Plantago lanceolata-Typ, Plantago major/media-Typ, Rumex acetosalacetosella-Typ, Polygonum aviculare, Polygonum persicaria-Typ, Scleranthus, Trifolium-Typ, Urtica, Vicia-Typ, Xanthium

Für die Auswertung und Interpretation der pollenanalytischen Befunde wurden die Pollendiagramme in lokale Pollenzonen (local pollen assemblage zone: L PAZ) unterteilt. 
Diese Einteilung richtet sich in erster Linie nach visuellen Gesichtspunkten. Anschließend erfolgte eine Zuordnung der lokalen Pollenzonen zu den von FIRBAS (1949) ermittelten waldgeschichtlichen Abschnitten der postglazialen Vegetationsentwicklung. Die auf BLYTT (1881), SERNANDER (1908), JESSEN (1949) und IVERSEN (1954) zurückgehenden klimatographischen Bezeichnungen wurden von FIRBAS $(1949 ; 1952)$ als Benennung der waldgeschichtlichen Abschnitte übernommen. In der vorliegenden Arbeit werden diese ursprünglich klimatographisch gemeinten Bezeichnungen in Anlehnung an FIRBAS (1949) und FIRBAS (1952) im rein biostratigraphischen Sinne verwendet.

\subsection{Sedimentstratigraphie vom Wustrowsee und Krebssee}

Im September 1999 wurden von einer schwimmenden Plattform aus bei einer Wassertiefe von 14,2 $\mathrm{m}$ beim Krebssee und 9,7 $\mathrm{m}$ beim Wustrowsee mit Hilfe eines UsingerBohrgerätes mehrere Bohrkerne $(1=2 \mathrm{~m}, \emptyset=8 \mathrm{~cm})$ erbohrt. Aus dem Krebssee konnte auf diese Weise ein insgesamt $4 \mathrm{~m}$ langes Profil (KBS2) und aus dem Wustrowsee ein 14,5 m langes Profil (WUS3 und WUS1) erbohrt werden. Zur Überbrückung möglicher Sedimentlücken wurden jeweils Parallelbohrungen mit einer Überlappung von $50 \mathrm{~cm}$ durchgeführt. Die Bohrkerne wurden an Land mit Hilfe einer speziellen Vorrichtung in PVC-Halbrohre ausgedrückt und bis zur Sedimentansprache und Probenentnahme in Folie verpackt und kühl gelagert. Die Tabelle 1 und Tabelle 2 stellen die Sedimentstratigraphie der Profile Wustrowsee und Krebssee dar.

\subsection{Sedimentstratigraphie von Rybojady und Pawski Ług}

Die Profile Rybojady (R1.00) und Pawski Ług (L1.00) wurden im Oktober 2000 mit Hilfe eines sogenannten Russischen Kammerbohrers von der heutigen Mooroberfläche beginnend erbohrt. Das Profil Rybojady wurde mit einer Bohrkammer, die eine Länge von $50 \mathrm{~cm}$ aufweist, erbohrt. Es erfolgte eine Parallelbohrung mit einer Überlappung von $20 \mathrm{~cm}$.

Das Profil Pawski Ług wurde mit einer 100-cm-Bohrkammer erbohrt. Die Parallelbohrung wurde ohne Überlappung niedergebracht. Die Verpackung und Aufbewahrung erfolgte wie bereits oben (Kap. 4.4) beschrieben.

Da die jüngsten Ablagerungen zum überwiegenden Teil aus grober, unzersetzter organischer Substanz (Sphagnum- und Vacciniumreste) bestehen, war es nicht möglich, mit dem Kammerbohrer aus diesem Bereich ein zusammenhängendes Profil zu erbohren. 
Die unzersetzten Sphagnum- und Vacciniumästchen ließen sich mit dem Bohrgerät nicht zerschneiden, sondern wurden verdrängt bzw. kompaktiert. Um diesen Bereich dennoch bearbeiten zu können, wurde im Januar und Juni 2003 im Rybojady-Moor mit Hilfe eines Spatens ein Kurzprofil gegraben (R1.03). Dieses schließt oben an das bereits bestehende Profil (R1.00) an. Im Pawski Ług konnte ein entsprechendes Profil leider nicht gegraben werden. Die Tabelle 3 und Tabelle 4 stellen die Sedimentstratigraphie der Profile Rybojady und Pawski Ług dar.

\subsection{Radiocarbondatierungen}

Die insgesamt siebenundzwanzig Radiocarbondatierungen wurden im Leibniz-Labor für Altersbestimmung und Isotopenforschung der Universität Kiel mittels der AMS-Technik (accelerator mass spectrometry-Technik) durchgeführt. Alle Daten wurden mit dem Radiocarbon Calibration Program „CALIB rev 4.3“ (Datensatz 2) (STUIVER et al. 1998) in Kalenderjahre umgewandelt.

Den Datierungen lagen Pollensuspensionen zugrunde, deren Aufbereitung nach einem speziellen Verfahren (BROwN et al. 1992) im Pollenlabor des Deutschen Archäologischen Instituts in Berlin erfolgte. Die Ergebnisse der Radiocarbondatierungen sind in Tabelle 5 aufgeführt.

\subsection{Zeit-Tiefen-Diagramme}

Den Zeit-Tiefen-Diagrammen ist die Sedimentzuwachsrate $\mathrm{zu}$ entnehmen. Je mehr Datierungen aus einem Profil vorliegen, desto wahrscheinlicher ist der Verlauf der durch Interpolation ermittelten Sedimentationskurve. Aus den Veränderungen der Sedimentationskurve, deren Steigung der Sedimentationsrate (cm/a) entspricht, lassen sich u. a. Rückschlüsse auf die Sedimentationsbedingungen ziehen.

\subsubsection{Wustrowsee}

Die Sedimentationsrate stieg ab ca. 9.400 cal. B.C. von zuvor $0,07 \mathrm{~cm}$ auf $0,13 \mathrm{~cm}$ pro Jahr an (Abbildung 1). Bis ca. $3.500 \mathrm{cal}$. B.C. blieben die Sedimentationsbedingungen relativ konstant. Darauf folgte ein Anstieg der Sedimentationsrate auf 0,25 bzw. 0,28 cm pro Jahr (W 5a, W 5b). Ab ca. 2.800 cal. B.C. bis ca. 400 cal. B.C. betrug die Sedimentzuwachsrate etwa 0,2 cm pro Jahr. Danach stieg sie erneut leicht an. Ab ca. 120 cal. B.C. betrug die Zuwachsrate bis ca. $660 \mathrm{cal}$. A. D. nur noch 0,02 cm pro Jahr. 


\subsubsection{Rybojady}

Das Zeit-Tiefen-Diagramm von Rybojady (Abbildung 2) zeigt einen deutlichen Anstieg der Sedimentationsrate ab 7.000 cal. B.C. und zwischen $1.690 \mathrm{cal}$. B.C. und $1.280 \mathrm{cal}$. B.C. $(312-428 \mathrm{~cm})$. Hinweise auf einen Sedimentwechsel sind der Sedimentstratigraphie nicht zu entnehmen. Allerdings korreliert das Ansteigen der Sedimentationsrate mit einer Zunahme der Siedlungsaktivitäten.

\subsubsection{Pawski Ług und Krebssee}

Da von den Profilen vom Pawski Ług und Krebssee nur drei bzw. zwei Datierungen vorliegen und diese einen großen Abstand voneinander aufweisen, lassen sich keine Aussagen hinsichtlich sich ändernder Sedimentationsbedingungen ableiten. Der Vollständigkeit halber werden die Zeit-Tiefen-Diagramme dieser Profile trotzdem abgebildet (Abbildung 3 und Abbildung 4). 


\section{Diagrammbeschreibung}

\subsection{Wustrowsee}

Das Pollendiagramm des Wustrowsees basiert auf der pollenanalytischen Bearbeitung zweier Profile: WUS 1 und WUS 3. Der Kern WUS 1 umfasst den Zeitraum vom Älteren Atlantikum bis ins Jüngere Subatlantikum, der Kern WUS 3 beginnt mit dem Präboreal und endet mit dem Subboreal. Da die Sedimente keine deutliche Schichtung aufweisen, war eine makroskopische Parallelisierung der Kerne nicht möglich. Die Überlappung der beiden Profile wurde daher pollenanalytisch abgesichert. Demzufolge erfolgt der Kernwechsel von WUS 1 nach WUS 3 bei $1233 \mathrm{~cm}$ Tiefe.

Im Folgenden werden die wesentlichen Vegetationsentwicklungen der einzelnen lokalen Pollenzonen (L PAZ) beschrieben. In der Tabelle 6 sind die verschiedenen lokalen Pollenzonen des Pollendiagramms vom Wustrowsee, deren chronologische Einordnung und Zuordnung zu archäologischen Kulturstufen als Übersicht zusammengestellt.

\section{W 1 (1554-1438 cm): Betula-Ulmus-PAZ}

Den Hauptbestandteil des Gehölzpollens bildet Betula-Pollen mit Werten bis zu $90 \%$. Daneben sind Pinus, Juniperus und Salix mit geringen Werten vertreten. Ab $1505 \mathrm{~cm}$ liegen die Ulmus- und Corylus-Kurven als geschlossene Kurven vor. Alnus und Quercus treten sporadisch auf. Der Anteil des NBP, der sich überwiegend aus Poaceae, Artemisia, Chenopodiaceae, Filipendula und Cyperaceae zusammensetzt, sinkt von anfangs 15,7\% auf 4,9 \% . Unter den Grünalgen ist Botryococcus mit erhöhten Werten (bis $10 \%$ ) präsent.

Die Pollenkonzentration beträgt zu Beginn des Abschnitts ca. 90.000 PK pro ml Sediment. Unter Schwankungen steigt die Pollenkonzentrationskurve bis zum Ende des Abschitts auf Werte um 800.000 PK pro ml Sediment an.

Die Grenzziehung zwischen W 1 und W 2 erfolgte anhand des steilen Anstiegs der Corylus-Kurve von $14,6 \%$ auf $45,5 \%$.

\section{W 2 (1438 - 1317 cm): Pinus-Corylus-Quercus-PAZ}

Das Baumpollenspektrum dieser PAZ wird beherrscht durch den kontinuierlichen Anstieg von Corylus bis zu einem Maximum $230 \%$. Danach fallen die Werte auf ca. $100 \%$ zurück. Die Ulmus-Kurve zeigt bei insgesamt geringeren Werten einen parallelen Verlauf (Maxímum 12,3\%). Der Anteil von Betula, die bislang vorherrschend war, erreicht nur 
noch $35 \%$. Die durchschnittlichen Pinus-Werte liegen nun bei ca. $30 \%$. Ab $1337 \mathrm{~cm}$ kommt Quercus mit geschlossener Pollenkurve vor, ab $1401 \mathrm{~cm}$ steigt die Quercus-Kurve steil an auf 12,5\%. Tilia und Fraxinus sind mit geringen Werten unregelmäßig vertreten.

Das Spektrum des NBP zeigt bis auf die Zunahme von Poaceae (bis 6,6 \%), Calluna (bis $1,7 \%$ ) und Filicinae (bis 4,5\%) keine wesentlichen Veränderungen.

Unter den Grünalgen kommt es zu einem Rückgang von Botryococcus und zu einer leichten Zunahme von Pediastrum boryanum.

Die durchschnittliche Pollenkonzentration zeigt einen deutlichen Rückgang von zuvor 800.000 PK pro ml auf ca. 300.000 PK pro ml.

Der Übergang zur nächsten L PAZ wird durch den steilen Anstieg der Alnus-Kurve und dem Rückgang von Corylus gekennzeichnet.

\section{W 3 (1317-1097 cm): Alnus-Tilia-PAZ}

Der steile Anstieg der Alnus-Kurve von 4,3\% auf ca. $30 \%$ und der Anstieg der TiliaWerte kennzeichnen diese PAZ. Corylus sinkt von zuvor $100 \%$ kontinuierlich auf ca. $30 \%$, die Betula-Kurve pendelt nach einem erneuten Rückgang bei Werten um $25 \%$. Die Pinus-Kurve verhält sich gegenläufig zur Betula-Kurve, steigt bis zur Abschnittsmitte bis $\mathrm{zu} 40 \%$ an und fällt danach auf Werte unter $10 \%$ zurück. Unter den EMW-Arten steigt Quercus kontinuierlich bis auf 28,2\% an, Ulmus pendelt zwischen 8-10\%. Bis $1441 \mathrm{~cm}$ schwankt die Tilia-Kurve um $3 \%$, danach liegen leicht erhöhte Werte um $7 \%$ vor. Fraxinus ist wie zuvor unregelmäßig vertreten. Ebenfalls unregelmäßig sind seit Beginn dieses Abschnitts Hedera, Viscum und Picea präsent. Zum ersten Mal wurden einzelne Pollenkörner von Fagus und Carpinus gefunden.

Die Anteile des NBP zeigen bis auf einen leichten Anstieg von Artemisia ab $1173 \mathrm{~cm}$ keine wesentlichen Veränderungen.

$\mathrm{Ab}$ etwa $1229 \mathrm{~cm}$ treten Sporen von zuvor vermehrt vorkommenden Filicinae nur noch sporadisch auf.

Das Grünalgenspektrum zeigt keine signifikanten Veränderungen. 
Die mittlere Pollenkonzentration pendelt nach einem Rückgang (ca. 200.000 PK pro ml) um 350.000 PK pro ml Sediment.

Die Grenze zum nächsten PAZ orientiert sich am Verlauf der Fraxinus-Kurve.

\section{W 4 (1097-934 cm): EMW-Fraxinus-PAZ}

Das Einsetzen der geschlossenen Fraxinus-Kurve kennzeichnet den Beginn dieser PAZ, die im wesentlichen durch eine starke Repräsentanz von EMW-Arten gekennzeichnet ist. Während die Pinus-Kurve unter Schwankungen von $10 \%$ auf $30 \%$ ansteigt, sinkt diejenige von Betula ebenfalls unter Schwankungen bis zu ca. $15 \%$. Ein leichter Rückgang zeigt sich auch bei Ulmus (von $10 \%$ auf 7,1\%). Die Tilia-Werte steigen bis zur Abschnittsmitte gleichmäßig auf 6,1\% an und fallen ab $963 \mathrm{~cm}$ auf 2,9\% zurück. Die Fraxinus-Kurve pendelt zwischen $0,3 \%$ bis 2,3\%. Ab $1027 \mathrm{~cm}$ tritt erstmals Acer mit geringen Werten auf. Die Anteile von Alnus bewegen sich im Schnitt um $20 \%$.

Bei den krautigen Arten sind keine signifikanten Kurvenanstiege zu verzeichnen. Die anfänglich leicht erhöhten Artemisia-Werte nehmen ab $1043 \mathrm{~cm}$ wieder ab. Gegen Ende der PAZ (971 cm) tritt zum ersten Mal Plantago lanceolata auf.

Die Anteile der Farnsporen sind erneut leicht angestiegen, darunter Pteridium, Polypodium und Filicinae.

Grünalgen sind nur noch mit sehr geringen Anteilen vertreten.

Die Pollenkonzentration ist kaum verändert und erreicht im Mittel ca. 320.000 PK pro ml Sediment.

Der Übergang zur nächsten PAZ wird durch den Beginn der geschlossenen Fagus-Kurve gekennzeichnet.

\section{W 5 a (934-734 cm): EMW-Fagus-Plantago lanceolata-PAZ}

Das Baumpollenspektrum wird trotz des Anstiegs von Pinus und Alnus (bis $38 \%$ ) durch EMW-Arten beherrscht. Nach dem gleichmäßigen Anstieg von Pinus auf ca. $51 \%$ fallen die Werte ab $787 \mathrm{~cm}$ unter Schwankungen auf $21 \%$ zurück. Gleichzeitig steigt die BetulaKurve ab $787 \mathrm{~cm}$ leicht an. Unter den thermophilen Arten zeigt sich zu Beginn der PAZ mit dem Rückgang von Ulmus von 7,1\% auf 3,8 \% eine deutliche Veränderung. Diesem Rückgang folgt ab $787 \mathrm{~cm}$ ein erneuter Anstieg auf 6,3\%, gefolgt von einem wiederholten Rückgang auf 2,2\% gegen Ende der PAZ. Parallel zu dem erneuten Ulmus-Anstieg steigen auch die Quercus- und Tilia-Werte an, während die Fraxinus-Werte unverändert 
zwischen $0,3 \%$ und $1,2 \%$ pendeln. Hedera ist wie zuvor noch immer regelmäßig vertreten, Viscum dagegen kommt nur noch ein Mal vor und ist in allen folgenden Diagrammabschnitten ebenfalls nur sporadisch vorhanden. Während Fagus mit dem Beginn der PAZ die empirische Pollengrenze und am Ende der PAZ Werte um etwa $1 \%$ erreicht, ist Carpinus mit Werten bis 0,6 \% noch immer lückenhaft vertreten.

Die Anteile des NBP sind leicht angestiegen, bedingt durch das vermehrte Auftreten von Rumex und Plantago lanceolata. Letzterer hat ab $907 \mathrm{~cm}$ eine geschlossene Pollenkurve.

Unter den Farnsporen zeigt sich ein Rückgang von Pteridium.

Ab $843 \mathrm{~cm}$ steigt der Anteil der Grünalgen, bedingt durch die Zunahme von Botryococcus und Pediastrum boryanum, wieder an.

Mit ca. 290.000 PK pro ml Sediment ist die durchschnittliche Pollenkonzentration in diesem Abschnitt etwas geringer als in der vorherigen PAZ.

Die Abgrenzung zur nächsten PAZ erfolgte anhand der Corylus- und Tilia-Kurve, die an dieser Stelle deutlich abfallen.

\section{W 5b (734-599 cm): Carpinus-Cerealia-NBP-PAZ}

Das Gehölzpollenspektrum wird nun von Alnus dominiert. Nach Erreichen des Maximums $(48,9 \%)$ bei $691 \mathrm{~cm}$, fällt die Alnus-Kurve bis auf $25 \%$ zurück. Die Anteile von Pinus und Betula pendeln zunächst um $15 \%$, letztere steigen gegen Ende des Abschnitts leicht an $(21,5 \%)$. Corylus liegt mit schwankenden Anteilen zwischen 13,4 und $30 \%$ vor. Unter den EMW-Arten zeigt sich ein rückläufiger Trend, bedingt durch den deutlichen Rückgang von Quercus (34,9\% auf 18,2\%) und Tilia (2,5\% auf $1 \%)$. Fagus ist mit Werten zwischen $0,7 \%$ bis 2,1\% kontinuierlich vertreten, während Carpinus erst ab $699 \mathrm{~cm}$ mit geschlossener Kurve und Werten zwischen 0,7 \% und 1,7 \% vorliegt.

Die Anteile des NBP sind mit Maximalwerten von 15,4\% erstmals deutlich erhöht. Bedingt wird dieser Anstieg durch die Zunahme der Poaceae von zuvor knapp $2 \%$ auf $7 \%$ sowie durch die Zunahme von Chenopodiaceae (bis 2,0\%), Rumex (bis 2,2\%) und Plantago lanceolata (bis 3,1\%). Pollen vom Getreide indet.-Typ und erstmals auch vom Triticum-Typ wurde nur sehr selten gefunden.

Farnsporen sind noch seltener als zuvor.

Unter den Grünalgen tritt Pediastrum zwischen $699 \mathrm{~cm}$ und $675 \mathrm{~cm}$ mit deutlich erhöhten Werten (um $12 \%$ ) hervor. 
Die abnehmende Tendenz der Pollenkonzentration setzt sich weiter fort. In dieser PAZ liegt sie durchschnittlich nur noch bei ca. 235.000 PK pro ml Sediment.

Der Rückgang des NBP und der Anstieg der Quercus-Kurve kennzeichnen den Übergang zur folgenden PAZ.

\section{W 5c (599-527 cm): EMW-Fagus-Carpinus-PAZ}

Dieser Abschnitt ist durch einen Wiederanstieg der Quercus-Werte und einen Rückgang des NBP-Anteils gekennzeichnet. Betula und Corylus verhalten sich nahezu unverändert, Pinus fällt gegen Ende des Abschnitts auf geringe Werte um $7 \%$ zurück. Unter den EMW-Arten kommt es mit Ausnahme der erhöhten Quercus-Werte zu keinen nennenswerten Veränderungen. Die Alnus-Werte steigen wieder leicht an und erreichen am Ende ca. $30 \%$. Während Fagus weiterhin zwischen 1,2\% und 2,2\% schwankt, liegen die Carpinus-Werte in der zweiten Hälfte des Abschnitts durchgehend über $2 \%$.

Dem deutlichen Anstieg des NBP des vorherigen Abschnitts folgt nun ein Rückgang, vor allem bedingt durch den Rückgang von Poaceae, Artemisia, Chenopodiaceae, Plantago lanceolata und Rumex. Der Nachweis von Getreide beschränkt sich auf das Vorkommen eines Pollenkorns vom Hordeum-Typ.

Unter den Farnsporen tritt Polypodium vermehrt auf.

Ein kontinuierlicher Anstieg ist bei der Grünalge Pediastrum boryanum zu verzeichnen.

Die durchschnittliche Pollenkonzentration zeigt sich unverändert.

Die Abgrenzung zur nächsten PAZ erfolgte anhand des Wiederanstiegs des NBP und dem Rückgang von Quercus.

\section{W 5d (527-399 cm): Fagus-Carpinus-NBP-PAZ}

Die gleichmäßige Zunahme von Fagus (bis 4,8\%) und Carpinus (bis 5,8\%) und ein Anstieg des NBP (bis 15,9\%) sind kennzeichnend für diese PAZ. Eine leicht zunehmende Tendenz zeigen Pinus, Betula und Alnus, während bei Corylus und den EMW-Arten ein Rückgang zu verzeichnen ist. Corylus fällt von $20 \%$ auf $10 \%$, Ulmus fällt von zuvor $2 \%$ auf ca. $1 \%$ zurück. Die Anteile von Tilia liegen ab $483 \mathrm{~cm}$ unter $1 \%$.

Die Zunahme des NBP wird bedingt durch den Anstieg von Poaceae (bis 7,0\%), Artemisia (bis 4,0\%), Chenopodiaceae (bis 0,5\%), Rumex (bis 1,8\%) und Plantago 
lanceolata (bis 2,5\%). Getreidepollen kommt selten vor und beschränkt sich auf Einzelfunde. Leicht erhöht sind die Anteile der Cyperaceae.

Unter den Farnsporen kommt Polypodium kaum noch vor, die Anteile der Filicinae haben dagegen leicht zugenommen.

$\mathrm{Ab} 443 \mathrm{~cm}$ fallen die bis dahin erhöhten Anteile von Pediastrum boryanum (bis $10 \%$ ) auf Werte $<1 \%$ zurück.

Die durchschnittliche Pollenkonzentration ist ein wenig gesunken und beträgt jetzt etwa 200.000 PK pro ml Sediment.

Die Grenze zur folgenden PAZ wird durch den Rückgang der Corylus-Werte unter $10 \%$ definiert.

\section{W 6a (399-343 cm): Pinus-EMW-PAZ}

Zunehmende Pinus-Anteile (bis $50 \%$ ) und ein Rückgang von Corylus < $10 \%$ zählen zu den wesentlichen Veränderungen des Baumpollenspektrums dieser PAZ.

Nach einem Rückgang der Betula-Werte erfolgt ein erneuter Anstieg auf ca. $32 \%$. Quercus geht zunächst von zuvor 18,9\% auf 12,2\% zurück und steigt am Ende wieder bis zu $23 \%$ an. Ulmus erreicht nur noch maximal 1,2\%, Tilia und Fraxinus liegen im Mittel bei 0,4\%. Mit Ausnahme eines Gipfels von $36 \%$ pendeln die Anteile von Alnus um $23 \%$. Die Carpinus-Werte fallen nach einem Anstieg (5,4\%) wieder auf 2,5\% zurück, diejenigen von Fagus pendeln zwischen 1,8\% und 4,3\%.

Der Anteil des NBP steigt nach einem Rückgang auf $5 \%$ wieder bis zu ca. $10 \%$ an. Hervorgerufen wird dies vor allem durch das Verhalten von Poaceae, Plantago lanceolata, Rumex und Calluna.

Farnsporen treten nur noch sporadisch auf.

Die Grünalgen Botryococcus und Pediastrum boryanum sind erneut mit leicht zunehmenden Werten präsent.

Mit durchschnittlich 220.000 PK pro ml Sediment hat die Pollenkonzentration leicht zugenommen.

Die Abgrenzung zum nächsten Diagrammabschnitt erfolgte anhand der abnehmenden NBP-Werte. 


\section{W 6b (343-304 cm): Pinus-Betula-Quercus-PAZ}

Dieser Diagrammabschnitt ist durch einen markanten Rückgang der NBP-Kurve von zuvor $10 \%$ auf $2 \%$ bis $3 \%$ gekennzeichnet.

Die Pinus-Werte halten ein Niveau von ca. $20 \%$, Betula fällt auf $24 \%$ zurück. Corylus und die EMW-Arten weisen keine wesentlichen Veränderungen auf. Die Anteile von Alnus, Fagus und Carpinus zeigen eine zunehmende Tendenz. Fagus erreicht am Ende $5,5 \%$, Carpinus ist mit 5,7\% vertreten.

Die Anteile von Poaceae (bis 3,4\%), Artemisia (bis 0,6\%), Chenopodiaceae (bis 0,2 \%), Plantago lanceolata (bis 0,3\%) und Rumex (bis 0,8\%) sind so gering, dass der Anteil des NBP mit $2 \%$ bis $6 \%$ die bislang niedrigsten Werte aufweist.

Grünalgen sind nur in geringer Anzahl vertreten.

In dieser PAZ steigt die durchschnittliche Pollenkonzentration vorübergehend auf 360.000 PK pro ml Sediment an.

Die Grenze zum nächsten Diagrammabschnitt ist durch den markanten Rückgang von Pinus und den erneuten Anstieg des NBP gekennzeichnet.

\section{W 6c (304-214 cm): Fagus-Carpinus-NBP-PAZ}

Das Baumpollenspektrum ist gekennzeichnet durch den Rückgang von Pinus von ca. $25 \%$ auf Werte um $3 \%$ sowie durch einen deutlichen Anstieg von Fagus auf $14 \%$. Betula, Quercus und Alnus weisen nach einem anfänglichen Anstieg eine abnehmende Tendenz auf. Tilia und Fraxinus sind bis zum Diagrammende nur noch mit geringen Werten um 0,4\% bzw. 0,2\% vertreten, Ulmus pendelt zwischen 0,4\% und 1,6\%. Viscum ist noch ein Mal vertreten und kommt dann bis zum Diagrammende nicht mehr vor, während Hedera weiter mit Einzelfunden belegt ist.

Bis $251 \mathrm{~cm}$ sind die Anteile des NBP mit Werten um $7 \%$ leicht erhöht, danach pendeln sie um 4,5\%. Hervorgerufen wird der Rückgang durch die Abnahme von Poaceae, Artemisia, Rumex und Plantago lanceolata. Ab $251 \mathrm{~cm}$ tritt erstmals Secale auf. Daneben ist Pollen vom Getreide-Typ, vom Triticum-Typ und vom Hordeum-Typ mit geringen Werten präsent.

Die Anteile von Botryococcus sind während der gesamten PAZ erhöht (um $10 \%$ ), diejenigen von Pediastrum boryanum zeigen zwischen $267 \mathrm{~cm}$ und $251 \mathrm{~cm}$ ein Maximum von ca. $13 \%$. 
Die durchschnittliche Pollenkonzentration hat wieder abgenommen und liegt nun bei ca. 210.000 PK pro ml Sediment.

Die Abgrenzung zur nächsten PAZ erfolgte anhand des Anstiegs von Fagus und Carpinus und anhand des NBP-Rückgangs.

\section{W 6d (214-183 cm): Fagus-Carpinus-PAZ}

Diese PAZ wird durch das Erreichen maximaler Fagus- und Carpinus-Anteile charakterisiert.

Fagus erreicht maximal 18,8\%, Carpinus erreicht ein Maximum von 12,0\%. Die Anteile von Pinus betragen noch ca. $10 \%$, Betula ist mit $20 \%$ vertreten, Quercus ist mit Anteilen um $15 \%$ und Alnus mit Anteilen um $25 \%$ vertreten.

Auffällig sind die geringen Anteile des NBP (2,4\%), verursacht durch den Rückgang von Poaceae, Artemisia, Rumex und Plantago lanceolata. Getreidepollen fehlt in diesem Abschnitt völlig.

Auch die Anteile der Algen zeigen eine deutlich rückläufige Tendenz.

Die durchschnittliche Pollenkonzentration hat sich nicht signifikant verändert.

Der Übergang zum nächsten Diagrammabschnitt wird durch die abfallenden Kurven von Fagus und Carpinus sowie durch den Anstieg der NBP-Kurve charakterisiert.

\section{W 6e (183-111 cm): Quercus-Betula-NBP-PAZ}

Dieser Abschnitt ist durch den kontinuierlichen Rückgang von Fagus (4,1\%) und Carpinus $(2,8 \%)$ und den gleichzeitigen Wiederanstieg von Pinus (bis $20 \%$ ), Betula (bis $34 \%$ ) und des NBP (bis 17,9\%) gekennzeichnet.

Bedingt wird der Anstieg des NBP vor allem durch die erneute Zunahme von Poaceae (bis $6 \%$ ), Artemisia (bis 2,5\%), Rumex (bis 2,9\%) und Plantago lanceolata. Unter den Getreide-Typen sind neben Secale der Triticum-Typ und der Getreide indet.-Typ wieder häufiger vertreten. Erstmals wurde ein Pollenkorn vom Avena-Typ gefunden. Des weiteren steigt die Anzahl der NBP-Taxa allmählich an.

Botryococcus und Pediastrum boryanum sind wieder häufiger nachgewiesen.

Die Pollenkonzentration schwankt zwischen 115.000 und 300.000 PK pro ml und zeigt eine leicht zunehmende Tendenz. 
Anhand der abfallenden Kurven von Betula, Alnus und des NBP und anhand des Anstiegs der Quercus-Werte wird diese PAZ von der folgenden abgegrenzt.

\section{W $6 f(111-87 \mathrm{~cm})$ : Quercus-Betula-PAZ}

Dieser Diagrammabschnitt ist durch einen erneuten Rückgang von Pinus (9\%), Betula (25\%) und Alnus (25\%) charakterisiert. Die Anteile von Fagus und Carpinus pendeln um $7 \%$ bzw. $4 \%$, diejenigen von Quercus steigen von zuvor $15 \%$ auf ca. $30 \%$ an.

Die Kurve des NBP zeigt einen deutlichen Einschnitt, verursacht durch den wiederholten Rückgang von Poaceae, Artemisia, Rumex und Plantago lanceolata. Getreide ist durch geringe, aber regelmäßige Funde von Secale mit Werten bis $0,5 \%$ und vereinzelten Nachweisen vom Hordeum-Typ belegt.

Das Spektrum der weiteren Taxa zeigt keine signifikanten Veränderungen.

Die durchschnittliche Pollenkonzentration liegt bei ca. 165.000 PK pro ml Sediment. Der Wiederanstieg von Pinus und des NBP kennzeichnen den Beginn der folgenden PAZ.

\section{W 7 (87-50 cm): Pinus-Secale-Rumex-PAZ}

Die jüngste PAZ zeichnet sich durch hohe Pinus-, Quercus- und NBP-Werte aus. Pinus steigt von zuvor $9 \%$ auf ca. $47 \%$ an, Quercus erreicht fast $35 \%$. Die Anteile von Betula und Alnus fallen am Ende auf Werte unter $10 \%$ zurück. Während die Carpinus-Werte unverändert um $4 \%$ pendeln, gehen die Fagus-Werte bis auf $5 \%$ zurïck.

Die deutliche Zunahme des NBP wird vor allem durch den Anstieg der Poaceae (7,2 \%) hervorgerufen, gefolgt von dem Anstieg der Kulturpflanzen und sekundären Siedlungszeiger. Unter den Kulturpflanzen kommt Secale mit Werten bis zu 3,2 \%, der Getreide indet.Typ mit Werten bis 1,9\% und der Hordeum-Typ mit Werten bis $1 \%$ vor. Vereinzelt tritt der Triticum-Typ auf, Fagopyrum ist mit einem einzigen Pollenkorn belegt.

Die sekundären Siedlungszeiger sind durch erhöhte Anteile von Rumex (2,8\%) und Plantago lanceolata (1,9\%) sowie durch Chenopodiaceae (bis 0,7\%), Centaurea cyanus $(0,5 \%)$, Brassicaceae $(0,2 \%)$ und durch den Humulus/Cannabis-Typ $(0,5 \%)$ vertreten.

Die Kurven der Grünalgen Botryococcus und Pediastrum boryanum zeigen zu Beginn der PAZ Maximalwerte von ca. $20 \%$ bzw. $60 \%$, fallen dann aber im Laufe des Abschnitts zurück auf $10 \%$ bzw. $15 \%$. 
Die durchschnittliche Pollenkonzentration ist erheblich gesunken und beträgt etwa 85.000 PK pro ml Sediment.

\subsection{Krebssee}

Das Pollendiagramm vom Krebssee lässt sich in 7 lokale Pollenzonen (L PAZ) untergliedern. Es beginnt mit dem Ende des Subboreals und endet in der Neuzeit. In der Tabelle 7 sind die lokalen PAZ zur Übersicht zusammengestellt. Im folgenden werden die wesentlichen Veränderungen im Pollendiagramm erläutert.

\section{K 1 (220-173 cm): Betula-Alnus-Plantago lanceolata-PAZ}

Das Gehölzpollenspektrum wird neben Betula mit Werten zwischen 19,3\% und 34,4 \% auch von Alnus $(31,8 \%$ bis 40,3\%) beherrscht. Pinus ist mit ca. $15 \%$ vertreten, Corylus und Quercus erreichen ca. $10 \%$. Ulmus $(1,9 \%$ bis $3,3 \%)$, Tilia $(0,2 \%$ bis $0,8 \%)$ und Fraxinus $(0,4 \%$ bis $1,2 \%)$ sind mit geringen Anteilen präsent. Fagus pendelt zwischen 2,9 \% und 6,1\%, Carpinus ist mit Werten zwischen $2,9 \%$ und 6,8 \% vorhanden.

Das NBP-Spektrum wird dominiert von Poaceae mit zunehmenden Werten zwischen 5,7\% und 15,1\%. Codominant sind Artemisia (5,7\%) und sekundäre Siedlungszeiger, darunter Plantago lanceolata (bis 3,1\%), Rumex (bis 1,7\%) und Chenopodiaceae (bis 5,0\%). Vereinzelt tritt Getreide-Pollen (Getreide indet., Triticum-Typ und Avena-Typ) auf.

Ab $191 \mathrm{~cm}$ liegen erhöhte Werte vom Myriophyllum verticillatum/spicatum-Typ vor. Gleichzeitig steigen die Anteile von Botryococcus (bis 17,5 \%) und Pediastrum boryanum (bis 45,2\%). Tetraedron ist in geringen Mengen ebenfalls vertreten.

Unter den Farnsporen sind Filicinae und Pteridium regelmäßig präsent.

Die Pollenkonzentration steigt allmählich von 4.700 PK pro ml Sediment auf annähernd 120.000 PK pro ml Sediment an.

Die Abgrenzung zur nächsten PAZ erfolgte anhand des Rückgangs des NBP und des Anstiegs der Corylus- und Quercus-Kurve.

\section{K 2a (173-125 cm): Fagus-Carpinus-Cerealia-PAZ}

Während die Anteile von Betula nun tendenziell rückläufig sind (28,9\% bis 15,9\%), herrscht Alnus noch immer mit schwankenden Werten zwischen 17,6\% und 41,5\% vor. Ebenfalls eine abnehmende Tendenz zeigt Pinus. Deutlich angestiegen sind dagegen die 
Anteile von Corylus (bis $19 \%$ ) und Quercus (bis 37,8\%). Ein leichter Rückgang ist bei Ulmus und Tilia zu verzeichnen. Carpinus erreicht nach kontinuierlichem Anstieg bei $145 \mathrm{~cm}$ ein Maximum von 9,7\%. Danach fällt die Kurve auf $7 \%$ zurück. Fagus steigt ab $153 \mathrm{~cm}$ abrupt von 2,8 \% auf 8,5\% an und hält dieses Niveau bis zum Ende der PAZ.

Der Anteil des NBP steigt nach einem Rückgang ab $145 \mathrm{~cm}$ erneut an (bis $22 \%$ ). Hervorgerufen wird dieser erneute Anstieg vor allem durch die Zunahme von Poaceae und Plantago lanceolata auf $16,6 \%$ bzw. $2,2 \%$.

Getreide-Pollen wird häufiger, darunter vereinzelt Secale, der Getreide indet.-Typ und der Triticum-Typ. Letzterer erreicht am Ende $1 \%$. Die Kurven der übrigen terrestrischen Taxa zeigen in dieser PAZ keine signifikanten Veränderungen.

Unter den Sumpf- und Wasserpflanzen kommt es zu einem Rückgang vom Myriophyllum verticillatum/spicatum-Typ.

Das Vorkommen von Farnsporen ist unverändert und bleibt mit Ausnahme der letzten PAZ auf Einzelfunde beschränkt.

Die Grünalgen Pediastrum boryanum und Botryococcus steigen nach einem Rückgang erneut deutlich an und erreichen Werte von $43,6 \%$ bzw. 5,4 \%.

Die durchschnittliche Pollenkonzentration ist auf ca. 300.000 PK pro ml Sediment angestiegen.

Die Abgrenzung zur nächsten PAZ erfolgte anhand des Anstiegs der Carpinus-Kurve.

\section{K 2b (125-101 cm): Betula-Carpinus-PAZ}

Das Baumpollenspektrum wird wieder von Betula und Alnus zu etwa gleichen Teilen (um $30 \%$ ) beherrscht. Pinus zeigt eine leicht zunehmende Tendenz. Die Corylus-Kurve fällt unter $10 \%$, die Quercus-Kurve fällt von 37,8\% auf $12 \%$ zurück. Ulmus und Tilia zeigen sich unverändert. Die Werte von Fagus zeigen einen leichten Rückgang $(8,7 \%$ auf 6,5\%), Carpinus sinkt nach einem Maximum bei $121 \mathrm{~cm}$ von 15,4\% auf 11,9\% zurück.

Die Anteile des NBP fallen von zuvor $22 \%$ auf $10 \%$ zurück. Bedingt wird dies vor allem durch den Rückgang von Poaceae (7 \%) und von Plantago lanceolata (0,5\%). Die Kurve von Rumex zeigt in dieser PAZ eine Unterbrechung. Getreide-Pollen ist nach wie vor nur sporadisch vertreten. 
Unter den Sumpf- und Wasserpflanzen kommt es zu einem Anstieg von Typha, Sparganium, vom Myriophyllum verticillatum/spicatum-Typ und von Nuphar.

Deutlich zurückgegangen sind mit Werten zwischen $0,5 \%$ bis $2 \%$ die Anteile von Pediastrum boryanum und Botryococcus.

Die durchschnittliche Pollenkonzentration ist kaum verändert und beträgt ca. 320.000 PK pro ml Sediment.

Anhand des Rückgangs von Carpinus und anhand des Anstiegs von Fagus und des NBP erfolgt die Abgrenzung zur folgenden PAZ.

\section{K 2c (101-69 cm): Quercus-Fagus-NBP-PAZ}

Das Gehölzpollenspektrum ist durch das Erreichen maximaler Fagus-Werte (13\%) bei $89 \mathrm{~cm}$ gekennzeichnet. Gleichzeitig erreicht auch Quercus ein Maximum (28\%). Betula verhält sich gegenläufig zur Quercus-Kurve und steigt nach einem Rückgang auf 12,6\% erneut auf 26,4\% an. Pinus nimmt stetig zu (bis $15 \%$ ), Corylus fällt nach einem leichten Anstieg wieder auf das Ausgangsniveau (ca. $4 \%$ ) zurück. Die Anteile von Alnus zeigen eine abnehmende Tendenz. Das Spektrum der übrigen EMW-Arten zeigt keine wesentlichen Veränderungen. Fagus fällt nach Erreichen des Maximums auf das Ausgangsniveau $(6,5 \%)$ zurück. Die Carpinus-Werte nehmen kontinuierlich bis 4,4\% ab.

Das NBP-Spektrum verzeichnet einen deutlichen Zuwachs (bis $23 \%$ ), der vor allem durch den Anstieg der Poaceae bis 16,7 \% bedingt ist. Artemisia steigt ab $89 \mathrm{~cm}$ Tiefe auf 3,6\% an. Die Kurve von Rumex setzt wieder ein, Plantago lanceolata ist mit Werten zwischen 1,3\% und $2 \%$ vertreten. Der Anteil der Getreide-PK nimmt kontinuierlich zu, insbesondere der Triticum-Typ (0,6\%) und Secale (1,2\%) sind daran beteiligt. Der Avena- und Hordeum-Typ sind wie bisher nur mit Einzelfunden belegt. Eine leichte Zunahme zeigen die Cyperaceae (bis $3 \%$ ).

Unter den Sumpf- und Wasserpflanzen ist ein Rückgang vom Typha latifolia-Typ und vom Myriophyllum verticillatum/spicatum-Typ zu verzeichnen.

Die Werte der Grünalgen fallen nach einem steilen Anstieg zu Beginn der PAZ gleichmäBig auf das Ausgangsniveau zurück.

Die durchschnittliche Pollenkonzentration ist zurückgegangen und beträgt nur noch 210.000 PK pro ml Sediment.

Die Abgrenzung zur folgenden PAZ orientiert sich an dem Rückgang des NBP. 


\section{K 2d (69-53 cm): Betula-Quercus-PAZ}

Das Baumpollenspektrum wird beherrscht von gleichbleibenden Betula-und QuercusAnteilen (30\% bzw. $17 \%$ ). Pinus pendelt um $12 \%$, Alnus schwankt zwischen $22 \%$ und $30 \%$. Corylus zeigt eine leicht zunehmende Tendenz, Ulmus, Tilia und Fraxinus sind unverändert. Die Anteile von Fagus steigen erneut bis auf $10 \%$ an, während diejenigen von Carpinus konstante Werte um $6 \%$ vorweisen.

Der Anteil des NBP nimmt zunächst geringfügig ab und steigt gegen Ende der PAZ wieder an. Die Poaceae fallen von zuvor $15 \%$ auf $12 \%$, Artemisia kommt nur noch mit Werten zwischen 0,4\% und 1\% vor. Der erneute Anstieg des NBP wird bedingt durch den Anstieg der Getreidewerte, vor allem des Hordeum-Typs (1,4\%) und von Secale (2,8 \%). Plantago lanceolata zeigt nur in der Mitte der PAZ erhöhte Werte. Der Anteil der Cyperaceae schwankt zwischen 2,6\% und 3,2\%.

Das Sumpf- und Wasserpflanzenspektrum tritt erneut der Myriophyllum verticillatum/spicatum-Typ mit erhöhten Werten hervor.

Erhöhte Werte von Tetraedron (23\%) erweitern das Grünalgenspektrum. Pediastrum boryanum und Botryococcus liegen ebenfalls mit erhöhten Werten vor.

Die Pollenkonzentration sinkt kontinuierlich auf ca. 100.000 PK pro ml Sediment.

Die Grenze zur nächsten PAZ ist durch den Wiederanstieg des NBP gekennzeichnet.

\section{K 3a (53-29 cm): Pinus-Secale-Rumex-PAZ}

Ein kontinuierlicher Anstieg von Pinus sowie sehr hohe NBP-Anteile kennzeichnen diese PAZ. Pinus steigt auf ca. $30 \%$ an, Betula pendelt ebenfalls um $30 \%$, Corylus nimmt geringfügig zu, Quercus fällt leicht zurück (17\% auf 11,6\%). Tilia kommt nur noch sporadisch vor, alle übrigen EMW-Arten zeigen sich unverändert. Ein deutlicher Rückgang von 10,1\% auf 2,1\% zeigt sich bei Fagus. Carpinus steigt dagegen erneut auf $9,1 \%$ an.

Der Anteil des NBP erreicht bei $33 \mathrm{~cm}$ rasch Maximalwerte von $110 \%$. Hervorgerufen wird dies vor allem durch die Zunahme von Poaceae (47,2 \%), von Kulturpflanzen (19\%) sowie von sekundären Siedlungszeigern $(25,5 \%)$. Unter den Kulturpflanzen dominiert Secale $(7,9 \%)$ vor dem Triticum-Typ (5,1\%) und dem Hordeum-Typ (3,2\%). Nur vereinzelt ist der Avena-Typ vertreten. Bei den sekundären Siedlungszeigern erreicht Rumex schnell Maximalwerte um $13 \%$, Plantago lanceolata erreicht nur 2,8\%. Ergänzt 
wird das Spektrum der sekundären Siedlungszeiger durch das vermehrte Auftreten von Chenopodiaceae (2,8 \%), vom Humulus/Cannabis-Typ (3,2 \%), von Polygonum aviculare $(1,9 \%)$ und von Urtica $(3,2 \%)$.

Bei $33 \mathrm{~cm}$ zeigt die Cyperaceae-Kurve ein Maximum von $6 \%$.

Die Anteile der Grünalgen steigen auf Maximalwerte an: Botryococcus erreicht 17,8 \%, Pediastrum boryanum $83 \%$ und Tetraedron $23 \%$. Ab $25 \mathrm{~cm}$ ist erstmals Coelastrum $(2,5 \%)$ vertreten.

Die durchschnittliche Pollenkonzentration liegt bei durchschnittlich 40.000 PK pro ml Sediment.

Der Rückgang des NBP kennzeichnet den Übergang zur nächsten PAZ.

\section{K 3b (29-0 cm): Pinus-NBP-PAZ}

Das Pollenspektrum wird von weiter zunehmenden Pinus-Anteilen (bis $36 \%$ ) und fortwährend hohen NBP-Anteilen (ca. $55 \%$ ) dominiert. Die Betula-Werte pendeln um $20 \%$. Corylus und Alnus fallen nach einem Anstieg auf < $10 \%$ bzw. $19 \%$ zurück. Die Querus-Kurve verhält sich gegenläufig und steigt nach einem Rückgang erneut auf $15 \%$ an. Die übrigen EMW-Arten zeigen keine signifikanten Veränderungen. Die Fagus-Werte pendeln nach einem Anstieg zwischen 2,4\% und 4,2 \%, Carpinus hält konstante Werte um ca. $3 \%$.

Die Anteile des NBP sind trotz des Rückgangs noch immer sehr hoch. Der Rückgang wird bedingt durch den Rückgang von Poaceae $(21,2 \%)$, Kulturpflanzen $(7,1 \%)$ und sekundären Siedlungszeigern (10,9\%). Die Anteile vom Triticum-Typ liegen nun zwischen 0,3\% und 1,4\%, diejenigen von Secale schwanken zwischen 2,2 \% und 3,5\%. Die Anteile des Hordeum-Typs sind nur geringfügig gesunken. Unter den sekundären Siedlungszeigern fällt der drastische Rückgang von Rumex (13,6\% auf $4 \%$ ) auf. Polygonum aviculare kommt nur noch vereinzelt vor. Zugenommen haben dagegen die Nachweise vom Humulus/Cannabis-Typ (3,8\%), Urtica schwankt zwischen 0,3\% und $3 \%$. Mit leicht angestiegenen Werten bis $0,8 \%$ ist Centaurea cyanus vertreten. Bei $9 \mathrm{~cm}$ zeigt die Kurve der Filicinae ein Maximum von 4,3\%.

Unter den Sumpf- und Wasserpflanzen tritt bei $17 \mathrm{~cm}$ Sparganium mit einem Maximum $(5,2 \%)$ hervor. Leicht erhöhte Werte liegen ebenfalls vom Myriophyllum verticillatum/spicatum-Typ vor. 
Die Anteile der Grünalgen sind noch immer deutlich erhöht.

In der letzten PAZ steigt die Pollenkonzentration wieder stetig an und erreicht am Ende 120.000 pro ml Sediment.

\subsection{Rybojady}

Das Pollendiagramm von Rybojady wurde in 14 lokale Pollenzonen gegliedert. Es umfasst die gesamte postglaziale Vegetationsentwicklung bis in die heutige Zeit. Eine Übersicht der L PAZ von Rybojady ist in Tabelle 8 zusammengestellt. Im Folgenden werden die wesentlichen Vegetationsveränderungen der einzelnen Abschnitte herausgestellt.

\section{R 1 (883-838 cm): Helianthemum-Salix-Hippophae-PAZ}

Den Hauptbestandteil des Baumpollens bilden Pinus (ca. $45 \%$ ) und Betula (ca. $40 \%$ ). Erweitert wird das Baumpollenspektrum durch geringe Anteile von Hippophae (1,1\% bis $2 \%$ ) und Salix $(0,2 \%$ bis 2,4\%) sowie durch Corylus, Quercus, Tilia, Alnus, Fagus und Carpinus, welche mit geringen Werten vortreten sind.

Der Anteil des NBP beträgt bis zu $20 \%$, darunter ist Helianthemum mit Werten bis zu 9,6 \% am häufigsten vorhanden, danach folgen Poaceae (bis 4,8\%) und Artemisia (bis $1,2 \%)$.

Cyperaceae sind durchgängig mit Werten um $4 \%$ vertreten.

Unter den Sumpf- und Wasserpflanzen tritt Thalictrum mit Werten um 1,5\% hervor.

Unter den Algen kommen die kokkalen Grünalgengattungen Botryococcus und Pediastrum boryanum mit Anteilen bis zu $10 \%$ vor.

Die Pollenkonzentration steigt im Laufe der PAZ von 15.000 PK pro ml auf 80.000 PK pro ml Sediment an.

Die Grenze zur nächsten PAZ ist durch den Rückgang von Hippophae, Salix und Helianthemum gekennzeichnet.

\section{R 2 (838-790 cm): Pinus-Betula-Ulmus-PAZ}

In diesem Diagrammabschnitt übernimmt zunächst Betula mit ansteigenden Werten bis zu $92 \%$ die Vorherrschaft, während Pinus nur mit geringen Werten vertreten ist. Hippophae und Salix sind nur noch selten verhanden. Etwa in der Mitte der PAZ, ab $810 \mathrm{~cm}$, steigt die Pinus-Kurve bis zu $70 \%$ und an, und Betula fällt auf geringe Werte zurück. Gleichzeitig steigen Corylus (bis 12,9\%) und Ulmus (bis 2,4\%) an. Quercus ist in geringen 
Mengen regelmäßig, Tilia nur sporadisch präsent.

Der Anteil der krautigen Taxa ist bis $810 \mathrm{~cm}$ erhöht, bedingt durch den Anstieg von Poaceae (bis 8,4\%), Artemisia (3,0\%), Chenopodiaceae (0,4\%) und Filipendula $(0,6 \%)$. Danach fallen die Kurven wieder auf geringe Werte zurück.

Der Nachweis von Farnsporen beschränkt sich auf leicht erhöhte Anteile von Filicinae.

Der Anteil der Grünalgen nimmt tendenziell ab.

Die durchschnittliche Pollenkonzentration ist erheblich gestiegen und liegt nun bei ca. 200.000 PK pro ml Sediment.

Die Abgrenzung zur nächsten PAZ erfolgt anhand des steilen Anstiegs der Corylus-Kurve von $12,9 \%$ auf $44,7 \%$.

\section{R 3 (790-750 cm): Pinus-Corylus-Quercus-PAZ}

In diesem Abschnitt sind deutliche Vegetationsveränderungen abgebildet: Die CorylusKurve steigt steil bis ca. $100 \%$ an, anschließend fällt sie auf Werte um $50 \%$ zurück.

Pinus steigt nach einem Rückgang wieder auf ca. $70 \%$ an, die Betula-Kurve verhält sich gegenläufig dazu. Ulmus nimmt zu Beginn rasch zu (10,5\%), danach pendeln die Werte um $4 \%$. Quercus steigt kontinuierlich an und erreicht am Ende der PAZ bereits $12 \%$. Tilia ist mit geringen Werten um 0,7 \% regelmäßig präsent.

Der Anteil des NBP fällt nach Erreichen eines Maximums von $10 \%$, das vor allem auf den Anstieg von Calluna zurückzuführen ist, auf Werte zwischen 2,4\% bis 5,5\% zurück.

Es liegen leicht erhöhte Werte von Filicinae vor.

Unter den Grünalgen kommt nur Pediastrum mit leicht erhöhten Werten vor.

Die durchschnittliche Pollenkonzentration liegt in diesem Diagrammabschnitt bei etwa 145.000 PK pro ml Sediment.

Die Abgrenzung zur nächsten PAZ erfolgt anhand des Steilanstiegs der Alnus-Kurve von $4,2 \%$ auf $31,6 \%$.

\section{R 4 (750-694 cm): Alnus-Tilia-PAZ}

Das Baumpollenspektrum wird nun von Alnus mit Werten um $50 \%$ dominiert. Die PinusKurve geht zurück auf Werte $<10 \%$, diejenige von Corylus fällt ebenfalls von zuvor $80 \%$ auf $38 \%$ zurück. Betula pendelt zwischen $20 \%$ und $32 \%$. Quercus ist mit Werten 
zwischen 7,2\% und 9,9\% vertreten, Ulmus steigt auf 9,5\% an. Die Tilia-Kurve zeigt einen steilen Anstieg von 0,7 auf 3,6\%. Fraxinus kommt weiterhin unregelmäßig vor. Regelmäßig dagegen sind nun Hedera und Viscum vertreten.

Der Anteil des NBP pendelt zwischen 2,3-7,4\%. Unter den NBP sowie unter den Farnsporen sind keine signifikanten Veränderungen feststellbar.

Die durchschnittliche Pollenkonzentration liegt mit 190.000 PK pro ml Sediment etwas höher als in der vorangegangenen PAZ.

Der Anstieg der Fraxinus-Kurve markiert den Übergang zur folgenden PAZ.

\section{R 5 (694-534 cm): EMW-Fraxinus-PAZ}

Unter starken Schwankungen $(2,5 \%$ bis $63,5 \%)$ steigt die Pinus-Kurve wieder an, während Betula insgesamt eine abnehmende Tendenz aufweist. Corylus fällt von $90 \%$ auf $35 \%$ zurück. Seit Beginn der PAZ weist Fraxinus eine geschlossene Kurve auf. Quercus steigt rasch auf $15 \%$ an, Ulmus erreicht bei $634 \mathrm{~cm}$ ein Maximum von 12,8\%. Tilia liegt mit Werten zwischen 1,3\% und 4,3\% vor. Während Hedera weiterhin regelmäßig vorkommt, wird Viscum nur noch selten nachgewiesen. Eine deutlich abnehmende Tendenz zeigt die Alnus-Kurve. Etwas häufiger als vorher treten ab nun Pollenkörner von Picea auf.

Der NBP wird ab $602 \mathrm{~cm}$ Tiefe deutlich seltener. Dies ist vor allem durch den Rückgang von Poaceae und von Artemisia bedingt. Letztere ist ab $602 \mathrm{~cm}$ nur noch sehr unregelmäBig nachweisbar.

Unter den Farnsporen tritt vermehrt Pteridium auf.

Die Anteile der Grünalgen Botryococcus und Pediastrum boryanum zeigen keine signifikanten Veränderungen.

Die Pollenkonzentration sinkt von ca. 190.000 PK pro ml auf etwa 60.000 PK pro ml Sediment.

Die Abgrenzung zur nächsten PAZ erfolgt anhand des Rückgangs von Ulmus und des Einsetzens der geschlossenen Fagus-Kurve.

\section{R 6a (534-444 cm): EMW-Fagus-PAZ}

Der Beginn dieser PAZ ist durch den Rückgang von Ulmus von 9,5\% auf 5,3\% gekennzeichnet. Gleichzeitig setzt die geschlossene Kurve von Fagus ein. Pinus (3,9\% 
bis $64,3 \%)$, Betula $(4,6 \%$ bis $27,7 \%)$ und Alnus $(8,3 \%$ bis $51,4 \%)$ unterliegen starken Schwankungen. Die Anteile von Corylus liegen mit Ausnahme von zwei Maxima um $20 \%$. Quercus steigt nach einem Rückgang wieder auf ca. $17 \%$ an. Die Tilia-Kurve pendelt zwischen 1,4\% und 3,8\%. Fagus und Carpinus erreichen ihre empirischen Pollengrenzen bei $514 \mathrm{~cm}$ bzw. $454 \mathrm{~cm}$. Die Anteile liegen bei Fagus um 0,5\% und bei Carpinus um $0,3 \%$.

Das NBP-Spektrum zeigt keine wesentlichen Veränderungen.

Bis auf ein Maximum von Botryococcus $(13,5 \%)$ bei $474 \mathrm{~cm}$ ist das Verhalten der Grünalgen unauffällig.

Die durchschnittliche Pollenkonzentration ist mit 75.000 PK pro ml Sediment wieder leicht angestiegen.

Der Übergang zur folgenden PAZ orientiert sich an dem Anstieg der Fagus- und Carpinus-Kurven.

\section{R 6b (444-350 cm): EMW-Carpinus-Cerealia-PAZ}

Die Kurven von Pinus, Betula und Alnus unterliegen weiterhin starken Schwankungen. Die Corylus-Werte gehen im Laufe der PAZ von ca. $30 \%$ auf $16 \%$ zurück. Quercus steigt nach einem Rückgang wieder auf Werte um $19 \%$. Die Anteile von Ulmus pendeln zwischen $1,5 \%$ und 3,1\%, Tilia bewegt sich auf etwas geringerem Niveau als vorher (1\% und $2 \%$ ), und Fraxinus erreicht nur noch maximal 0,8\%. Acer, Hedera und Viscum werden immer seltener. Während die Anteile von Fagus nur allmählich zunehmen $(1,1 \%$ und 2,5\%), steigen diejenigen von Carpinus bis zu einem Maximum von 9,8\% (bei $362 \mathrm{~cm})$ an. Danach fällt die Carpinus-Kurve auf 4,7 \% zurück.

Das NBP-Spektrum wird erstmals durch vereinzelt auftretende Getreidepollen ergänzt, darunter der Getreide indet.-Typ, der Hordeum-Typ und Secale. Etwas häufiger kommen Rumex (bis 0,4\%), Plantago lanceolata (bis 0,6\%) und Chenopodiaceae (bis 0,3\%) vor.

Farnsporen sind nun so selten, dass die Kurve erstmals Lücken aufweist.

Die telmatischen und aquatischen Taxa sowie die Algen zeigen keine signifikanten Veränderungen.

Die durchschnittliche Pollenkonzentration ist mit ca. 75.000 PK pro ml Sediment unverändert. 
Der Anstieg des NBP und der Rückgang von Quercus kennzeichnen die Grenze zur nächsten PAZ.

\section{R 6c (350-310 cm): Carpinus-Plantago lanceolata-PAZ}

Das Gehölzpollenspektrum weist erhebliche Veränderungen auf. Neben Betula und Corylus sind auch die Werte aller EMW-Arten deutlich rückläufig. Nach den Schwankungen hält Betula ein Niveau von ca. 11,5\%. Corylus fällt erstmals auf Werte $<10 \%$, Quercus zeigt nur einen leichten Rückgang von 13,2\% auf 7,9\%. Ulmus erreicht nur noch maximal 2,6\%, und die Tilia-Kurve fällt unter $1 \%$. Fraxinus ist mit Anteilen zwischen $0,2 \%$ bis $0,5 \%$ nur noch unregelmäßig vertreten. Während die Fagus-Werte zwischen $2 \%$ bis $3 \%$ pendeln, steigen diejenigen von Carpinus rasch zu einem Maximum von $14,1 \%$ an. Danach fällt die Kurve auf 9,4\% zurück.

Unter den krautigen Taxa kommt es zu einem Anstieg von 6,9\% auf 9,5\%, vor allem bedingt durch die Zunahme von Poaceae (2,3\%), Artemisia (2,7\%), Rumex (1,4\%), Plantago lanceolata $(1,1 \%)$ und Chenopodiaceae $(1,1 \%)$.

Unter den Grünalgen weist Pediastrum boryanum deutlich höhere Werte auf (6,6 \%).

Die Pollenkonzentration zeigt eine leicht abnehmende Tendenz. Im Mittel liegt sie bei etwa 57.000 PK pro ml Sediment.

Der Übergang zur nächsten PAZ wird durch den Rückgang des NBP charakterisiert.

\section{R 7a (310-278 cm): Betula-Carpinus-PAZ}

Die Werte von Betula steigen von 8,4\% auf 28,8 \% und dominieren zusammen mit noch immer hohen Alnus-Anteilen (19,3\% bis 50,1\%) das Baumpollenspektrum. Die PinusKurve verhält sich gegenläufig zur Betula-Kurve und fällt von 26,6 \% auf 2,7 \%. Corylus steigt vorübergehend auf $11,9 \%$ an, fällt danach wieder auf $6 \%$ zurück. Quercus zeigt sich kaum verändert. Die Anteile von Ulmus, Tilia und Fraxinus weisen dagegen erneut einen Rückgang auf. Fagus steigt von $3 \%$ auf 4,7\% an, Carpinus ist mit deutlich höheren Anteilen zwischen $7,7 \%$ und $10 \%$ vertreten.

Bedingt durch den Rückgang der Poaceae, Artemisia, Rumex, Plantago lanceolata und Chenopodiaceae zeigt die NBP-Kurve einen leichten Rückgang. Der Nachweis von Getreide beschränkt sich auf einen Einzelfund vom Hordeum-Typ.

Das Spektrum der Sumpf- und Wasserpflanzen ändert sich, abgesehen von einem vermehrten Vorkommen von Nymphaea $(1,7 \%)$ nicht wesentlich. 
Unter den Algen kommt es zu einem Anstieg von Botryococcus, während Pediastrum boryanum das Niveau der vorangegangenen PAZ beibehält.

Die durchschnittliche Pollenkonzentration ist wieder etwas angestiegen und beträgt nun ca. 67.000 PK pro ml Sediment.

Die Grenze zur nächsten PAZ wurde anhand des erneuten Anstiegs der NBP-Kurve festgelegt.

\section{R 7b (278-238 cm): Quercus-Carpinus-NBP-PAZ}

Das Gehölzpollenspektrum zeigt bis auf den Anstieg von Pinus auf $27 \%$ und den erneuten Rückgang von Betula und Alnus nur geringfügige Veränderungen. Die Anteile von Quercus pendeln nach einem leichten Anstieg um etwa $14 \%$, Ulmus zeigt sich unverändert, und die Tilia-Kurve setzt zum ersten Mal aus. Fagus und Carpinus halten das Niveau der vorherigen PAZ.

Ein deutlicher Anstieg ist bei den krautigen Arten zu verzeichnen. Poaceae (bis 2,2 \%), Artemisia (bis 6,0\%), Kulturpflanzen und sekundäre Siedlungszeiger bedingen diesen Anstieg. Unter den Kulturpflanzen tritt neben erhöhten Anteilen vom Triticum-Typ (bis $0,5 \%$ ) und Getreide indet.-Typ Secale mit regelmäßiger Präsenz (bis 0,3\%) hervor. Rumex (1\%), Plantago lanceolata (1,1\%), Chenopodiaceae (0,8\%), Brassicaceae $(1,4 \%)$, Humulus/Cannabis $(0,6 \%)$ und Polygonum aviculare $(0,3 \%)$ sind ebenfalls häufiger vorhanden als vorher. Außerdem erweitert sich das Spektrum des übrigen NBP um vermehrte Nachweise von Caryophyllaceae sowie verschiedener Asteraceae.

Neben den Cyperaceae erweitert sich das Spektrum der Hygrophyten um vereinzelt vorkommende Pollen von Menyanthes, vom Potentilla-Typ, vom Lythrum-Typ, vom Mentha-Typ und von Valeriana.

$\mathrm{Ab} 250 \mathrm{~cm}$ zeigt sich eine Zunahme von Pediastrum boryanum (bis 36,8 \%).

Mit durchschnittlich 80.500 PK pro ml Sediment setzt sich die ansteigende Tendenz der Pollenkonzentration fort.

Der Rückgang der NBP-Kurve grenzt diese PAZ von der folgenden ab.

\section{R 7c (238-214 cm): Quercus-Carpinus-PAZ}

Diese PAZ ist durch einen leichten Anstieg von Quercus (17,3\%) und einen Anstieg von Carpinus auf 13,3\% gekennzeichnet. Betula und Alnus weisen zunächst eine abnehmende 
Tendenz auf, steigen aber gegen Ende der PAZ erneut an. Die Pinus-Werte schwanken erneut zwischen 6,2\% und 52,3\%. Ulmus, Tilia, Fraxinus und Fagus zeigen keine signifikanten Veränderungen.

Die Kurve des NBP fällt auf geringe Werte um $5 \%$ zurück. Dieses wird hervorgerufen durch den Rückgang von Poaceae und Artemisia sowie durch den Rückgang der Kulturpflanzen und der sekundären Siedlungszeiger. Ebenso setzt der Nachweis übriger krautiger Taxa fast vollständig aus.

Die Werte der Grünalge Pediastrum boryanum steigen nach einem Rückgang auf 6,2\% erneut auf $28 \%$ an.

Bemerkenswert ist die deutliche Zunahme der Pollenkonzentration mit Werten um 128.000 PK pro ml Sediment.

Der Übergang zur folgenden PAZ ist durch den Wiederanstieg des NBP gekennzeichnet.

\section{R 7d (214-105 cm): Pinus-Quercus-NBP-PAZ}

Dieser Abschnitt ist gekennzeichnet durch einen sukzessiven Rückgang von Quercus, Fagus und Carpinus. Der Anteil von Pinus steigt unter Schwankungen an. Betula fällt nach einem Anstieg auf Werte um $10 \%$ zurück. Ulmus kommt mit Werten zwischen $0,1 \%$ und $1,3 \%$ regelmäßig vor, Tilia ist nur noch sehr sporadisch vertreten. Fagus fällt auf Werte zwischen 1,3\% und 2,3\% zurück, die Anteile von Carpinus steigen nach einem Rückgang auf $3 \%$ ab $158 \mathrm{~cm}$ wieder auf $7 \%$ an. Die Alnus-Kurve zeigt einen parallelen Verlauf bei insgesamt höheren Werten. Hier fallen die Werte von $40 \%$ auf $16,1 \%$ und steigen dann wieder auf $34 \%$ an.

Die Anteile des NBP nehmen bis $178 \mathrm{~cm}$ von $8,4 \%$ auf $23,6 \%$ zu und fallen danach auf $11,8 \%$ zurück. Getreide indet. und Secale sowie Rumex, Plantago lanceolata und Chenopodiaceae treten zu Beginn und gegen Ende des Abschnitts mit erhöhten Werten hervor. Erstmals konnte Pollen vom Avena-Typ nachgewiesen werden. Unter den Ericaceae kommt es ab $158 \mathrm{~cm}$ zu einer Zunahme von Vaccinium. Die Cyperaceae, deren Werte überwiegend zwischen $1 \%$ und $2 \%$ pendeln, zeigen ab $186 \mathrm{~cm}$ einen kurzfristigen Anstieg auf 11,8\%, gleichzeitig setzt die Kurve von Menyanthes ein.

Unter den Sumpf- und Wasserpflanzen kommt es ab $186 \mathrm{~cm}$ zu einem vermehrten Auftreten vom Typha latifolia-Typ (bis 5,7 \%) und von Utricularia. Ebenfalls ab $186 \mathrm{~cm}$ setzt der Nachweis der Farnsporen und Grünalgen nahezu aus. 
Die Pollenkonzentration sinkt bis auf 20.000 PK pro ml Sediment.

Der Übergang zur folgenden PAZ orientiert sich an dem erneuten Anstieg des NBP.

\section{R 8a (105-75 cm): Pinus-Quercus-PAZ}

Schwankende, aber dennoch parallel verlaufende Baumpollenkurven charakterisieren diesen Abschnitt, so dass sich weder eine ab- noch eine zunehmende Tendenz erkennen lässt. Die Pinus-Kurve verhält sich gegenläufig zu den übrigen Baumpollenkurven.

Ein deutlicher Anstieg (bis $22 \%$ ) zeigt sich bei NBP, hervorgerufen durch die Zunahme der Kulturpflanzen, darunter der Getreide indet.-Typ (1,7 \%), der Hordeum-Typ (0,8 \%), der Triticum-Typ (0,3\%) und Secale (1,2\%). Gleichzeitig nimmt auch der Anteil sekundärer Siedlungszeiger stetig zu. Erstmals kommen Centaurea cyanus mit Werten um $0,5 \%$ und Convolvulus arvensis $(0,1 \%)$ vor. Häufiger als zuvor sind mit Anteilen um 0,9\% Caryophyllaceae und Asteraceae Liguliflorae vertreten. Unter den Ericaceae macht sich ein leichter Anstieg von Calluna (bis 4,4\%) und Vaccinium (bis 2,3\%) bemerkbar. Die Anteile der Cyperaceae (5,4\%) und von Menyanthes $(0,5 \%)$ sind ebenfalls wieder leicht angestiegen.

Grünalgen sind nur noch in geringen Mengen vertreten.

Die Pollenproduktion ist wieder auf 75.000 PK pro ml Sediment angestiegen.

Der Steilanstieg des NBP kennzeichnet den Übergang zur jüngsten PAZ.

\section{R 8b (75-0 cm): Pinus-NBP-PAZ}

Die Schwankungen des vorangegangenen Abschnitts lassen nach, und Pinus dominiert nun mit Werten um $40 \%$ das Baumpollenspektrum. Betula und Alnus steigen nach einem Rückgang ab $16 \mathrm{~cm}$ wieder an. Bei allen übrigen Baumpollentaxa zeigt sich ein stark rückläufiger Trend. Die Anteile von Quercus sinken auf 4,1\%, diejenigen von Ulmus, Fagus und Carpinus liegen zwischen 0,1\% und 0,4\%. Mit geringen Werten ist Salix wieder regelmäßig vertreten.

Der Anteil des NBP erreicht bei $16 \mathrm{~cm}$ ein Maximum von fast $80 \%$, danach fällt die Kurve auf $32 \%$ zurück. Hervorgerufen wird der Anstieg des NBP vor allem durch die Zunahme von Cyperaceae auf 62,2\% sowie durch den Anstieg der Kulturpflanzen und sekundären Siedlungszeiger. Mit Anteilen von 5,9\% ist Secale am häufigsten, gefolgt von Getreide indet. und vom Triticum-Typ (beide 1,6\%), vom Hordeum-Typ (1,4\%), AvenaTyp $(0,3 \%)$ und Fagopyrum $(0,2 \%)$. Unter den sekundären Siedlungszeigern fallen 
besonders Rumex (2,1\%), Plantago lanceolata (2,6\%), Chenopodiaceae (2,1\%), Brassicaceae $(0,8 \%)$ und Centaurea cyanus $(0,8 \%)$ durch erhöhte Werte auf. Erstmals kommt auch Pollen vom Trifolium-Typ vermehrt vor. Unter den übrigen krautigen Taxa liegen erhöhte Anteile von Caryophyllaceae, Ranunculaceae und verschiedenen Asteraceae vor.

Das Sumpf- und Wasserpflanzenspektrum wird durch einen erhöhten Anteil von Thalictrum 5,2 \%) und Sparganium $(0,2 \%)$ repräsentiert.

$\mathrm{Ab} 24 \mathrm{~cm}$ kommt es zu einem steilen Anstieg von Sphagnum-Sporen bis zu $90 \%$. Dieser Anstieg korreliert mit einem Rückgang der Grünalgen.

Die Pollenproduktion in der jüngsten PAZ steigt nach einem Rückgang bis zu 30.000 PK pro $\mathrm{ml}$ wieder auf das vorherige Niveau (75.000 PK pro $\mathrm{ml}$ ) an.

\subsection{Pawski Ług}

Das Pollendiagramm vom Pawski Ług wurde in acht lokale Pollenzonen (L PAZ) gegliedert, die in Tabelle 9 zur Übersicht zusammengestellt sind. Das Pollendiagramm beginnt mit dem Älteren Atlantikum und reicht bis ins Jüngere Subatlantikum. In fast allen Abschnitten liegt eine starke Repräsentanz von Pinus-Pollen vor. In der Regel wird der Pollen der überrepräsentierten Taxa, der meistens aus dem direktem Umfeld der Ablagerungsstätte stammt, in der dem Pollenprozentdiagramm zugrunde liegenden Grundsumme nicht berücksichtigt. Dadurch wird verhindert, dass der überrepräsentierte Pollenanteil, der indirekt die Anteile übriger Taxa verringert (Summe BP=100\%), bei der Auswertung des Pollendiagramms zu Fehlinterpretationen hinsichtlich der regionalen Vegetationsentwicklung führt. Das Pollendiagramm vom Pawski Ług basiert jedoch trotz des durchgängig hohen Pinus-Anteils auf einer Grundsumme inklusive Pinus. Auf diese Weise wird der Vergleich mit anderen Pollendiagrammen vereinfacht. Bei der Interpretation der Befunde gilt es jedoch, dies besonders zu berücksichtigen.

\section{P 1 (665-640 cm): Pinus-EMW-PAZ}

Hohe Pinus-Werte zwischen $65 \%$ und $80 \%$ dominieren das Gehölzpollenspektrum dieser PAZ.

Kodominant, wenn auch mit deutlich geringeren Werten um $14 \%$, ist Quercus. Die Anteile von Betula und Corylus liegen um $7 \%$. Tilia und Ulmus sind mit Werten um $2 \%$ 
bzw. $3 \%$ präsent. Die Alnus-Kurve steigt auf $6 \%$ an. Viscum und Hedera sind mit geringen Werten vorhanden.

Der Anteil des NBP liegt bei 6,8\%, darunter treten vor allem die Poaceae $(2,8 \%)$ und Cyperaceae $(2,4 \%)$ mit erhöhten Werten hervor.

Unter den Sumpfpflanzen tritt Sparganium vermehrt auf.

Grünalgen sind im gesamten Pollendiagramm nur sporadisch vertreten.

Die durchschnittliche Pollenkonzentration liegt bei ca. 120.000 PK pro ml Sediment.

Die Abgrenzung zur nächsten PAZ erfolgte anhand des Anstiegs der Fraxinus-Kurve.

\section{P 2 (640-592 cm): Pinus-EMW-Fraxinus-PAZ}

Weiterhin beherrschen hohe Pinus-Anteile (um 65\%) das Baumpollenspektrum. Die Betula- und Corylus-Werte pendeln um $10 \%$. Während Quercus auf $6 \%$ zurück fällt, steigt Ulmus bis zu 4,5\% an. Tilia verhält sich unverändert. Fraxinus ergänzt das EMWSpektrum und steigt schnell auf $1,9 \%$ an, fällt danach jedoch auf Werte um 0,6 \% zurück.

Die Anteile des NBP liegen nun bei ca. 2,5\%.

Unter den Sumpfpflanzen wird Sparganium seltener, dafür kommt Thalictrum etwas häufiger vor.

Der Anteil der Pteridium-Sporen nimmt im Laufe des Abschnitts ab. Ein deutlicher Anstieg bis zu $10 \%$ ist bei den Sphagnum-Sporen zu verzeichnen.

Die Anteile der Mikrofossilien Amphitrema und Assulina steigen bei $600 \mathrm{~cm}$ auf 31,7\% bzw. $5,1 \%$ an.

Die Pollenkonzentration nimmt kontinuierlich zu und erreicht am Ende ca. 200.000 PK pro $\mathrm{ml}$ Sediment.

Der Übergang zur nächsten PAZ wird durch den Rückgang von Ulmus von 4,7\% auf $1,9 \%$ charakterisiert.

\section{P 3a (592-496 cm): Pinus-Fagus-Carpinus-PAZ}

Pinus ist unverändert stark präsent. Betula pendelt mit Ausnahme zweier Maxima (30\% und $23 \%$ ) um $10 \%$. Die Corylus- und die Quercus-Kurve steigen unter Schwankungen tendenziell an, diejenige von Ulmus fällt nach einem Anstieg (4,5\%) auf 0,5\% zurück. Die Anteile von Tilia pendeln um 1,5\%. Fraxinus nimmt kontinuierlich bis auf 0,1\% ab. 
Alnus weist gegen Ende der PAZ ein kurzfristiges Maximum von $41 \%$ auf. Fagus erreicht bei $552 \mathrm{~cm}$ mit Werten bis zu 1,2\% die empirische Pollengrenze, leicht verzögert, ab $536 \mathrm{~cm}$, schließt sich die Carpinus-Kurve.

Der Anteil der NBP-Werte unterliegt Schwankungen zwischen 1,9\% bis 7,7\%. Eine deutliche Zunahme zeigen die Anteile von Calluna mit Werten bis 3,7\%. Farnsporen, darunter Pteridium und Filicinae, sind noch immer regelmäßig vertreten.

Der Anteil der Sphagnum-Sporen fällt nach einem Anstieg $(38,4 \%)$ wieder auf geringe Werte zurück. Mikrofossilien treten nur in geringen Mengen auf.

Die Pollenkonzentration fällt von anfangs 480.000 PK pro ml auf knapp 100.000 PK pro ml zurück.

Die Abgrenzung zur nächsten PAZ erfolgt anhand des Auftretens von Plantago lanceolata.

\section{P 3b (496-400 cm): Pinus-Fagus-Carpinus-Plantago lanceolata-PAZ}

Das Gehölzpollenspektrum wird weiterhin von Pinus beherrscht. Die Betula-Werte steigen nach einem leichten Rückgang erneut auf Werte um $10 \%$ an. Corylus und Quercus verhalten sich tendenziell rückläufig. Ulmus ist mit Werten um 1,5\% vertreten, Tilia ist nach einem Rückgang von 1,4 \% nur noch mit Werten $<1 \%$ präsent. Die AlnusKurve zeigt sich unverändert. Die Fagus-Anteile steigen kontinuierlich bis auf 2,6\% an, Carpinus dagegen steigt bei $456 \mathrm{~cm}$ sprunghaft von zuvor $0,4 \%$ auf $2,2 \%$ und erreicht am Ende bereits $4,1 \%$.

Der Anteil des NBP fällt von einem anfänglichen Maximum (12,6\%) auf Werte um 2,5\% zurück, steigt gegen Ende der PAZ erneut auf 8,9\% an. Bedingt wird dies durch den Anstieg von Artemisia. Sekundäre Siedlungszeiger, wie Plantago lanceolata $(0,3 \%)$ und Rumex $(0,1 \%)$ sind seit Beginn der PAZ regelmäßig vertreten. Chenopodiaceae kommen ebenfalls vermehrt vor.

Unter den Sumpfpflanzen tritt Sparganium wieder vermehrt in Erscheinung, während Thalictrum seltener wird. Alle übrigen Taxa verhalten sich unauffällig.

Die Pollenkonzentration schwankt nun zwischen 100.000 und 150.000 PK pro ml.

Die Abgrenzung zur nächsten PAZ erfolgte anhand des Rückgangs von Corylus und des Einsetzens der Getreide-Kurve. 


\section{P 4a (400-256 cm): Pinus-Fagus-Carpinus-Cerealia-PAZ}

Unverändert ist die Dominanz von Pinus innerhalb des Gehölzpollenspektrums. Die Betula-Kurve steigt tendenziell an, diejenige von Corylus pendelt nach dem Rückgang nur noch um $3 \%$. Die Anteile von Quercus nehmen ab $312 \mathrm{~cm}$ erneut leicht zu, die Anteile von Ulmus $(0,5 \%)$, Tilia $(0,3 \%)$ und Fraxinus $(0,1 \%)$ sind nochmals weiter gesunken. Fagus erreicht bei $296 \mathrm{~cm}$ Werte von $4 \%$, Carpinus steigt an dieser Stelle zu einem Maximum von $7 \%$ an und fällt danach auf 2,8\% zurïck.

Der Anteil des NBP fällt kontinuierlich zurück (bis 1,7\%). Ab $376 \mathrm{~cm}$ tritt erstmals vereinzelt Pollen vom Getreide indet.-Typ in Erscheinung. Die zuvor noch regelmäßig vorhandenen Siedlungszeiger treten seltener auf.

Das Sumpfpflanzenspektrum zeigt sich unverändert.

Unter den Sporen ist zwischen $312 \mathrm{~cm}$ und $280 \mathrm{~cm}$ ein deutlicher Anstieg von SphagnumSporen (bis $50 \%$ ) zu verzeichnen. Mikrofossilien sind nur zu Beginn der PAZ (360 cm) in erhöhter Anzahl vorhanden.

Die Pollenkonzentration schwankt zwischen 50.000 bis 200.000 PK pro ml Sediment.

Der Anstieg der Carpinus-Kurve kennzeichnet den Übergang zur nächsten PAZ.

\section{P 4b (256-224cm) Pinus-Carpinus-PAZ}

Das Gehölzpollenspektrum wird zum ersten Mal durch einen Rückgang der Pinus-Anteile von $61,1 \%$ auf $41,7 \%$ gekennzeichnet. Gleichzeitig steigt der Anteil von Betula (bis 10,5\%), Alnus (bis 21,8\%), Carpinus (bis 10,7\%) und Fagus (4,5\%). Quercus, Tilia und Fraxinus zeigen sich unverändert.

Das Spektrum des NBP weist keine signifikanten Veränderungen auf.

Unter den Farnsporen kommt es zu einer leichten Zunahme von Pteridium.

Die Pollenkonzentration liegt durchschnittlich bei 45.000 PK pro ml Sediment.

Die Abgrenzung zur nächsten PAZ erfolgte anhand des Rückgangs der Carpinus-Kurve.

\section{P 4c (224-124 cm): Pinus-Betula-PAZ}

Die Pinus-Kurve steigt nach dem Rückgang erneut auf $65 \%$ an. Betula setzt den ansteigenden Trend fort und erreicht am Ende des Diagrammabschnitts Anteile um 28,3\%. Die EMW-Arten zeigen sich unverändert. Die Alnus-Kurve zeigt bis $168 \mathrm{~cm}$ einen kontinuierlichen Rückgang (bis 2,7\%), danach steigt sie wieder bis auf $32 \%$ an. 
Die Kurven von Fagus und Carpinus zeigen ebenfalls einen rückläufigen Trend. Fagus fällt von 4,5\% auf durchschnittlich 2,5\% zurück, die Carpinus-Werte pendeln nach einem Rückgang um 3,5\%.

Der Anteil des NBP ist zu Beginn der PAZ durch die Zunahme von Poaceae und Artemisia sowie durch den Anstieg vom Hordeum-Typ (bis 0,9\%), von Rumex (bis 0,8 \%) und von Plantago lanceolata (bis 0,5 \%) leicht erhöht. Zum ersten Mal tritt Pollen vom Triticum-Typ und von Secale in Erscheinung.

Die Anteile der übrigen terrestrischen Kräuter zeigen keine signifikanten Veränderungen. Farnsporen treten nur noch sporadisch auf.

Die Pollenkonzentration ist gegenüber dem vorangegangenen Diagrammabschnitt bis auf eine Ausnahme (bei $168 \mathrm{~cm} 125.000 \mathrm{PK}$ pro ml) unverändert.

Die Abgrenzung zur nächsten PAZ ist durch den Anstieg des NBP gekennzeichnet.

\section{P 5 (124-104 cm): Betula-Alnus-NBP-PAZ}

Zum zweiten Mal ist die Dominanz von Pinus durch einen Rückgang auf 9,7 \% vorübergehend aufgehoben. Statt dessen dominieren Betula (bis 47,1 \%) und Alnus (bis 36,5 \%) das Baumpollenspektrum. Die Kurven von Corylus (bis 9,1\%), Quercus (bis 13,7 \%) und Ulmus (bis 1,5\%) steigen ebenfalls erneut an. Nach einem Anstieg auf 3,7\% sinken die Fagus-Werte auf 1,5\%, die Carpinus-Werte fallen nach dem Anstieg auf 6,9\% rasch auf $0,9 \%$ zurück.

Der Anteil des NBP erreicht mit 16,4 \% maximale Werte. Hervorgerufen wird dieser hohe Anteil durch die Zunahme von Kulturpflanzen und sekundären Siedlungszeigern, insbesondere von Plantago lanceolata (1,8\%), Rumex $(1,8 \%)$ und Centaurea cyanus $(0,7 \%)$. Unter den Kulturpflanzen sind der Getreide indet.-Typ, der Hordeum-Typ und Secale mit erhöhten Werten präsent, während der Triticum-Typ nur einmal nachgewiesen wurde. Des weiteren kommt es zu einem vermehrten Vorkommen von Ericaceae $(8,4 \%)$ sowie zu einem Anstieg von Empetrum (2,3\%) und Vaccinium (1,1\%).

In der jüngsten Probe ist der Anteil der Sphagnum-Sporen mit 5,1 \% leicht erhöht.

Die durchschnittliche Pollenkonzentration liegt bei ca. 33.000 PK pro ml Sediment. 


\subsection{Synchronisation der lokalen Pollenzonen (L PAZ) mit waldgeschichtlichen}

Abschnitten

In der Abbildung 5 ist die Synchronisation der lokalen PAZ der verschiedenen Standorte dargestellt. In den Tabellen 6 bis 9 ist die auf calibrierten ${ }^{14} \mathrm{C}$-Altern beruhende zeitliche Stellung der PAZ angegeben.

Wie die Gegenüberstellung der lokalen PAZ der vier Untersuchungsgebiete zeigt, ist eine Synchronisierung nur bedingt möglich. Bis zum Subboreal zeigen die lokalen Pollenzonen vom Wustrowsee (W 1-4), Rybojady (R 2-5) und Pawski Ług (P 1-2) zwar biostratigraphisch sehr gute Übereinstimmungen, so dass man sie durchaus als regionale PAZ bezeichnen kann, chronologisch lassen sie sich jedoch nicht parallelisieren. Ab dem Subboreal ist, wie die lokalen PAZ belegen, die Vegetationsentwicklung an allen vier Standorten so unterschiedlich, dass eine Synchronisation nur noch bedingt möglich ist. Während des Älteren Subatlantikums stimmen zumindest die L PAZ der Lokalitäten aus dem gleichen Untersuchungsgebiet zum großen Teil überein. 


\section{Die Vegetationsentwicklung entlang des östlichen unteren Odertals und südlichen unteren Wartatals}

Im folgenden wird die Entwicklung der Vegetation im Umkreis der vier untersuchten Lokalitäten beschrieben.

\subsection{Die holozäne Vegetationsentwicklung am Wustrowsee und Krebssee}

\subsubsection{Präboreal (IV)}

Die pollenanalytischen Befunde der basalen Sedimente des Wustrowsees spiegeln eindeutig den Beginn der postglazialen Vegetationsentwicklung wider, obwohl die Radiokarbondatierungen aus diesem Abschnitt, der Chronologie von MANGERUD et al. (1974) folgend, eindeutig eine ältere, spätglaziale Altersstellung, nämlich Alleröd, implizieren. Gegen die Zuordnung der ältesten L PAZ (W 1) zur Alleröd-Zeit im Sinne von FIRBAS (1949) spricht das Fehlen eindeutiger Bioindikatoren, wie z. B. die Ausbreitung der Kiefern, die nach BRANDE (1980b) kennzeichnend sind für das Alleröd. Die Jüngere Dryas, die in den meisten Pollendiagrammen Mitteleuropas durch hohe Juniperus- und Artemisia-Werte charakterisiert ist (BRANDE 1980b; TOBOLSKI \& OKUNIEWSKANOVACZYK 1989; LiTT 1994; LiTT \& STEBICH 1999; MiLECKA 2001b), ist ebenfalls aufgrund des geringen Vorkommens beider Taxa im vorliegenden Pollendiagramm nicht eindeutig belegt. Statt dessen kennzeichnen hohe Betula-Werte den ältesten Vegetationsabschnitt W 1, d. h. Baumbirken waren bereits fester Bestandteil der Vegetation. Der Polleninflux der Kiefer (bis maximal $10.000 \mathrm{PK} / \mathrm{cm}^{2 *}$ a) belegt, dass diese sich bereits im Untersuchungsgebiet etabliert hatte, obwohl die Anteile im Prozentdiagramm noch vergleichsweise gering ausfallen (AMMANN 1984). Ein durch Umlagerung bedingter Anteil von Pinus ist vermutlich auszuschließen. Das Fehlen bzw. das nur vereinzelte Vorkommen heliophiler Sträucher wie Hippophae und Juniperus sowie die geringen Werte des NBP deuten darauf hin, dass die Sedimentation in das Seebecken einsetzte, als die Vegetationsentwicklung schon so weit fortgeschritten war, dass die spätglaziale strauch- und krautreiche Tundrenvegetation bereits durch lichten borealen BirkenKiefernwald verdrängt worden war. Der geringe minerogene Anteil in den überwiegend aus Kalkdetritusmudde bestehenden Sedimenten in diesem Abschnitt spricht für eine überwiegend geschlossene Vegetationsdecke. Die tendenziell abnehmenden Werte des heliophilen Beifuß (Artemisia) deuten auf die zunehmende Beschattung des Bodens 
infolge der voranschreitenden Bewaldung. Ein weiterer Beleg für die Zunahme der Bewaldung ist der kontinuierliche Anstieg der Pollenkonzentration.

Das geringe und im Laufe von W 1 nachlassende Vorkommen des Spätglazial-Indikators Empetrum ist einerseits ein weiterer Beleg für die Einordnung ins Postglazial, andererseits unterstreicht es, da Empetrum nach FIRBAS (1949) von Westen nach Osten abnimmt, die zunehmende Kontinentalität in dieser Region. Allerdings ist der Rückgang von Empetrum laut BRANDE (1980b) nicht ausschließlich edaphisch bedingt.

Die Einwanderung von Ulmus und Corylus aus ihren Glazialrefugien, die nach LANG (1994) auf dem Apennin, dem Balkan und vermutlich auch der Iberischen Halbinsel zu suchen sind, dokumentiert die fortschreitende Erwärmung im Präboreal. $\mathrm{Ab}$ ca. 8.200 cal. B.C. waren vereinzelt Ulmen im Untersuchungsgebiet verbreitet. Die Anwesenheit der Ulme in den Wäldern um den Wustrowsee ist ab 7.700 cal. B.C. durch das Erreichen von Prozentanteilen von mehr als $2 \%$ eindeutig belegt (vgl. HUNTLEY \& BIRKS 1983). Zur gleichen Zeit erfolgte die Ausbreitung der Hasel. Anhand der geschlossenen Kurven von Ulmus und Corylus lässt sich das Präboreal u. a. nach BRANDE (1980) in zwei Abschnitte gliedern, die sich auch im Pollendiagramm vom Wustrowsee widerspiegeln. Trotz der Ausbreitung der thermophilen Hölzer blieb am Wustrowsee die Birke, gefolgt von der Kiefer, die dominierende Baumart, wohingegen in den meisten Pollendiagrammen aus den westlichen Nachbarregionen Brandenburgs und Berlins die Kiefer als beherrschende Baumart hervortrat (MÜLLER 1969; Brande 1980b). Eine Ausnahme bildet das Oderbruch, das nach JAHNS (2000) ebenfalls eine präboreale Birkendominanz aufweist. Nach ToBOLSKI (1990), RALSKA-JASIEWICZOWA et al. (1998) und MAKOHONIENKO (2001b) herrschten in den präborealen Wäldern der großpolnisch-kujawischen Ebene ebenfalls Birken vor.

Eine präboreale Abkühlungs-Phase, die in verschiedenen Pollendiagrammen durch den Rückgang wärmeliebender Arten wie z. B. Populus und Filipendula sowie dem gleichzeitigen Anstieg lichtbedürftiger Arten aufgrund einer kältebedingten Auflichtung der Wälder nachweisbar ist (vgl. BeHRE 1978; BITTMANN \& PASDA 1999; MERKT \& MülleR 1999), zeichnet sich im vorliegenden Pollendiagramm einerseits aufgrund des zu großen Probenabstandes $(8 \mathrm{~cm},>160$ Jahre $)$ und andererseits aufgrund des Mangels an geeigneten Indikatoren nicht ab.

Für die Uferbereiche des Wustrowsees werden Verlandungsgesellschaften mit Cyperaceae, Typha latifolia, Sparganium, Myriophyllum und Polypodiaceae angenommen. 


\subsubsection{Boreal (V)}

Während des Boreals, das sich im Pollendiagramm durch den Abschnitt W 2 widerspiegelt und dessen Beginn am Wustrowsee auf 8.700 cal. B.C. datiert, schritt die Ausbreitung der thermophilen, mesokratischen Hölzer weiter fort. Besonders rasch breitete sich die Hasel in den bestehenden Birken-Kiefern-Wäldern aus, wie der steile Anstieg der Haselwerte (> $200 \%$ ) belegt. Die extrem hohen Hasel-Werte am Wustrowsee übertreffen bei weitem diejenigen von Rybojady und von Pawksi Lug sowie der benachbarten Regionen westlich und östlich des unteren Oderlaufs (vgl. BRANDE 1980b; ENDTMANN 1998; RALSKAJASIEWICZOWA et al. 1998; MAKOHONIENKO 2001b; JAHNS et al. 2002; WOLTERS 2002). Es ist anzunehmen, dass im Bereich der Endmoränenzüge die Standortbedingungen für die Hasel günstiger waren als in den Sandergebieten. Allerdings scheinen nicht nur edaphische Gründe für die unterschiedliche Haselpräsenz verantwortlich zu sein, wie bereits WOLTERS (2002) kritisch anmerkte, da auch aus Sandergebieten hohe boreale Hasel-Werte vorliegen (vgl. KIRK 1960). Zu den bevorzugten Haselstandorten zählten sicher auch die Seeufer. Dies würde zum Teil die hohe Präsenz von Haselpollen im Pollendiagramm erklären. Darüber hinaus breitete sich die Hasel im Unterholz der Birken-Kiefern-Wälder aus, wodurch nach SCAMONI (1988) die Verjüngung der Kiefernwälder vermindert wurde.

Auf den mäßig trockenen Standorten mischte sich die Eiche, deren Anwesenheit seit 7.000 cal. B.C. belegt ist, unter die Kiefernbestände. Die Ausbreitung der Eichen erfolgte ca. 6.700 cal. B.C.. Zur gleichen Zeit setzte die Etablierung der Linde, die frische bis mäßig trockene, basenreiche Böden bevorzugt (OBERDORFER 1994), ein. Die Kiefer wurde demnach einerseits indirekt durch die Hasel, anderseits durch die Ausbreitung der thermophilen Laubhölzer auf die Standorte verdrängt, die den Randbereichen ihrer ökologischen Amplitude zuzuordnen sind, nämlich auf die nährstoffarmen nassen und trockenen Böden (vgl. ELLENBERG 1996).

Bereits von Beginn des Boreals an war die Erle im Untersuchungsgebiet anwesend. Eine stärkere Ausbreitung setzte allerdings erst im jüngeren Teil des Boreals ein, und die Erle verdrängte die Birke von den grundwassernahen Standorten. Allmählich begann durch die Ausbreitung der mesokratischen Hölzer in die bislang vorherrschenden Birken-KiefernBestände eine standörtliche Differenzierung innerhalb der Wälder. Zunächst ließ das Kronendach der Birken-Kiefern-Haselwälder genügend Licht auf die bodennahe Krautschicht durch, so dass sich dort Calluna und Filicinae ausbreiten konnten. Für die 
heliophilen Gattungen Artemisia und Filipendula waren die Lichtverhältnisse in der Krautschicht nicht mehr ausreichend.

Die Ufervegetation war von den Veränderungen in den Birken-Kiefern-Wäldern nicht betroffen und bildete weiterhin eine überwiegend aus Cyperaceae, Sparganium und Polypodiaceae zusammengesetzte Verlandungsgesellschaft.

\subsubsection{Atlantikum (VI, VII)}

Der Etablierung und langsamen Ausbreitung der Erle auf den feuchten und nassen Standorten, die bereits im ausklingenden Boreal eingesetzt hatte, folgte zu Beginn des Älteren Atlantikums, ca. 7.700 cal. B.C. deren Massenausbreitung, wie der steile Anstieg der Erlenkurve zu Beginn von W 3 belegt. Bevorzugte Standorte der Erle waren nicht nur die Uferbereiche des Sees, wo sie in Konkurrenz zur Hasel trat, sondern auch die umliegenden grundwassernahen und feuchten Standorte, von denen sie die Birke verdrängte.

Der kontinuierliche Rückgang der lichtliebenden Hasel im Älteren Atlantikum lässt sich außerdem auf die zunehmende Beschattung durch die erhöhten Bestandsanteile an Eichen und Ulmen zurückführen. Offensichtlich profitierte die Kiefer kurzfristig von dem Rückgang der Hasel im Unterholz (W 3, 1317-1097 cm), nahm aber wieder ab, als die Hasel und die Linde erneut an Bedeutung gewannen. Der Anteil der Linden an den atlantischen Wäldern dürfte höher gewesen sein, als die geringen Prozentwerte im Pollendiagramm vermuten lassen, da die Linde aufgrund ihrer geringen Pollenproduktion und ihrer Entomogamie im Pollendiagramm häufig deutlich unterrepräsentiert ist (LANG 1994). Vergleichbares gilt für die Esche. Es ist anzunehmen, dass die Esche trotz der niedrigen Werte, bereits seit dem Boreal im Untersuchungsgebiet anwesend war. In der Mitte des Älteren Atlantikums, ca. 6.400 cal. B.C., erreichte die Esche die empirische Pollengrenze. Ihre Ausbreitung, die kennzeichnend ist für den Beginn des Jüngeren Atlantikums (L PAZ W 4), erfolgte ca. 5.800 cal. B.C.. Die Esche bevorzugt laut OBERDORFER (1994) nährstoff- und basenreiche Ton- und Lehmböden, so dass sie in den bereits bestehenden linden- und ulmenreichen Eichenwäldern mit Hasel, Linde und Ulme um die favorisierten Standorte konkurrieren musste.

Die Ausbreitung der Linden, Ulmen und Eschen belegt eine Verbesserung der Standortbedingungen, möglicherweise hervorgerufen durch eine Zunahme der Niederschläge und daraus resultierend der Bodenfeuchte im Laufe des Atlantikums. Das regelmäßige 
Vorkommen von Hedera und Viscum lässt auf milde Winter und warme Sommer schließen (LANG 1994). Den veränderten edaphischen Bedingungen folgten weitere standörtliche Differenzierungen der Waldgesellschaften. Auf den mäßig feuchten, lehmigen Böden der kuppigen Grundmoränenlandschaft breiteten sich Laubmischwälder aus, in deren Strauchschicht die Hasel mit zunehmender Beschattung seltener wurde. Auf den trockenen, sandigen Standorten dominierte weiterhin die Kiefer zusammen mit der Birke. Bis zum Ende des Jüngeren Atlantikums blieb der Zustand der ,atlantischen“ Wälder relativ stabil.

\subsubsection{Subboreal (VIII)}

Während des Subboreals zeichneten sich erneut Veränderungen innerhalb der Wälder ab. Der Beginn des Subboreals (L PAZ W 5), der am Wustrowsee auf ca. 4.400 cal. B.C. datiert, wird durch den Rückgang der Ulmen und durch das Einsetzen der Fagus- und Plantago lanceolata-Kurve charakterisiert. Der Rückgang der Ulmen, der nach PERRY \& MOORE (1987) und Peglar \& BIRKs (1993) auf eine durch den Ulmensplintkäfer (Scolytus scolytus, S. multistriatus) übertragene Pilzerkrankung durch einen Ascomyceten (Ceratocystis ulmi) zurückzuführen ist, wurde wahrscheinlich zusätzlich durch klimatische Veränderungen und durch anthropogene Nutzung in Form von Schneitelung gefördert. Da die Standortansprüche der Rotbuche, die seit Beginn des Subboreals im Untersuchungsgebiet anwesend war, denen der Ulme gleichen, stellte sie eine Konkurrentin für die von Pilzbefall geschwächte Ulme dar, so dass schließlich eine Kombination aus zunehmendem Konkurrenzdruck, Klimawandel, Pilzbefall und anthropogener Nutzung zu einem Rückgang der Ulmenbestände führte. Die Hainbuche, die etwas später als die Rotbuche einwanderte, breitete sich ab ca. 2.600 cal. B.C. (W 5b) im Umkreis des Wustrowsees aus. Durch die Ausbreitung der Rotbuchen und Hainbuchen erfolgte im Subboreal eine weitere Differenzierung der Laubwaldgesellschaften auf den nährstoffreichen, feuchten Grundmoränenstandorten. Außerdem führte der zunehmende menschliche Einfluss zu Veränderungen innerhalb der Waldgesellschaften (s. 6.1.4.1). Möglicherweise führte ein selektiver Einschlag von Kiefern in den Kiefern-Eichenmischwäldern zu einer indirekten Förderung der Laubhölzer, die infolge der Freistellung mit einer erhöhten Pollenproduktion reagierten (vgl. BEUG 1992; РОтT 2000), wie der Rückgang der Kiefern-Kurve und erhöhte Ulmen- und Linden- Werte in W 5a belegen. Des weiteren wurde nach SCAMONI (1988) mittels der Beschattung durch die Eichen die Verjüngung der Kiefern verhindert, was durch den Rückgang der Kiefern-Kurve belegt sein könnte. 
Nach Wolters (2002) und RALSKA-JASIEWiCZOWA, NALEPKA et al. (2003) kam es während des Subboreals zu einer Zunahme der Humidität, die u. a. die Ausdehnung der subborealen Erlenbestände begünstigte. Eine gesteigerte Pollenproduktion der Erlen in Folge von Stockholzwirtschaft ist auszuschließen, da eine regelrechte Niederwaldwirtschaft in der Bronzezeit noch nicht zu erwarten ist (vgl. POTт 1986; Ротт 1990).

Die Mistel (Viscum), die kalte Winter gut erträgt, aber an warme Sommer gebunden ist (IVERSEN 1944), verlor durch den Rückgang von Ulmus und Tilia zwei ihrer bevorzugten Wirtsbäume - entsprechend rückläufig zeigt sich die Kurve im Pollendiagramm zu Beginn von W 5a. Die sich ausbreitende Rotbuche wurde von der Mistel als Wirtsbaum gemieden, so dass die Verbreitung der Mistel im Subboreal infolge des abnehmenden kontinentalen Charakters des Klimas und infolge des Rückgangs der Wirtsbäume allmählich nachließ. Das Efeu, das an luftfeuchte Standorte mit mäßig kalten Wintern gebunden ist und nach IVERSEN (1944) und LANG (1994) ebenfalls als Klimaindikator interpretiert wird, kam weiterhin regelmäßig vor. Bis zum Ende des Subboreals erfolgte die Ausbreitung von Rotbuchen und Hainbuchen sehr zögerlich. Ihr maximaler Anteil an der Baumpollensumme im Pollendiagramm vom Wustrowsee blieb noch unter $5 \%$. Am Krebssee, dessen Pollendiagramm gegen Ende des Subboreals einsetzt, betrug der Anteil von Fagus und Carpinus bereits mehr als $5 \%$.

\subsubsection{Der menschliche Einfluss auf die Vegetation im Neolithikum und in der Bronzezeit}

Wie bereist oben erwähnt, verstärkte sich im Laufe des Subboreals der menschliche Einfluss auf die bislang überwiegend natürliche Waldentwicklung. Die erhöhten Anteile der sekundären Siedlungszeiger (Rumex, Plantago lanceolata) in den Diagrammabschnitten W 5b und W 5d sind als Indikatoren für die Zunahme von neolithischen bzw. frühbronzezeitlichen Siedlungsaktivitäten in der Nähe des Wustrowsees zu interpretieren. Der Rückgang der Eichen und Linden in W 5b ist demnach als Folge von Rodungen zur Anlage von Siedlungsplätzen zu verstehen, da beide Baumarten, insbesondere die Linde, laut OBERDORFER (1994) frische bis mäßig trockene, humose, meist tiefgründige Lehmund Tonböden bevorzugen, die ebenso hervorragend für die Anlage von Siedlungsplätzen geeignet waren. Zusätzlich wurden die Linden zur Gewinnung von Laubheu für die Fütterung im Winter geschneitelt (AABY 1986; HAAS \& RASMUSSEN 1993). Eine weitere Nutzung erfolgte durch das Vieh, das zur Waldweide in den Wald getrieben wurde. Auf diese Weise kam es zu Auflichtungen in den vorherrschenden Eichenmischwäldern und in 
Folge des erhöhten Lichtgenusses in Bodennähe zur Ausbildung einer Krautschicht, wie der Anstieg der Poaceae dokumentiert. Der Anbau von Getreide ist durch den Nachweis vom Triticum-Typ belegt. Die Ackerflächen müssen in Seenähe gelegen haben, da die meisten Getreidearten (mit Ausnahme von Secale) aufgrund ihrer Kleistogamie kaum Pollen ausstreuen und nach BEHRE \& KUČAN (1986) der Nachweis von Getreide-Pollen mit zunehmender Entfernung der Ackerflächen von der Entnahmestelle nachlässt. Diese Siedlungsphase (W 5b) lässt sich archäologischen Befunden zufolge der Trichterbecherkultur zuordnen. Mit dem Abschnitt W 5c folgte eine Phase nachlassender Siedlungstätigkeit, wie an dem Rückgang der zuvor vermehrt vorkommenden Kulturpflanzen und sekundären Siedlungszeiger und an dem Wiederanstieg der Eichen abzulesen ist.

In der Frühbronzezeit ab 1.900 cal. B.C. wurde die Siedlungstätigkeit im Umkreis des Wustrowsees wieder aufgenommen (siehe W 5d). Während dieser Siedlungsphase, die der Urnenfelderzeit zugeordnet wird, breiteten sich Bevölkerungsgruppen der Lausitzer Kultur im Untersuchungsgebiet aus. Dem Ackerbau kam während dieser Zeit, den sporadischen Getreidenachweisen zufolge, nur eine unbedeutende Rolle zu, während die Waldweide vermutlich eine größere Rolle spielte, wie an den erhöhten Poaceae- und Calluna-Werten $\mathrm{zu}$ erkennen ist (BEHRE \& KUČAN 1986). Außerdem weisen die erhöhten Anteile von Chenopodiaceae, Artemisia und Rumex auf eine zunehmende Ruderalisierung hin (BEHRE 1981). Den archäologischen Befunden zufolge war das östliche untere Odertal während der Bronzezeit dicht besiedelt (siehe Kapitel 3.3), so dass eine erhebliche Auflichtung der Wälder erfolgt sein muss. Der Anstieg der Grünalgen belegt die zunehmende Eutrophierung des Wustrowsees.

\subsection{5 Älteres Subatlantikum (IX)}

Der Beginn des Älteren Subatlantikums, der durch den endgültigen Rückgang der HaselWerte auf weniger als $10 \%$ und den gleichzeitigen Rückgang von Tilia gekennzeichnet ist, wurde am Wustrowsee auf ca. 1.100 cal. B.C. datiert. Am Krebssee, wo der Übergang VIII/IX mit ca. 900 cal. B.C. datiert wurde, reagierte allerdings nur die Linde mit einem Rückgang.

Durch die zunehmende Verbreitung der Hainbuchen und durch den wachsenden menschlichen Einfluss kam es im Älteren Subatlantikum zu einer erneuten Differenzierung der Waldgesellschaften. Unter dem dichten Kronendach der Laubmischwälder, in denen der Anteil an Linden und Ulmen immer geringer wurde und in die allmählich die Rotbuchen 
und die Hainbuchen vorstießen, konnte die lichtliebende Hasel nicht mehr gedeihen, so dass sich ihr Vorkommen im Laufe des Älteren und Jüngeren Subatlantikums auf die lichten Waldränder bzw. auf die Seeufer-Standorte beschränkte.

Ein Rückgang der Kiefer, der auch von anderen, innerhalb des pommerschen Stadiums der Weichselvereisung liegenden Untersuchungspunkten bekannt ist (vgl. MüLLER 1969; JAHNS 2000), wird von MÜLLER (1969) auf eine Zunahme der Humidität zurückgeführt, die u. a. die Ausbreitung der Laubhölzer begünstigte. Dies wird durch die Ausbreitung der Rotbuche ab 500 cal. B.C. belegt. MüLLER (1969) räumt jedoch ein, dass die Kiefer lediglich von den nährstoffreichen Grundmoränenstandorten durch die Laubhölzer verdrängt wurde, während sie auf den nährstoffärmeren Standorten (Talsande) weiterhin dominierte. Die Datierungen des Kiefernrückgangs am Wustrowsee (W 6c; ca. 350 cal. B.C.) und am Krebssee (K 2a; ca. 400 cal. B.C.) zeigen eine sehr gute Übereinstimmung mit den Angaben von JAHNS (2000) aus westlich angrenzenden Untersuchungsgebieten und korrelieren mit der in BROSE (2002) beschriebenen Aufhöhung der Auensedimente infolge einer erhöhten Wasserführung während der vorrömischen Eisenzeit.

Der Rückgang der heliophilen Birken ist mit hoher Wahrscheinlichkeit ebenfalls auf die zunehmende Beschattung durch die Rotbuchen und Hainbuchen zurückzuführen. Die Erlenbestände hingegen waren von dem Konkurrenzdruck und der Zunahme der Humidität kaum betroffen, da sie aufgrund ihrer Unempfindlichkeit gegenüber Staunässe die für die übrigen Laubwaldarten ungeeigneten bodenfeuchteren bis vernässten Standorte einnehmen konnten.

\subsubsection{Der menschliche Einfluss auf die Vegetation von der Eisenzeit bis ins slawische Mittelalter}

Der Beginn des Älteren Subatlantikums, der gleichzeitig den Beginn der Eisenzeit darstellt, korreliert am Wustrowsee mit einem Nachlassen der Siedlungstätigkeit, wie der Rückgang von Poaceae, Artemisia, Plantago lanceolata und Rumex in W 6a und W 6b belegt. Damit bestätigen die pollenanalytischen Untersuchungen den von LEWCZUK (2002) angenommenen Besiedlungsrückgang bzw. die ausgeprägte Besiedlungsdiskontinuität östlich des unteren Oderlaufs während der vorrömischen Eisenzeit, die nach BROSE \& HEUßNER (2002) wahrscheinlich auf den Anstieg des Wasserspiegels zurückzuführen ist. Für die zuvor stark aufgelichteten Wälder begann mit dem Nachlassen der Besiedlung eine Regenerationsphase. Zunächst breiteten sich Birken auf den ehemals genutzten 
Flächen aus. Auch die Rotbuchen und Hainbuchen profitierten von dem nachlassenden Siedlungsdruck und dehnten ihre Bestände weiter aus, wie das erste Carpinus-Maximum in W $6 \mathrm{~b}$ belegt. Die Umgebung des Krebssees war zur gleichen Zeit offensichtlich weiterhin besiedelt, wie der Nachweis von Getreide und sekundären Siedlungszeigern belegt. Ab ca. 800 cal. B.C. zählte am Krebssee neben Weizen, Gerste und Hafer auch erstmals der Roggen zu den Kulturpflanzen. Allerdings ist der Roggen zu dieser Zeit noch als Segetalart zu interpretieren, denn nach BEHRE (1992) ist eine Kultivierung des Roggens in Polen erst ab der römischen Kaiserzeit zu erwarten. Auf das Bestehen von Weideflächen verweisen Poaceae und Plantago lanceolata. Die Siedlungsaktivitäten lassen sich vermutlich auf Bevölkerungsgruppen der Jastorf-Kultur zurückführen. Ab ca. 400 cal. B.C. zeichnet sich am Wustrowsee eine Zunahme des menschlichen Einflusses ab. Dies ergibt sich einerseits aus dem regelmäßigen Nachweis von Getreidepollen in W 6c, andererseits aus dem abrupten Rückgang der Kiefern, Rotbuchen und Hainbuchen, der vermutlich auf Rodungen zurückzuführen ist. Der ab ca. 300 cal. B.C. auftretende Roggen kam vermutlich als Begleitart von Wintergetreide vor. Polygonum aviculare, Plantago major/media und Chenopodiaceae belegen eine Zunahme der Ruderal- und Trittgesellschaften (BEHRE 1981). Es ist denkbar, dass der Anstieg des Wasserspiegels zu einer Verlagerung der Besiedlung von den Auenstandorten $\mathrm{zu}$ den höher gelegenen Grundmoränenkuppen führte, auf denen sich zuvor die Rotbuche etabliert hatte.

Die während der Völkerwanderungszeit erneut nachlassenden Siedlungsaktivitäten finden in den Pollendiagrammen vom Wustrowsee und Krebssee in den Abschnitten W 6d und K 2b ihre Entsprechung. Der Diagrammabschnitt K 2b dokumentiert die Wiederbewaldung aufgelassener Wirtschaftsflächen durch Betula in der Umgebung des Krebssees. Auf den nährstoffreichen mäßig feuchten Standorten dominierten bald strauch- und krautschichtarme Eichen-Hainbuchenwälder, wie an den geringen Hasel- und NBP-Werten abzulesen ist. Auf den feuchteren, nährstoffreichen Grundmoränenstandorten im Umkreis des Wustrowsees breiteten sich Rotbuchen aus.

Mit dem Beginn des slawischen Mittelalters kam es erneut zu anthropogenen Veränderungen der Wälder. Am Wustrowsee kam es infolge von Rodungen zu einem Rückgang von Fagus und Carpinus und somit zu einer Auflichtung der Wälder. Von den Auflichtungen profitierten einerseits die Eichen, die vermutlich wegen ihrer Nutzung als Mastbäume zunächst von Rodungen verschont blieben und andererseits die Ulmen, deren Blühfähigkeit und Pollenproduktion infolge der Freistellung zunahm, wie an dem Anstieg der 
Kurven der beiden Arten in W 6e abzulesen ist. Die Zunahme von Kulturpflanzen, Poaceae, Plantago lanceolata und Rumex, belegt Siedlungsaktivitäten wie Ackerbau und Weidewirtschaft. Gleichzeitig entwickelten sich in Siedlungsnähe Trittgesellschaften, wie der Nachweis von Chenopodiaceae und Polygonum aviculare dokumentiert. Die gegenüber den geringen Nachweisen von Weizen und Hafer erheblich höheren Anteile von Roggen, dessen Kultivierung nach BEHRE (1992) seit der römischen Kaiserzeit anzunehmen ist, lassen auf einen Wintergetreideanbau schließen.

Am Krebssee waren in der ersten Phase der slawischen Besiedlung (K 2 c) vor allem die Hainbuchen von Rodungen betroffen. Fagus erreichte zu dieser Zeit am Krebssee sogar Maximalwerte. Ab ca. 760 cal. A. D. wurden auch Rotbuchen gerodet. Neben dem Ackerbau nahm die Grünlandwirtschaft eine bedeutende Stellung ein, wie die deutlich erhöhten Anteile von Poaceae, Cyperaceae, Plantago lanceolata und Calluna zeigen. In Folge der intensiven Bewirtschaftung kam es, wie am Wustrowsee, zur Ausbreitung von Unkrautgesellschaften, wie nach BEHRE (1981) das vermehrte Vorkommen von Polygonum aviculare, Chenopodiaceae und einiger Brassicaceae belegt.

\subsubsection{Jüngeres Subatlantikum $(X)$}

Der Übergang vom Älteren zum Jüngeren Subatlantikum wird durch den deutlichen Anstieg des NBP in Folge der Zunahme der mittelalterlichen und neuzeitlichen Besiedlung angezeigt. Am Wustrowsee datiert dieser Übergang nach Extrapolation auf ca. 1.050 cal. A. D., für das Gebiet um den Krebssee wurde ein Alter von ca. 1.270 cal. A. D. errechnet.

Die stark aufgelichteten Laubwälder im Umkreis des Wustrowsees und Krebssees wurden weiterhin intensiv genutzt. Infolge der Neugründungen deutscher Siedlungen bestand ein enormer Holzbedarf, so dass durch Rodungen die Waldbestände deutlich degradiert wurden. Besonders betroffen waren davon Fagus, Carpinus und Quercus. Wie an dem Rückgang der Erlen abzulesen ist, wurden erstmals auch die feuchten Niederungen, vermutlich als Weideland, landwirtschaftlich genutzt.

\subsubsection{Der menschliche Einfluss auf die Vegetation im Mittelalter und in der Neuzeit}

Die Zunahme der Besiedlung während des deutschen Mittelalters und die damit einhergehenden Entwaldungen widerspiegeln sich besonders eindrucksvoll im Summendiagramm vom Krebssee in dem zugunsten des NBP verschobenen BP-NBP-Verhältnis des Diagrammabschnitts K 3a. Demnach war die Umgebung des Krebssees vermutlich zum 
großen Teil entwaldet, wie der extrem hohe Anteil an NBP und die extrem geringe Pollenkonzentration belegen. Die durch die Entwaldung geförderte Erosion führte zu einem erhöhten Eintrag in den See, infolge dessen die Eutrophierung voran schritt. Dies belegt die Ausbreitung von Grünalgen. Außer dem Getreideanbau, der neben Weizen und Gerste vor allem den Anbau des autogamen Roggens umfasste, ist eine Kultivierung von Hanf anzunehmen. Hafer wurde dagegen nur in geringen Mengen angebaut.

Die Ansprache des Hanfs wurde anhand der Auswertung der Größenmessungen des Humulus/Cannabis-Typs, die eine Häufung von Pollenkörnern mit einem Durchmesser größer als $26 \mu \mathrm{m}$ ergab und nach SMETTAN (1989) und DöRFLER (1990) eindeutig als Cannabis (Hanf) anzusprechen sind, abgesichert. Als weitere Ölpflanze kommt Leindotter (Camelina sativa) in Frage. Diese zur Familie der Brassicaceae gehörende Ölpflanze, deren Anbau anhand von Makroresten nach KNÖRZER (1978) und KÖRBER-GROHNE (1995) bereits aus der Bronzezeit bekannt ist, kann pollenanalytisch nicht nachgewiesen werden, da eine Unterscheidung von anderen Brassicaceae-Pollentypen nicht möglich ist. Die erhöhten Brassicaceae-Werte könnten jedoch zum Teil auf den Anbau von Leindotter zurïck geführt werden. Die verstärkte Ausbreitung von Urtica, Polygonum aviculare, Chenopodiaceae und verschiedenen Asteraceae in den Ruderal- und Trittgesellschaften ist als eine Folge der intensiven anthropogenen Nutzung zu werten. Als typische Segetalart in Roggenfeldern tritt Centaurea cyanus seit Beginn des intensiven Roggenanbaus vermehrt auf (BEHRE 1992). Der Roggen, dessen Aussaat überwiegend im Herbst erfolgte, gewann im Laufe des Mittelalters zunehmend an Bedeutung und entwickelte sich schnell zur wichtigsten Anbaufrucht unter den Getreidearten, da er vor allem auf den sandigen Böden höhere Erträge erbrachte, als die übrigen Getreidearten (WILLERDING 1986a; BEHRE 1992).

Für die Umgebung des Wustrowsees wird zur Zeit des deutschen Mittelalters ebenfalls ein deutlicher Rückgang der Waldbedeckung angenommen. Obwohl das Pollendiagramm eine Zunahme der Eichenbestände suggeriert, belegt der Polleninflux eindeutig den Rückgang aller Laubholzarten. Der Anstieg der Eichen-Kurve ist daher nur als rechnerisches Ergebnis zu bewerten. Der Anstieg der Kiefernkurve ist dagegen in der Tat als eine Ausdehnung der Kiefernbestände zu interpretieren. Die Zusammensetzung der Gruppe der Siedlungszeiger am Wustrowsee entspricht im wesentlichen derjenigen vom Krebssee, jedoch wird bei weitem nicht die gleiche Quantität erreicht. Dies mag daran liegen, dass einerseits das Gebiet um den Wustrowsee noch stärker bewaldet war als das Gebiet um 
den Krebssee und andererseits die Wirtschaftsflächen in größerer Entfernung vom See lagen und daher die Siedlungszeiger im Pollendiagramm weniger stark repräsentiert sind (vgl. BEHRE \& KUČAN 1986).

Im Pollendiagramm vom Krebssee dokumentieren der Rückgang der Kulturpflanzen ab $25 \mathrm{~cm}$ und der gleichzeitige Wiederanstieg von Pinus und Carpinus, der auf ca. 1.660 cal. A.D. datiert, die Auswirkungen der hochmittelalterlichen Wüstungsperiode und wahrscheinlich diejenigen des 30-jährigen Krieges. Trotz der Zunahme von Pinus und Carpinus blieb die Umgebung des Krebssees weitestgehend unbewaldet, wie der weiterhin hohe Anteil des NBP zeigt. Während der Neuzeit (K 3b) kam es u. a. durch die Maßnahmen einer geregelten Waldnutzung, veranlasst durch die Landesherren, zu einer vorübergehenden Waldregeneration. Um den weiterhin hohen Holzbedarf decken zu können, setzte laut FIRBAS (1952) ab dem 18. Jahrhundert die Aufforstung reiner Kiefernwälder ein, was sich im Pollendiagramm durch den Anstieg der Kiefern-Kurve in K 3b widerspiegelt. Der Rückgang der Bewaldung findet seine Bestätigung ebenso in dendrochronologischen Auswertungen von Holzproben aus archäologischen Ausgrabungen aus dem östlichen Brandenburg. Demnach fällt nach dem 14. Jahrhundert das Alter der Bauhölzer drastisch ab (HEUßNER 2002). In den südlichen Gebieten Brandenburgs bestand das Bauholz erwartungsgemäß zum überwiegenden Teil aus Kiefernholz, während im nordöstlichen Brandenburg sowie in großen Teilen Mecklenburgs hauptsächlich Eichenholz Verwendung fand (HEUßNER 2002).

\subsection{Die Vegetationsentwicklung im Umkreis von Rybojady und Pawski Lug}

\subsubsection{Das Problem der Ansprache des Spätglazials im Pollendiagramm von Rybojady}

Die Zuordnung des untersten Diagrammabschnitts $\mathrm{R} 1 \mathrm{zu}$ einem der spätglazialen Vegetationsabschnitte stellt sich als problematisch heraus. Die den Diagrammabschnitt R 1 charakterisierenden erhöhten Anteile von Hippophae, Salix und Helianthemum werden in polnischen Spätglazialprofilen entweder der Älteren Dryas im Sinne von MANGERUD et al.(1974) zugeordnet (vgl. TOBOLSKI \& OKUNIEWSKA-NOvACZYK 1989) oder nach LITT (1988) der Ältesten Dryas im Sinne von IVERSEN (1954). Überträgt man dieses auf das Profil von Rybojady, stellt man fest, dass die chronostratigraphisch jüngeren spätglazialen Vegetationsabschnitte Alleröd und Jüngere Dryas pollenstratigraphisch nicht eindeutig zu identifizieren sind. 
Der an R 1 angrenzende Diagrammabschnitt R 2 dokumentiert jedoch ohne Zweifel den Beginn der postglazialen, holozänen Vegetationsentwicklung und ist somit dem Präboreal zuzuordnen. Sedimentstratigraphisch gibt es keine Anzeichen für einen Hiatus, der das Fehlen von Bölling, Alleröd und Jüngere Dryas erklären würde.

Da der Beginn des Postglazials im Diagrammabschnitt R 2 erfasst ist, müsste also der Abschnitt R 1 dem Spätglazial, der Jüngeren Dryas, zugeordnet werden. Da sich die Jüngere Dryas in Spätglazialprofilen in der Regel durch einen hohen Anteil von Juniperus und Artemisia auszeichnet, wie u.a. den Arbeiten von LITT (1988), BRAndE (1980), MileckA (1991) und BitTMAnN \& PASDA (1999) zu entnehmen ist, aber im R 1 weder Juniperus noch erhöhte Artemisia-Werte vorliegen, ist nach den biostratigraphischen Kriterien eine Ansprache der Jüngeren Dryas ebenfalls nicht möglich. Ein ähnliches Problem zeigt sich in einem von JAHNS (2000) untersuchten Profil im östlichen Brandenburg. Hier zeigen die Pollenspektren des ausgehenden Spätglazials eine mit Rybojady vergleichbare Zusammensetzung. Durch den Nachweis von Laacher Seetephra wurde die Ansprache des Alleröds jedoch abgesichert. Das Fehlen der Jüngeren Dryas führt JAHNS (2000) auf eine möglicherweise geringe Sedimentationsrate bzw. auf einen Hiatus zurück.

Auf der Basis der vorliegenden Untersuchungen lässt sich der Diagrammabschnitt R 1 nicht zufriedenstellend einem spätglazialen Vegetationsabschnitt zuordnen. Das Ergebnis der AMS-Datierung ergab ein Alter von 18.850 \pm 110 B.P.. Da die basalen Sedimente jedoch aufgrund von Umlagerungsprozessen einen hohen Anteil von allochthonen Pollenkörnern aufweisen, wird dieses Alter als unzuverlässig bewertet.

\subsubsection{Präboreal (IV)}

Der Beginn des Präboreals (R 2), der durch die zunehmende Erwärmung und der damit einher gehenden Einwanderung der mesokratischen Gehölze aus ihren Glazialrefugien gekennzeichnet ist, wurde im Profil von Rybojady nach Extrapolation mit ca. 11.600 cal. B.C. datiert.

Lichte Birken-Kiefern-Wälder mit einer relativ gut entwickelten Krautschicht herrschten vor, wie die anfangs noch erhöhten Poaceae und Artemisia-Werte dokumentieren. Mit der Einwanderung von Corylus und Ulmus, die beide ihre empirische Pollengrenze bereits mit dem Beginn des Präboreals erreicht hatten, verdichteten sich die Wälder, so dass der Lichtgenuss für eine gut entwickelte Krautschicht nicht mehr ausreichte. Aufgrund ihrer Zoochorie verbreitete sich die Hasel sehr schnell. Ihre Anwesenheit im Umkreis vom 
Rybojady-See ist ab ca. 10.500 cal. B.C. belegt. Etwa 400 Jahre später war auch die Ulme im Gebiet vorhanden, wie laut HUNTLEY \& BIRKS (1983) das Erreichen von Werten > $2 \%$ belegt.

Hinweise auf einen präborealen Klimarückschlag, der nach BRANDE (1980a), ILLIG \& LANGE (1992) und BITTMANN \& PASDA (1999) in Brandenburg vor der Einwanderung der thermophilen Gehölze einsetzte und durch einen Birkenvorstoß und Kiefernrückgang gekennzeichnet ist, liefert das Pollendiagramm vom Rybojady-See aufgrund des zu großen Probenabstandes und der daraus resultierenden geringen zeitlichen Auflösung nicht.

\subsubsection{Boreal (V)}

Kennzeichnend für den Übergang vom Präboreal zum Boreal ist die rasche Ausbreitung der Hasel in Mitteleuropa (LANG 1994). Demnach lässt sich der Beginn des Boreals aufgrund des steilen Anstiegs der Corylus-Kurve im Diagrammabschnitt R 3 des Pollendiagramms von Rybojady durch Extrapolation auf etwa 9.600 cal. B.C. datieren. Die bevorzugten Standorte der lichtliebenden Hasel lagen in erster Linie im Bereich des Seeufers, außerdem breitete sie sich auch in den borealen Birken-Kiefern-Wäldern aus. Aufgrund der zunehmenden Verdichtung der Strauchschicht durch die Hasel reichte der Lichtgenuss für eine ausgeprägte Krautschicht nicht mehr aus - dies belegt der Rückgang der lichtbedürftigen Taxa Artemisia und Filipendula. Statt dessen etablierten sich weniger lichtbedürftige Arten wie Calluna und Farne (Filicinae).

Mit dem Beginn des Boreals kam es zur verstärkten Ausbreitung der Ulmen, der ab ca. 8.200 cal. B.C. die Ausbreitung der Eiche folgte. Damit setzte eine sukzessive Umwandlung der borealen Birken-Kiefern-Wälder in standörtlich differenzierte KiefernEichenmischwälder ein. Die lichtliebende Birke wurde unter dem Konkurrenzdruck der Eichen auf die nährstoffärmeren Standorte verdrängt. Auf den feuchten bis nassen, grundwassernahen Standorten etablierte sich die Erle (vermutlich Schwarzerle), deren Ausbreitung seit Beginn des Boreals langsam fortschritt.

\subsubsection{Atlantikum (VI, VII)}

Die Kriterien für den Beginn des Älteren Atlantikums (VI) werden in dem Diagrammabschnitt R 4 erfasst. Den AMS-Datierungen zufolge erfolgte der rapide Anstieg der Erlen, der den Beginn des Atlantikums kennzeichnet, ca. 7.000 cal. B.C.. Gleichzeitig hatte die Linde das Gebiet um den Rybojady-See erreicht und drängte ebenfalls auf die nährstoff- 
reichen, mäßig feuchten Standorte. Dies führte zu einer interspezifischen Konkurrenz unter den Eichenmischwaldarten und zu einer weiteren Differenzierung der bereits im Boreal begonnenen Umstrukturierung der Wälder. Es entwickelten sich kiefernreiche Mischwälder, wahrscheinlich mit einer gut entwickelten Strauchschicht, in der u.a. Haseln vorkamen. Infolge der Ulmen-, Eichen- und Lindenausbreitung nahm die Beschattung der Strauch- und Krautschicht zu, so dass der Anteil der lichtliebenden Hasel im Unterwuchs sowie der Anteil der Gräser in der Krautschicht im Laufe des Atlantikums abnahmen. Das Vorkommen von Haselsträuchern beschränkte sich danach auf die Seeufer und die natürlichen Waldränder. Durch die zunehmende Beschattung wurde nach SCAMONI (1988) auch die Verjüngung der Kiefernwälder eingeschränkt. Auf den nährstoffreichen Standorten entwickelten sich je nach Bodenfeuchte verschiedene Varianten des Eichenmischwaldes. Auf den feuchteren Böden herrschten Ulmen vor, auf den trockeneren Standorten dominierten Eichen und Linden. Der Anteil der Linden war wahrscheinlich höher als die geringen Prozentwerte im Pollendiagramm vermuten lassen, da die Linde wegen ihrer effizienten zoogamen Pollenverbreitung nur geringe Mengen Pollen produziert und damit im Pollendiagramm deutlich unterrepräsentiert ist (LANG 1994). Auch die Esche produziert vergleichsweise wenig Pollen, so dass trotz der geringen Prozentwerte und der noch lückenhaften Präsenz im Pollendiagramm mit dem Vorkommen von Eschen zu rechnen ist.

Der Übergang vom Älteren zum Jüngeren Atlantikum (VII) ist nach FIRBAS (1949) durch einen deutlichen Anstieg der Fraxinus-Werte gekennzeichnet. Am Rybojady-See spiegelt sich diese Entwicklung zu Beginn des Diagrammabschnitts R 5 wider und datiert ca. 6.200 cal. B.C.. Das Pollendiagramm vom Pawski Ług erfasst mit seinem nur zwei Pollenspektren umfassenden Diagrammabschnitt P 1 gerade noch das Ende des Älteren Atlantikums. Die daran anschließende PAZ umfasst das Jüngere Atlantikum und beginnt nach Extrapolation um ca. 4.030 cal. B.C..

Während des gesamten Atlantikums, das in der Literatur als Phase des holozänen Klimaoptimums bezeichnet wird (HuNTLEY \& PRENTICE 1996), waren durch den Anstieg der Temperatur und durch die durchschnittlich höheren Niederschläge optimale Wachstumsbedingungen für die thermophilen Gehölze gegeben, so dass mit Ausnahme extremer Standorte die Ausbreitung der Eichenmischwaldarten voran schritt. Die Ausbreitung von Hedera und Viscum, deren Anwesenheit seit Beginn des Atlantikums im Untersuchungsgebiet belegt ist und die nach Troels-SMith (1960) und IVERSEN (1944) als Klimaindika- 
toren für milde Winter und warme Sommer gewertet werden können, bestätigt die klimatische Gunstphase, die auch während des Jüngeren Atlantikums weiterhin anhielt. Durch die Etablierung der Esche, die laut OBERDORFER (1994) die mäßig feuchten bis nassen, nährstoff- und basenreichen humosen Tonböden bevorzugt, schritt die standörtliche Differenzierung der Wälder fort. Reine Kiefernbestände entwickelten sich auf den laubholzfeindlichen Standorten wie z. B. auf den trockenen sandigen Böden, die in der Umgebung vom Rybojady-See reichlich vorhanden waren. Diese sind nach MATUSZKIEWICZ (1984) vor allem auf den höher gelegenen Terrassen des unteren Warta- und NotećTals zu erwarten. Des weiteren kommt die Kiefer nach OBERDORFER (1994) auch auf mäßig sauren, humosen Lehmböden sowie auf Torf vor.

\subsubsection{Subboreal (VIII)}

Das Subboreal, dessen Beginn durch den Rückgang der Ulmenwerte („Ulmenfall“) gekennzeichnet ist und im Pollendiagramm von Rybojady durch die Diagrammabschnitte R 6a - R 6c wiedergegeben wird, beginnt ca. 3.900 cal. B.C.. Im Pollendiagramm von Pawski Ług spiegeln die Abschnitte P $3 \mathrm{a}$ und P $3 \mathrm{~b}$ die Vegetationsentwicklung des Subboreals wider. Der Beginn von P 3a wurde mit ca. 3.700 cal. B.C. datiert.

Die bis zum Subboreal im wesentlichen durch natürliche Faktoren determinierte Waldentwicklung wurde im Laufe des Subboreals zunehmend durch menschliche Aktivitäten beeinflusst. Im Umkreis von Rybojady ist seit Beginn des Subboreals mit der Anwesenheit von Fagus zu rechnen. Die Fagus-Einwanderung in das Gebiet um das Pawski Ług wurde anhand des Beginns der geschlossenen Fagus-Kurve mit ca. 3.500 cal. B.C. datiert. Etwa gleichzeitig mit der Rotbuche drang auch die Hainbuche in die bestehenden KiefernEichenmischwälder ein. Sie bevorzugt nach OBERDORFER (1994) frische bis mäßig trockene, mäßig nährstoffreiche Sand- und Lehmböden. Wegen ihrer Unempfindlichkeit gegenüber Spätfrösten tritt sie in den Gebieten, die durch ein kontinentales Klima geprägt sind, gegenüber der spätfrostempfindlichen Rotbuche stärker hervor (vgl. ELLENBERG 1996). Der Rückgang von Hedera und Viscum seit der Mitte des Subboreals liefert Hinweise auf klimatische Veränderungen, da insbesondere Hedera nach IVERSEN (1944) mit abnehmender Ozeanität an Blühfähigkeit (= Rückgang der Pollenproduktion) einbüßt.

Die Ausbreitung von Fagus- und Carpinus, die durch das Erreichen der $1 \%$-Grenze gekennzeichnet ist, korreliert in beiden Pollendiagrammen mit dem vermehrten Auftreten von Siedlungszeigern, so dass neben den natürlichen Sukzessionsabläufen eine Bindung 
beider Arten an anthropogenen Standorten nicht auszuschließen ist. Dies wird von SCHLEICHER (2002) bestätigt, die in $90 \%$ der ihren Untersuchungen zur Einwanderung und Ausbreitung von Fagus und Carpinus in Deutschland zugrunde liegenden Lokalitäten einen Zusammenhang zwischen geschlossener Fagus- bzw. Carpinus-Kurve und dem Auftreten von Cerealia und Plantago lanceolata nachweisen konnte.

Die im Pollendiagramm von Pawski Ług bereits im Laufe des Jüngeren Subatlantikums zunehmenden und zu Beginn des Subboreals erhöhten Anteile der Sphagnum-Sporen belegen eine Veränderung der hydrologischen Bedingungen innerhalb der Hohlform. Der Anstieg von Amphitrema deutet zwar nach VAN GEEL (1978) auf eine Zunahme der Humidität, jedoch lässt die Zunahme von Braunmoosradizellen oberhalb von $620 \mathrm{~cm}$ eher auf einen sinkenden Wasserspiegel, also einen Rückgang der Humidität schließen, infolge dessen eine Verlandung der Hohlform, möglicherweise unter semiaquatischen Bedingungen, einsetzte. In der ufernahen Röhrichtzone scheint Sparganium häufig gewesen zu sein.

\subsubsection{Der menschliche Einfluss im Neolithikum und in der Bronzezeit}

Der Nachweis von Siedlungsphasen in Pollendiagrammen ist nur dann gewährleistet, wenn die Siedlungsplätze bzw. die dazugehörigen Wirtschaftsflächen in unmittelbarer Nähe der dem Pollendiagramm zugrunde liegenden Ablagerungsstätte lagen. Die Gründe dafür wurden bereits in Kapitel 6.1.4.1 erläutert.

Neolithische Siedlungstätigkeiten spiegeln sich im Pollendiagramm von Rybojady im Diagrammabschnitt R 5 durch die leicht erhöhten Werte von Poaceae, Calluna und Pteridium wider. Demnach ist die Waldweide in dieser in das Spätneolithikum zu stellenden Siedlungsphase als Wirtschaftsform belegt (BEHRE \& KUČAN 1986). Dem Ackerbau kam eine geringe Bedeutung zu, wie das Fehlen von Getreidepollen belegt. Lediglich die leicht erhöhten Werte von Rumex, der im Falle von Rumex acetosella nach BEHRE (1981) eine Bindung an Ackerbau zeigt, und die ebenfalls leicht erhöhten Plantago lanceolata-Werte, die auf die Beweidung von Brachflächen verweisen (BEHRE \& KUČAN 1986), sind Hinweise für Ackerbau.

Im Pollendiagramm vom Pawski Ług spiegeln sich neolithische Siedlungsaktivitäten in den PAZ P 3a und P 3b durch die erhöhten Anteile von Calluna und Pteridium wider, die eine nutzungsbedingte Auflichtung der Wälder, einerseits durch Brandrodung (POTT 1986), andererseits durch Waldweidewirtschaft, anzeigen. Zusätzlich ist aufgrund des 
Rückgangs von Ulmen und Linden eine Waldnutzung durch Schneitelung anzunehmen, da beide Arten nach Schneitelung mit einem verminderten Blühverhalten reagieren (AABY 1986). Des weiteren wird eine zunehmende Ruderalisierung durch den Anstieg von Artemisia, von Plantago lanceolata, vom Trifolium-Typ und von Chenopodiaceae angezeigt. Archäologischen Untersuchungen zufolge könnten die Siedlungstätigkeiten auf die Besiedlung durch Schnurkeramiker zurückzuführen sein (KULCZYCKALECIEJEWICZOWA 2002).

Im Pollendiagramm von Rybojady zeichnet sich mit dem Beginn der Bronzezeit durch den Anstieg von Siedlungszeigern ebenfalls eine zunehmende Siedlungstätigkeit ab (siehe R 6b). Eine Intensivierung bronzezeitlicher Siedlungsaktivitäten reflektiert das Pollenspektrum des Abschnitts R 6c. Der Rückgang der EMW-Arten zeigt Rodungen und eine intensive Nutzung der Wälder durch Waldweide an. Die daraus resultierende Auflichtung der Wälder findet ihre Bestätigung in dem gleichzeitigen Anstieg der Sedimentzuwachskurve (vgl. Abbildung 2), denn infolge der abnehmenden Bewaldung erfolgte eine Zunahme der Erosion.

Der Nachweis von Getreide beläuft sich auf vereinzelte Pollenkörner, so dass dem Ackerbau gegenüber der Weidewirtschaft noch immer eine unbedeutende Rolle zukam, oder die Entfernung der Wirtschaftsflächen zum Rybojady-See war zu groß, so dass Pollenkörner des autogamen Getreides von dem dazwischen liegenden Wald abgefangen wurden (BEHRE \& KUČAN 1986). Die Zunahme von Tritt- und Ruderalgesellschaften ist durch den Anstieg von Artemisia, Plantago lanceolata, Plantago major/media und Polygonum aviculare eindeutig belegt und deutet auf das Bestehen von Siedlungen in Seenähe.

\subsection{6 Älteres Subatlantikum (IX)}

Der Beginn des Älteren Subatlantikums wurde anhand des Rückgangs von Corylus im Profil von Rybojady sowie auch im Profil von Pawski Ług mit ca. 1.160 cal. B.C. datiert. Während des Älteren Subatlantikums, welches durch die Diagrammabschnitte R 7a bis R 7d sowie P 4a bis P 4c repräsentiert wird, schritt die Ausbreitung von Fagus nur sehr zögerlich voran, während Carpinus in der Umgebung von Rybojady zu einem ersten Maximum anstieg. HESMER (1933) nimmt für die Umgebung von Pawski Ług Buchenwälder mit einem hohen Anteil von Linden und einem geringen Anteil von Eichen an. Dies kann anhand der vorliegenden Untersuchungen trotz der geringen Fagus-Werte 
bestätigt werden; denn unter Berücksichtigung des extrem hohen Anteils von PinusPollenkörnern in der BP-Summe, der den prozentualen Anteil allen übrigen Gehölzpollens reduziert, ist der Anteil von Fagus-Pollen im Diagramm vom Pawski Ług tatsächlich höher als errechnet. Bei einer Kalkulation der BP-Summe exklusive Pinus ergaben sich Fagus-Werte bis zu $20 \%$, was jedoch immer noch vergleichsweise gering ist, wenn man bedenkt, dass die Rotbuche auf geeigneten Standorten wie z. B. im südwestlichen Harzvorland Baumpollenanteile von mehr als $50 \%$ erreichte (HERKING 1998). Das bedeutet, dass die Buchenwälder im Gebiet um Pawski Ług wie auch im unteren Odertal wahrscheinlich nur inselartig verbreitet waren. Sie herrschten auf den nährstoffreichen und feuchten Böden vor, während auf den ärmeren und trockeneren Standorten Eichenmischwälder vorherrschten. Der extrem hohe Anteil von Kiefern-Pollen stammt wahrscheinlich von auf dem Moor wachsenden Kiefern.

Die pollenanalytischen Befunde belegen für die Umgebung von Rybojady ebenfalls eine nur sehr geringe Verbreitung von Buchenwäldern und einen höheren Anteil von EichenHainbuchen-Wäldern.

Im Laufe des Älteren Subatlantikums zeichnete sich in der Umgebung von Rybojady durch den Rückgang von Schwimmblattgesellschaften (Nymphaea, Nuphar) und von Grünalgen und durch die zeitgleiche Zunahme von Röhrichtgesellschaften (Typha latifolia-Typ, Sparganium) eine Veränderung der hydrologischen Bedingungen ab. Dies wird durch die stratigraphischen Befunde, die einen Wechsel von dunkler Feindetritusmudde zu schwach zersetzter Grobdetritusmudde anzeigen, bestätigt. Offensichtlich spiegelt sich hier die von VAN GEEL \& RENSSEN (1998) für Nordwesteuropa angenommene Klimaänderung um 2.650 B.P. wider. An der Bohrstelle endete die limnische Phase etwa ab $700 \mathrm{cal}$. A. D.. Die fortschreitende Verlandung und Oligotrophierung, die möglicherweise durch einen sinkenden Wasserspiegel verursacht wurde, ist durch die Ausbreitung von Cyperaceae, Vaccinium, Menyanthes und Drosera belegt. Die zu Beginn der Verlandung noch erhöhten Anteile von Utricularia deuten auf das partielle Fortbestehen von Flachwasserbereichen. Mit fortschreitender Verlandung nahmen diese Flachwasserbereiche jedoch ab, wie der sukzessive Rückgang von Utricularia dokumentiert.

\subsubsection{Der menschliche Einfluss auf die Vegetation von der Eisenzeit bis zum slawischen Mittelalter}

Die Vegetationsentwicklung im Älteren Subatlantikum zeigt, dass die Wälder in viel stärkerem Maße von menschlichen Eingriffen betroffen waren als zuvor. Es zeichnet sich 
zwar in beiden Pollendiagrammen zu Beginn der Eisenzeit ein Nachlassen der Siedlungstätigkeiten und in Folge dessen eine Waldregenerationsphase ab, diese ist jedoch nur von kurzer Dauer. Die aufgelassenen Flächen wurden rasch von Birken eingenommen. Außerdem breiteten sich auf den nährstoffreichen Böden Fagus und Carpinus weiter aus (siehe PAZ R 7a, P 4a). Gegen Ende der Eisenzeit, zu Beginn der Römischen Kaiserzeit reflektieren die Pollenspektren der PAZ R 7b im Pollendiagramm von Rybojady das Einsetzen erneuter Siedlungstätigkeiten im Untersuchungsgebiet. Vermutlich waren es Bevölkerungsgruppen der Przeworsk-Kultur, die sich in der Nähe der Hohlform ansiedelten (vgl. LEWCZUK 2002: Abb. 126). Zu den nachgewiesenen Kulturpflanzen zählten vor allem Weizen und Roggen. Allerdings ist nach LANGE \& LIEBETRAU (1973) im nordostdeutschen Flachland erst ab dem slawischen Mittelalter mit dem Anbau von Roggen zur rechnen. Als Unkraut in Getreidefeldern verbreitete sich jedoch der Roggen in Europa bereits seit der späten Bronzezeit (BEHRE 1992), so dass die Roggen-Werte dieser späteisenzeitlichen Siedlungsphase wahrscheinlich von Unkrautroggen stammen. Einen Hinweis auf den Anbau weiterer Kulturpflanzen liefert die deutliche Zunahme der Brassicaceae, die möglicherweise den Anbau von Leindotter (Camelina sativa) belegt. Für NW-Deutschland ist der Anbau von Leindotter als Ölpflanze seit der frühen vorrömischen Eisenzeit an Hand von Makrorestfunden nachgewiesen (KUČAN 1986), pollenanalytisch lässt sich der Nachweis von Leindotter aus bereits genannten Gründen nicht eindeutig belegen. Den relativ hohen Anteilen der Poaceae und Cyperaceae zufolge, spielte die Grünlandbewirtschaftung eine große Rolle. Außerdem ist laut BEHRE (1981) die Herausbildung von Ruderalflächen durch die gestiegenen Werte von Chenopodiaceae, Artemisia und Polygonum aviculare eindeutig belegt.

Ein Nachlassen der Siedlungsaktivitäten erfolgte in der Völkerwanderungszeit, die im Pollendiagramm von Rybojady und von Pawski Ług durch die Abschnitte R 7c und P 4b erfasst ist. Während dieser Zeit fand eine Regeneration der zuvor durch Waldweide und Schneitelwirtschaft stark geschädigten Eichenmischwälder statt, wie an dem Anstieg von Ulmus, Tilia, Quercus, Fagus und Carpinus zu erkennen ist.

Während des slawischen Mittelalters (PAZ R 7d, P 4c) wurden die Siedlungsaktivitäten im Umkreis von Rybojady und Pawski Ług wieder aufgenommen, wie der Rückgang der Gehölzpollenkurven belegt. Erstmals waren auch Rotbuchen und Hainbuchen von Rodungen betroffen. Bemerkenswert ist zudem der Rückgang der Erlenbestände, der eine Ausdehnung der Wirtschaftsflächen auf die feuchten Standorte bzw. eine niederwaldartige 
Nutzung der Erlenbestände vermuten lässt. Gleichzeitig setzte die oben beschriebene Verlandung in unmittelbarer Umgebung des Bohrpunktes des Rybojady-Sees ein.

Der relativ hohe Anteil von Roggen-Pollenkörnern zu Beginn der Siedlungsphase ist nun eindeutig auf kultivierten Roggen zurückzuführen. Neben dem Ackerbau bekam auch die Viehhaltung eine größere Bedeutung, wie der Anstieg der Grünlandzeiger Rumex (acetosa), Plantago lanceolata und Poaceae belegt. Diese könnten nach WILLERDING (1986b) auch vermehrt in Hudewäldern aufgetreten sein. Hinweise auf Hudewälder liefert das Verhalten der Eichen-Kurve, die trotz intensiver Rodungsmaßnahmen keine wesentlichen Veränderungen anzeigt. Einerseits wurden Eichen von Rodungen verschont, da sie als Mastbäume genutzt wurden, andererseits wurde durch Waldauflichtung und Freistellung deren Blüten- bzw. Pollenproduktion erhöht, wie bereits BEUG (1992) in seinen Untersuchungen im Unteren Eichsfeld nachweisen konnte, so dass im Pollendiagramm der eigentliche Rückgang der Eichen zum Teil durch eine gesteigerte Pollenproduktion kompensiert wird.

Im Umkreis von Pawski Ług bauten die slawischen Siedler neben Gerste und Weizen vor allem auch Roggen an. Für die Viehwirtschaft war weiterhin feuchtes Grünland (Poaceae, Cyperaceae, Plantago lanceolata) von Bedeutung. Die Zunahme von Artemisia, Chenopodiaceae, Polygonum aviculare und verschiedener Asteraceae zeigt eine Ausbreitung der Ruderal- und Trittgesellschaften an. Nachdem zunächst nur Buchen und Hainbuchen von Rodungen betroffen waren, wurden im weiteren Verlauf des slawischen Mittelalters, trotz nachlassender Siedlungstätigkeiten auch Eichen geschlagen (PAZ P 4c). Eine Zunahme der Siedlungstätigkeiten zeigt sich erst wieder gegen Ende des Älteren Subatlantikums durch den erneuten Anstieg der Getreide-Werte. Bemerkenswert ist der deutliche Rückgang der Kiefer, der vermutlich auf die Ausdehnung der Nutzflächen auf ehemalige Kiefernstandorte zurückzuführen ist. Durch die Auflichtungen nimmt der Anteil der lichtliebenden Gehölze wie Birke und Hasel rasch wieder zu. Auch scheinen sich die Erlenbestände von der Niederwaldwirtschaft regeneriert zu haben, was an dem Anstieg der Erlenkurve abzuleiten ist. Der Nachweis von Adlerfarn und Calluna belegt, dass weiterhin das Vieh zur Weide in den Wald getrieben wurde. Hinweise auf eine Grünlandwirtschaft fehlen. Die nachgewiesenen Humulus/Cannabis-PK sind nach dem Größenvergleich $(<25 \mu \mathrm{m})$ als Hopfen anzusprechen, der sich vermutlich in den Auengesellschaften ausbreitete. 


\subsubsection{Jüngeres Subatlantikum (X)}

Der Beginn des Jüngeren Subatlantikums ist palynostratigraphisch durch den steilen Anstieg der NBP-Kurve gekennzeichnet (siehe PAZ R 8a, R 8b und P 5). Dieser Anstieg datiert im Pollendiagramm von Rybojady ca. 1.300 cal. A. D.. Im Diagramm von Pawski Ług wurde ein Alter von ca. 1.100 cal. A. D. extrapoliert.

Während des Jüngeren Subatlantikums wurde die Vegetationsentwicklung fast ausschließlich durch den menschlichen Einfluss bestimmt. Infolge des mittelalterlichen Landesausbaus war der Bedarf an Nutzflächen und der Bedarf an Holz als Konstruktions- und Brennholz enorm, so dass die bereits stark aufgelichteten Wälder durch Rodungen weiter dezimiert wurden. Dies kommt besonders durch die Verschiebung des BP/NBPVerhältnisses zu Gunsten des NBP zum Ausdruck. Aufgrund des hohen Anteils von NBP wird angenommen, dass im Umkreis von Rybojady große waldfreie Flächen bestanden haben müssen. Rotbuchen und Hainbuchen waren besonders von Rodungen betroffen, wie deren Kurvenverhalten in beiden Pollendiagrammen zeigt. Der Anteil des Eichenpollens blieb trotz der Rodungsmaßnahmen zunächst noch konstant (siehe P 5), da die Eichen in den parkartigen Hudewäldern von Rodungen verschont blieben. Aufgrund der Freistellung bildeten sie breitere Kronen mit einer größeren Anzahl von Blüten aus, wodurch der Eichenpollenniederschlag anstieg (vgl. BEUG 1992). Mit Beginn der Neuzeit wurden auch die Eichen gerodet (siehe R 8b). Die intensive landwirtschaftliche Nutzung führte zu einer Nährstoffverarmung der Böden. Die durch die großflächigen Entwaldungen geförderte Erosion des nährstoffreichen, humosen A-Horizonts, der sich in den Laubmischwäldern entwickelt hatte, trug ebenfalls zur Nährstoffverarmung der Böden bei.

Im Pollendiagramm vom Pawski Ług wurde nur der Beginn des Jüngeren Subatlantikums erfasst, aber auch in diesem kurzen Abschnitt spiegelt der Rückgang von Fagus und Carpinus zunehmende Siedlungsaktivitäten wider.

\subsubsection{Der menschliche Einfluss auf die Vegetation im Mittelalter und in der Neuzeit}

Im Zuge des mittelalterlichen Landesausbaus entwickelte sich eine Kulturlandschaft, in der es neben regelmäßig bestellten Ackerflächen auch ausgedehnte, als Viehweide dienende Hutungen gab (KIRK 1960). Als Kulturpflanzen wurden Gerste, Weizen, Hafer und vor allem Roggen, letzterer vermutlich als Wintergetreide, angebaut. Als typischer Begleiter des Winterroggens auf sauren Böden trat Centaurea cyanus im Hochmittelalter dazu (BEHRE 1981). Zu den Kulturpflanzen des Mittelalters zählte offensichtlich auch der 
Hanf. Außer den Hanfnachweisen fehlen allerdings Hinweise auf weitere Faser- und Ölpflanzen. Durch den deutlichen Anstieg von Poaceae, Plantago lancelata und Rumex ist eine Zunahme der Grünlandvegetation anzunehmen. Allerdings dürfte sich ein Teil der Grünlandzeiger auch auf den Brachflächen ausgebreitet haben (BEHRE 1981; WILLERDING 1986b). Brachflächen waren durch die besonders im Mittelalter weit verbreitete Dreifelderwirtschaft reichlich vorhanden. Auf den aufgelassenen Flächen breiteten sich Pionierhölzer, darunter vor allem die Birke und auch Calluna aus. Die erhöhten Anteile von Calluna und von Cyperaceae stammen zum Teil auch aus dem lokalen Pollenniederschlag der Mooroberflächenvegetation. Des weiteren ist laut BEHRE (1981) eine Ausbreitung von Calluna in den durch Waldweide genutzten Wäldern zu erwarten. Außerdem zeigen die erhöhten Anteile der Ericaceae eine zunehmende Oligotrophierung der Böden an. Die Zunahme von Ruderal- und Trittgesellschaften wird durch das vermehrte Auftreten der nitrophilen Urtica und Chenopodiaceae sowie durch Polygonum aviculare angezeigt.

Seit der Neuzeit wurde in der Umgebung von Rybojady Buchweizen als neue Mehlfrucht angebaut. In den Hintergrund rückte der Anbau von Hanf, dessen Pollen in den neuzeitlichen Spektren nicht mehr nachgewiesen wurden. 


\section{Der zeitliche Ablauf der holozänen Vegetationsentwicklung entlang des östlichen unteren Odertals und südlichen unteren Wartatals}

Heutzutage erlauben modernste, auf wenigen Milligramm Kohlenstoff beruhende Radiocarbondatierungen, die mit Hilfe der Dendrochronologie kalibriert werden, genaue Aussagen zum zeitlichen Ablauf der Vegetationsentwicklung. Darüber hinaus liefern die detaillierten Untersuchungen an grönländischen Eisbohrkernen (DANSGAARD et al. 1993) sowie an laminierten Sedimenten (GoSLAR et al. 1999; LITT \& STEBICH 1999) relativ genaue Erkenntnisse über die Dauer und die Vegetationsentwicklung insbesondere in den von IVERSEN (1954) als Dryas Ia - Bölling Ib - Dryas Ic - Alleröd II - Dryas III klimatostratigraphisch differenzierten spätglazialen Vegetationsabschnitten. Durch die Verknüpfung von Pollenanalysen mit einer Warvenchronologie am selben Profil kann jeder Pollenprobe ein jahrgenaues Warvenalter zugeordnet werden. Sehr aussagekräftige Ergebnisse liefern diesbezüglich die Profile des Meerfelder Maares und des Holzmaares aus der Eifel (NEGENDANK et al. 1990; STEBiCh 1999; KubitZ 2000). Weitere Untersuchungen liegen u. a. aus England (PEGLAR et al. 1984), aus Italien (ZoLITSCHKA \& NEGENDANK 1993), aus der Schweiz (LOTTER 1989) und aus dem in Zentralpolen gelegenem Lake Gościąż (RALSKA-JASIEWICZOWA \& VAN GEEL 1992) vor.

Demnach können mit Hilfe der Radiocarbonmethode, der Warvenchronologie und der Dendrochronologie die in regional und klimatisch unterschiedlichen Untersuchungsgebieten auftretenden Vegetationsentwicklungen chronologisch eingeordnet werden. Auf diese Weise entsteht ein immer genaueres Bild der Vegetationsentwicklung. Durch den chronologischen Vergleich von Pollendiagrammen mit unterschiedlichen „local pollen assemblage zones“ (L PAZ) lassen sich differenzierte Aussagen hinsichtlich der Einwanderung und Ausbreitung verschiedener Gehölze ableiten. Die Zuteilung der lokalen Pollenzonen (L PAZ) zu den waldgeschichtlichen Abschnitten (IV-X) erfolgte für das Untersuchungsgebiet nach den in FIRBAS (1949) aufgestellten biostratigraphischen Kriterien zur spät- und postglazialen Waldentwicklung. Die in der Literatur häufig als „Firbas-Zonen“ angesprochenen waldgeschichtlichen Abschnitte sind demnach als Biozonen und nicht als Chronozonen zu verstehen. Auf der Basis von insgesamt siebenundzwanzig Radiocarbondatierungen soll im folgenden der zeitliche Ablauf der Vegetationsentwicklung am Krebs- und Wustrowsee sowie am Pawski Ług und Rybojady dargestellt werden. Dabei werden die Datierungen der pollenanalytischen Arbeiten aus 
den Nachbargebieten vergleichend herangezogen und diskutiert. Alle Altersangaben sind in cal. B.C. bzw. in cal. A.D., also in Kalenderjahren, angegeben.

Die Tabelle 10 gibt einen Überblick über die Datierungen der waldgeschichtlichen Abschnitte. Zum Vergleich wurden Datierungen aus westlich und östlich angrenzenden Untersuchungsgebieten angefügt, die sich ebenfalls auf die waldgeschichtlichen Abschnitte von FIRBAS (1949) beziehen.

\subsection{Beginn des Holozäns}

Wie bereits in Kapitel 6.2.1 erläutert, konnte anhand der vorliegenden palynostratigraphischen Befunde eine Gliederung des spätglazialen Vegetationsablaufs im Rybojady nicht vorgenommen werden. Die vorliegende AMS-Datierung aus diesem Abschnitt wird wegen des hohen Anteils umgelagerten Pollens als wenig glaubwürdig erachtet. Der Beginn des Holozäns lässt sich jedoch nach den biostratigraphischen Kriterien (Anstieg von Pinus und folgende Ausbreitung von Corylus und Ulmus) eindeutig festlegen und wurde mittels Extrapolation mit ca. 11.320 cal. B.C. datiert. Am Wustrowsee wurde nach Extrapolation ein Alter von 11.075 cal. B.C. für die ältesten Sedimente ermittelt. Damit ergibt sich eine erhebliche Diskrepanz zu den Angaben von WOLTERS (2002), der den Beginn des Holozäns in der Döbritzer Heide auf 9.670 cal. B.C. datiert, sowie zu den Angaben von GosLAR et al. (1995), die den Übergang zum Holozän bei ca. 9.560 cal. B.C. annehmen. Diese große Abweichung resultiert einerseits aus der Extrapolation, die gleichmäßige Sedimentationsraten voraussetzt, die aber tatsächlich nicht gegeben sein müssen. Andererseits führt ein sogenanntes ${ }^{14}$ C-Plateau um 10.000 B.P. zu Ungenauigkeiten bei den Datierungsergebnissen. Innerhalb dieses Plateaus werden über einen Zeitraum von mehreren hundert Jahren konstante ${ }^{14} \mathrm{C}$-Alter ermittelt (LOTTER et al. 1992). Für die hier untersuchten Gebiete kann daher der genaue Zeitpunkt des Beginns des Holozäns anhand der vorliegenden Datierungen nicht bestimmt werden. Nach warvenchronologischen Untersuchungen beginnt das Holozän in Nordwestdeutschland zwischen 9.640 und 9.530 cal. B.C. (BRAUER et al. 2001), in Norddeutschland ca. 9.610 cal. B.C. (MERKT \& MÜLLER 1999) und in Mittelpolen zwischen 9.600 und 9.530 cal. B.C.. Die Befunde aus dem GRIP-Eiskern, die den Beginn des Holozäns auf 9.600 cal. B.C. festlegen (BJÖRCK et al. 1998), zeigen sehr gute Übereinstimmung mit den anderen Datierungen, so dass der Beginn des Holozäns um ca. 9.600 cal. B.C. als gesichert gilt. 
Der Zeitpunkt der für das Präboreal charakteristischen Corylus- und Ulmus-Einwanderung kann aufgrund der unzuverlässigen Datierungsergebnisse nicht angegeben werden. Am Wustrowsee wanderten beide Arten zur gleichen Zeit ein, wie der Beginn der geschlossenen Kurven andeutet. Für die Umgebung von Rybojady kann aufgrund des Anteils umgelagerten Pollens diesbezüglich keine eindeutige Aussage getroffen werden.

\subsection{Boreal}

Das Boreal ist nach FIRBAS (1952) durch die Ausbreitung der mesokratischen Gehölze, insbesondere durch die rasche Ausbreitung der Hasel, charakterisiert. Am Wustrowsee ergibt eine Datierung wenige $\mathrm{cm}$ unterhalb des Übergang IV/V ein Alter zwischen 9.110 und 8.740 cal. B.C.. Vom Profil Rybojady liegt aus dem Bereich des Übergangs IV/V keine Datierung vor. Durch Interpolation wurde ein Alter von ca. 9.630 cal. B.C. ermittelt, ein Wert, der dem Beginn des Holozäns entspricht und nicht zutreffen kann. Vermutlich war die Sedimentationsrate im Interpolationsbereich nicht konstant.

Die Hasel, die bereits kurz nach ihrer Einwanderung im Präboreal hohe Anteile aufwies, erreichte im Boreal rasch Maximalwerte. Am Wustrowsee begann die Massenausbreitung der Hasel ca. 8.650 cal. B.C.. Etwa 600 Jahre später wurde ein erstes Maximum (135 \%) erreicht, dem weitere 200 Jahre später gegen 7.850 cal. B.C. ein zweites Maximum mit Werten um $230 \%$ folgte. Damit übertrifft die Hasel am Wustrowsee bei weitem die Werte der westlich der Oder gelegenen Nachbarregionen, die für das östliche Brandenburg, und zwar den Großen Krebssee, mit etwa knapp 70 \% (JAHNS et al. 2002) und für den Berliner Raum nur mit ca. 27 \% (BRANDE 1980a) angegeben werden. Wie den Pollendiagrammen polnischer Arbeiten zu entnehmen ist, wurden auch in Zentralpolen sowie im Lednica Landschaftspark bei weitem nicht so hohe Haselwerte erreicht wie weiter im Westen entlang des unteren Odertals (vgl. MAKOHONIENKO 2001b).

Abgesehen von den insgesamt geringeren Haselwerten begann die Ausbreitung der Hasel am Großen Krebssee etwa 8.350 cal. B.C. und etwas weiter nördlich, am Felchowsee, ca. 8.050 cal. B.C. (JAHNS 2000). Im Nordosten Brandenburgs datierte ENDTMANN (1998) das Haselmaximum auf 7.650 cal. B.C., gleiches gilt für den Berliner Raum (BRANDE 1996). Unter Berücksichtigung der vorliegenden Untersuchungen und der in WOLTERS (2002) zusammengestellten Radiocarbondatierungen zur Einwanderung und Ausbreitung der Hasel zeichnet sich im Bereich des unteren Odertals eine von Südosten nach Nordwesten gerichtete Ausbreitung ab. Im südlichen unteren Wartatal, südöstlich vom Wustrowsee, 
erreichte die Hasel gegen $8.750 \mathrm{cal}$. B.C. ein erstes Maximum von ca. $100 \%$. Ein zweites Maximum $(80 \%)$ folgte um 6.950 cal. B.C.. Es fällt bereits in den Übergang zum Atlantikum.

Das frühe Erscheinen der Hasel im südlichen unteren Wartatal findet in den von RALSKAJASIEWICZOWA (1983) aufgestellten Isopollenkarten, denen zufolge die Hasel gegen 9.500 B.P. (ca. 11.000 cal. B.C.) bereits mit Werten bis zu $10 \%$ an der Gesamtpollensumme beteiligt war, seine Bestätigung.

WOLTERS (2002) fordert in diesem Zusammenhang weitere Untersuchungen zur Haselausbreitung an edaphisch vergleichbaren Standorten.

Die der Tabelle $11 \mathrm{zu}$ entnehmenden Angaben der vorliegenden Untersuchungen über die Dauer des Boreals, also über den Zeitraum zwischen Haselausbreitung bzw. Haseldominanz und Erlenausbreitung, weichen ganz erheblich voneinander ab. Die Angaben in der Literatur bezüglich der Dauer des Boreals zeigen ebenfalls erhebliche Abweichungen. So ermittelte Wolters (2002) für die Döbritzer Heide in Brandenburg eine Dauer von etwa 1.800 Jahren, bei BRANDE (1996) wurde die Dauer des Boreals für den Berliner Raum mit etwa 1.000 Jahren angegeben. JAHNS (2000) gibt für das östliche Brandenburg eine Dauer von 630 bzw. 830 Jahren an, und für die Niederlausitz nimmt man eine Dauer von 1.422 (Warven-)Jahren an (BITTMANN \& PASDA 1999).

$\mathrm{Zu}$ einem einheitlicheren Ergebnis gelangt man, wenn man, wie von WoLTERS (2002) vorgeschlagen, die Angaben über die Gesamtdauer von Präboreal und Boreal vergleicht. Hier schwanken die Angaben zwischen 2.500 Jahren (WOLTERS 2002), 2.342 WarvenJahren (BITTMANN \& PASDA 1999) und 2.300 Jahren (BRANDE 1996).

\subsection{Atlantikum}

Der Beginn des Älteren Atlantikums im Untersuchungsgebiet ist nach FIRBAS (1952) vor allem durch den Anstieg der Alnus-Kurve gekennzeichnet. Der Beginn dieses kontinuierlichen Anstiegs datiert am Wustrowsee mit etwa 7.660 cal. B.C. und stimmt gut mit der von JAHNS (2000) angegebenen Datierung von 7.460 cal. B.C. für den westlich angrenzenden Großen Krebssee überein. Im unteren Wartatal, in der Umgebung von Rybojady, ist der Anstieg der Alnus-Kurve nach Interpolation mit 7.000 cal. B.C. datiert. Möglicherweise ist dieses Alter zu jung, da die Ausgangsdaten (10810 BP und 8070 BP) nur auf geringen Mengen Kohlenstoff basieren und außerdem Abweichungen der $\delta^{13} \mathrm{C}$-Werte aufweisen (s. Tabelle 5). Der steile Anstieg der Erlen (von $4 \%$ auf $30 \%$ in knapp 120 Jahren) im 
Gebiet um Rybojady findet seine Erklärung einerseits in der geringen Sedimentationsrate von nur 0,07 cm pro Jahr, anderseits waren die Ausgangsbedingungen für Alnus hier durch die geringere Präsenz von Haselbeständen im Vergleich zum Wustrowsee deutlich besser. TOBOLSKI (1990) gibt den Beginn des Atlantikums im Lednica Landschaftspark mit 8.560 \pm 140 B.P. (ca. 7.540 cal. B.C.) an, am Gościąż-See datiert die Erlenausbreitung mit ca. 7.800 cal. B.C. (RALSKA-JASIEWICZOWA et al. 1998). Berücksichtigt man die mögliche Unsicherheit der Datierung von Rybojady, so bestätigen die übrigen Datierungen aller hier aufgeführten Lokalitäten die für Polen angenommene Erleneinwanderung von Nord- und Südosten in Richtung Westen siehe (RALSKA-JASIEWICZOWA 1983: Abb. 64, 65).

Der Übergang zum Jüngeren Atlantikum (VII) wird laut FIRBAS (1949) durch den Anstieg der Fraxinus-Werte gekennzeichnet und wurde im unteren Odertal, am Wustrowsee, mit etwa 5.760 cal. B.C. datiert. Damit stimmt die Datierung gut mit den von JAHNS (2000) am Großen Krebssee sowie am etwas nördlich gelegenen Felchowsee ermittelten Ergebnissen für den Beginn des Jüngeren Atlantikums, den sie mit 5.890 cal. B.C. angibt, überein. Nach KALIS \& MEURERS-BALKE (1998) lässt sich die Eschenausbreitung in Nordwesteuropa auf die Förderung durch die Bandkeramiker zurückführen, da die Eschen angeblich auf Schneitelung mit einer erhöhten Blütenproduktion reagieren. Im unteren Wartatal, wurde im Profil von Rybojady für den Fraxinus-Anstieg durch Interpolation ein Alter von $6.220 \mathrm{cal}$. B.C. errechnet. Danach erfolgte die Ausbreitung der Esche in Rybojady früher als in den nordwestlich gelegenen Gebieten. TOBOLSKI (1990) datiert die Ausbreitung der Eschen am Skrzetuszewski-See im Lednica Landschaftspark auf $7560 \pm 140$ B.P. (ca. 6.390 cal. B.C.). In Zentralpolen, am Gościąż-See breiteten sich die Eschen um 6.250 cal. B.C. aus (RALSKA-JASIEWICZOWA et al. 1998), ebenso am Swietokrzyskie-See, im Gebiet um Gniezno (MAKOHONIENKo 2001b). Im Pollendiagramm vom Pawski Ług ist eine Altersbestimmung nur mittels Extrapolation möglich. Das ermittelte Alter von 4.030 cal. B.C. erscheint im Vergleich mit den anderen Datierungen jedoch sehr jung und wird als unwahrscheinlich eingestuft.

\subsection{Subboreal}

Der Übergang vom Jüngeren Atlantikum zum Subboreal wird in vielen Fällen durch den „Ulmenfall“ gekennzeichnet. Als Ulmenfall wird der in den meisten Pollendiagrammen Nord- und Nordwesteuropas auffällige Rückgang der Ulmenwerte beschrieben. Nach WIETHOLD (1998) erfolgte der Rückgang der Ulmen im nördlichen und zentralen Mitteleuropa überwiegend synchron (um 4.000 cal. B.C. nach BEHRE \& KUČAN (1994)) 
und ist wahrscheinlich auf phytopathogene Ursachen zurückzuführen. Anthropogene, auf intensive Schneitelwirtschaft zurückgehende Ursachen schließt er aufgrund des zu der Zeit geringen Siedlungsdrucks mesolithischer Bevölkerungsgruppen aus. KUBITZ (2000) kommt in ihren Untersuchungen am Meerfelder Maar zu einem anderen Ergebnis. Sie nimmt an, dass der Ulmenfall (von $20 \%$ auf $4 \%$ in der Zeit von 4.350 bis 4.150 v.y. B.C.) aus der Kombination von klimatischen Veränderungen, durch menschliche Aktivitäten und nicht zuletzt natürlicher Waldentwicklung resultiert. In den vorliegenden Untersuchungen zeigt sich ebenfalls eine deutliche Bindung des Ulmenrückgangs an das vermehrte Auftreten von Siedlungszeigern, so dass eine durch den Menschen beeinflusste Förderung des Ulmenrückgangs nicht auszuschließen ist.

Die Tabelle 12 liefert eine Übersicht der Altersangaben des Ulmenfalles aus den hier untersuchten Lokalitäten sowie daran angrenzender Gebiete. Leider fehlen aus einigen Arbeiten die genauen Prozentangaben, so dass nicht immer ersichtlich ist, aus welchem Bereich des Rückgangs die Datierung stammt.

Der Übersicht ist zu entnehmen, dass der Rückgang der Ulmenwerte im unteren Wartatal, im westlichen unteren Odertal und im Gebiet um Berlin zwischen 3.940 und 3.780 cal. B.C. erfolgte. Eine Ausnahme stellt mit einem Alter von $4.350 \mathrm{cal}$. B.C. das Gebiet um den Wustrowsee dar. Bereits sehr früh setzte der Rückgang im Gebiet um Poznań am Lednica-See, in Zentralpolen am Gościąż-See und auf der Insel Wolin ein. Laut LATAŁOWA (1999) besteht aufgrund der früh einsetzenden und intensiven Besiedlung auf Wolin durchaus ein Zusammenhang zwischen dem vergleichsweise frühen Rückgang der Ulmenwerte und den Siedlungsaktivitäten.

Abgesehen von der Datierung des Ulmenfalles am Wustrowsee, die sehr gut mit der aus Nordwestdeutschland (KUBITZ 2000) übereinstimmt, zeigen alle anderen Datierungen erhebliche Abweichungen gegenüber den nordwestdeutschen, so dass die Ulmenkrankheit als alleinige Ursache in Frage gestellt werden muss und ein anthropogener Zusammenhang durchaus möglich ist.

Ein weiteres waldgeschichtliches Ereignis des Subboreals stellt die Einwanderung von Fagus und Carpinus dar. Die von der Höhe der Zählsumme abhängige empirische Pollengrenze von Fagus im östlichen unteren Odertal ist auf 4.340 cal. B.C. datiert. Entlang des unteren Wartatals wird diese Grenze erst ca. 700 bis 800 Jahre später ereicht. Carpinus ist im östlichen unteren Odertal seit 2.900 cal. B.C. anwesend. Im unteren Wartatal ist Carpinus bereits früher, nämlich seit ca. 3.400 cal. B.C. präsent. Auf die 
Ausbreitung von Fagus und Carpinus sowie auf den Vergleich mit benachbarten Untersuchungsgebieten wird in Kapitel 8 ausführlicher eingegangen.

\section{5 Älteres Subatlantikum}

Der Übergang vom Subboreal (VIII) zum Älteren Subatlantikum (IX) ist nach FIRBAS (1949) durch den Rückgang der Corylus- und Tilia-Werte charakterisiert. Den Datierungen zufolge setzte dieser Rückgang am Wustrowsee nach Interpolation ca. 1.060 cal. B.C. ein, am Krebssee erfolgte er knapp 200 Jahre später, gegen 870 cal. B.C.. Entlang des unteren Wartatals wurde der Rückgang der Hasel-Werte früher, nämlich mit ca. 1.170 cal. B.C. (Rybojady) bzw. 1.160 cal. B.C. (Pawski Ług) datiert. Da alle Altersangaben durch Interpolation berechnet wurden, sind die Angaben hinsichtlich ihrer Zuverlässigkeit kritisch zu bewerten. Lediglich die Datierung am Krebssee stimmt sehr gut mit den von JAHNS (2000) ermittelten Altersangaben für das westliche untere Odertal, ca. 830 cal. B.C. am Großen Krebssee und ca. 930 cal. B.C. am Felchowsee, überein. Der vergleichsweise frühe Rückgang der Hasel- und Lindenwerte südlich des unteren Wartatals wird auf die dort früher einsetzende Ausbreitung der Rotbuchen und Hainbuchen zurückgeführt. Besonders deutlich wird dies in den Pollendiagrammen aus dem Lednica-Landschaftspark, in denen die Ausbreitung der Hainbuchen mit einem deutlichen Rückgang der Hasel korreliert (vgl. MAKOHONIENKO 2001b; Tobolski 2001).

\subsection{Jüngeres Subatlantikum}

Der Beginn des Jüngeren Subatlantikums, der mit der mittelalterlichen Bevölkerungsexpansion zusammenfällt, wird durch ansteigende Anteile von Pinus und zunehmende Siedlungszeigern um ca. 1.050 cal. A.D. am Wustrowsee und ca. 1.270 cal. A.D. am Krebssee charakterisiert. Im unteren Wartatal wurde im Profil Rybojady der Beginn des Jüngeren Subatlantikums ebenfalls mit ca. 1.270 cal. A.D. datiert, im Profil Pawski Ług ergab sich mit ca. 1.130 cal. A.D. ein etwa 140 Jahre jüngeres Alter. Die ermittelten Datierungen passen sehr gut zu den siedlungsgeschichtlichen Befunden, nach denen laut HERRMANN (1989) und GRINGMUTH-DALLMER (1995) ab dem 12. Jahrhundert mit einem Landesausbau zu rechnen ist. 


\section{Zur Einwanderung und Ausbreitung von Fagus und Carpinus}

Rotbuche und die Hainbuche zählen zu den telokratischen Gehölzen, die sich im Zuge der natürlichen postglazialen Waldentwicklung erst in der zweiten Hälfte des Holozäns in ihren heutigen Verbreitungsarealen ausbreiteten. Sie mussten sich gegen die bereits bestehenden, ökologisch stabilen, mesokratischen Waldgesellschaften durchsetzen und benötigten u. a. daher sehr viel mehr Zeit von der Einwanderung bis zur Massenausbreitung als die proto- und mesokratischen Gehölze, die im späten Postglazial und frühen Holozän (fast) konkurrenzlos in un- bzw. kaum bewaldete Areale vorstoßen konnten (FIRBAS 1949; LANG 1994). Die Glazialrefugien der meisten mesokratischen und telokratischen Gehölze sind im Süden der Apenninen- und Balkan-Halbinsel zu suchen, so auch die Refugien der Hainbuche (FIRBAS 1949) und der Rotbuche (GRÜGER 1977). Um so erstaunlicher ist es, dass die Rotbuchen und Hainbuchen trotz der gleichen „Ausgangsposition“ vergleichsweise spät einwanderten. Ein bedeutender Aspekt, der bei der Ausbreitung der Gehölze zu berücksichtigen ist, ist neben der Lage der Glazialrefugien die Verbreitungsart (zoochor, anemochor, anthropochor) und die damit verknüpfte Verbreitungsgeschwindigkeit (HUNTLEY \& BIRKS 1983; LANG 1994). Die mittlere Verbreitungsgeschwindigkeit beträgt für die Hasel nach LANG (1994) etwa $1.500 \mathrm{~m}$ pro Jahr, für die Rotbuche nehmen HUNTLEY \& BIRKS (1983) nur $200 \mathrm{~m}$ bis $300 \mathrm{~m}$ pro Jahr an. Die Wandergeschwindigkeit der Hainbuche soll bis zum Subboreal etwa $100 \mathrm{~m}$ pro Jahr betragen haben, danach soll sie mit Zunahme des menschlichen Einflusses nach HUNTLEY \& BIRKS (1983) fast auf das zehnfache angestiegen sein.

Die Rotbuche verfügt nach ELLENBERG (1996) über eine sehr breite ökologische Amplitude, und sie vermag aufgrund ihrer ökologischen Ansprüche und ihrer Eigenschaft als Schattholzart viele andere Gehölzarten von deren Standorten zu verdrängen. In den meisten Gebieten Nord- und Mitteleuropas, in denen ein ozeanisch geprägtes Klima herrscht, gelangte die Rotbuche nach ihrer Einwanderung im Subboreal in den vorherrschenden Laubmischwäldern auf den nährstoffreichen, humosen und gut durchlüfteten und feuchten Böden schnell zur Dominanz und drängte die übrigen Laubholzarten wie Ulme, Linde und Eiche zurück (vgl. FIRBAS 1949; LANG 1994). Nur wenig später als die Rotbuche erfolgte die Einwanderung der Hainbuche. Sie erhebt fast die gleichen Ansprüche an ihr Habitat wie die Rotbuche, allerdings ist sie gegenüber Spätfrösten 
weniger empfindlich, so dass sie in den durch ein mehr kontinental geprägtes Klima gekennzeichneten Gebieten der Rotbuche überlegen ist (ELLENBERG 1996).

Im folgenden sollen die Einwanderung und Ausbreitung der Rotbuche und Hainbuche in den Untersuchungsgebieten östlich des unteren Odertals anhand der Profile des Wustrowsees und Krebssees und südlich des unteren Wartatals anhand der Profile vom Pawski Ług und von Rybojady näher betrachtet werden. Unter Berücksichtigung der in VOIGT (1996) erläuterten Problematik bezüglich der Feststellung, ab wieviel Prozent Baumpollenanteil die lokale Rotbuchen- bzw. Hainbuchenanwesenheit belegt ist, wurden die in LANG (1994) vorgeschlagenen Kriterien angewandt. Danach belegt das Erreichen der geschlossenen Pollenkurve die Einwanderung in das Untersuchungsgebiet und das Erreichen von Anteilen mehr als $1 \%$ der Baumpollensumme (hier mind. 500 PK) die regionale und lokale Ausbreitung der Art. Dabei muss berücksichtigt werden, dass die Pollenproduktion von Carpinus um das dreifache höher ist als diejenige von Fagus und eine Anwesenheit von Carpinus im Untersuchungsgebiet damit erst bei Anteilen von mehr als $1 \% \mathrm{zu}$ erwarten ist (vgl. ANDERSEN 1970; LANG 1994). Geht man jedoch davon aus, dass Carpinus sich im Unterwuchs der Eichenmischwälder ausbreitete, so ist bereits bei einer geschlossenen Pollenkurve von der Anwesenheit im Untersuchungsgebiet auszugehen.

\subsection{Fagus}

In der Tabelle 13 sind die Altersangaben der jeweiligen Fagus-Anteile für die verschiedenen Untersuchungsgebiete zur Übersicht zusammengestellt (vgl. Tabelle 14: CarpinusAnteile).

Der Nachweis von vereinzelten Rotbuchenpollenkörnern nahm östlich des unteren Odertals bereits ab ca. 6.270 cal. B.C. zu. Allerdings ist die Anwesenheit der Rotbuche im Untersuchungsgebiet damit noch nicht belegt. GROSSE-BRAUCKMANN (1978) konnte nachweisen, dass trotz Abwesenheit einer Art durchaus höhere Anteile im Pollenniederschlag erreicht werden können, z. B. durch Pollenverfrachtung.

Eindeutig belegt ist die Anwesenheit von Fagus am Wustrowsee durch das Erreichen der geschlossenen (empirischen) Pollenkurve ab 4.340 cal. B.C.. Damit erreichte sie das östliche untere Odertal früher als das untere Wartatal, wie der Beginn der geschlossenen Pollenkurve im Profil Rybojady gegen 3.580 cal. B.C. belegt. Am Pawski Ług (mit sehr hohen Pinus-Werten) schloss sich die Kurve ca. 100 Jahre später, um 3.470 cal. B.C.. 
Für das westliche untere Odertal nimmt JAHNS (2000) die Einwanderung von Fagus zwischen ca. 4.400 cal. B.C. und ca. 3.560 cal. B.C. an.

Obwohl die Rotbuche bereits früh das untere Odertal erreicht hatte, benötigte sie vergleichsweise lange, nämlich bis ca. 2.730 cal. B.C., um ihre Anteile an der Baumpollensumme auf mehr als $1 \%$ auszuweiten. Im unteren Wartatal erreichte sie im Profil Rybojady diese Anteile um 2.540 cal. B.C., d. h. sie breitete sich trotz der späteren Einwanderung schnell aus. Damit widersprechen einerseits die Ergebnisse dem von RALSKA-JASIEWICZOWA (1983) aufgezeigten Verlauf der Fagus-Ausbreitung im heutigen Polen, nach dem um 4.000 B.P. ( 2.500 cal. B.C.) das mittlere Odertal, das mittlere Wartatal sowie die zentralen Gebiete des Großpolnischen Tieflandes noch frei von Rotbuchen waren. Andererseits bestätigen die vorliegenden Untersuchungen die Annahme von RALSKA-JASIEWICZOWA (1983), nach der die Ausbreitung der Rotbuchen von Westen nach Osten erfolgte.

Das frühe Erreichen der 1\%-Grenze im Profil vom Pawski Ług (3.050 cal. B.C.) wurde hier nicht berücksichtigt, da die Altersangabe auf Interpolation von weit voneinander entfernt liegenden Datierungen beruht und daher mit einer größeren Ungenauigkeit behaftet ist.

Auffällig ist, dass die Ausbreitung der Rotbuche sowohl entlang des unteren Odertals als auch entlang des unteren Wartatals mit dem vermehrten Auftreten von Siedlungszeigern korreliert.

Am schnellsten breitete sich die Rotbuche entlang des unteren Oderlaufs, am Wustrowsee, aus. Hier benötigte sie etwa 2.300 Jahre, um ihren Anteil von $1 \%$ bis zur Eisenzeit auf mehr als $5 \%$ auszuweiten. Noch während der Eisenzeit verdoppelte sich ihr Anteil an der Baumpollensumme, so dass sie gegen 130 cal. B.C. bereits mehr als $10 \%$ erreichte. Allerdings sind bei derartig geringen Buchenpollenanteilen ausgedehnte Rotbuchenwälder, wie sie für Nordwest- und Mitteldeutschland typisch sind, nicht zu erwarten. Wahrscheinlich konnten sich lediglich auf den gut durchfeuchteten und durchlüfteten Standorten im Bereich der nährstoffreichen Grundmoränenböden kleinere Rotbuchenbestände entwickeln, während auf den trockeneren und nährstoffärmeren Standorten Eichenmischwälder vorherrschten. Erst während der Völkerwanderungszeit konnten die Buchen ihre Anteile auf Maximalwerte um 18,4 \% (bzw. 13,7 \% am Krebssee) ausweiten. Demnach dürften die Ursachen für die langsame und geringe Ausbreitung der Rotbuche 
nicht nur klimatisch, sondern auch anthropogen bedingt sein. Im Mittelalter setzte dann rodungsbedingt der Rückgang der Rotbuchen ein.

Entsprechend den Erwartungen erreichte die Rotbuche in den weiter südöstlich gelegenen Profilen von Rybojady und vom Pawski Ług einen noch geringeren Anteil an der Baumpollensumme als in den Profilen vom Wustrowsee und Krebssee. Die maximalen Anteile betragen in beiden Profilen nur ca. $5 \%$ der Baumpollensumme. Auch benötigte sie in diesem Gebiet mit ca. 3.100 Jahren noch mehr Zeit als im unteren Odertal, um ihren Anteil von $1 \%$ auf $5 \%$ zu erhöhen. Somit bestätigt sich die Annahme, dass die Rotbuche aufgrund der nach Osten zunehmenden Winterkälte und vor allem der zunehmenden Sommertrockenheit sowie durch die Zunahme von Spätfrösten im Frühjahr an Konkurrenzkraft einbüßte. Ihre größte Ausdehnung ereichte sie wie im unteren Odertal erst während der Völkerwanderungszeit.

\subsection{Carpinus}

Die Ausbreitung von Carpinus erfolgte nach GLIEMEROTH (1995) aus den Glazialrefugien über Osteuropa ins westliche Mitteleuropa. Den Nord- und Südosten Polens erreichte die Hainbuche nach den von RALSKA-JASIEWICZOWA (1983) entworfenen Isopollenkarten um ca. 7.000 B.P. ( ca. 5.850 cal. B.C.), anschließend breitete sie sich in Richtung Westen aus. Gegen 3.800 B.P. ( ca. 2.500 cal. B.C.) war Carpinus mit Ausnahme der Küstenregion sowie mit Ausnahme des unteren Oder- und Wartatals im gesamten (heutigen) Polen bereits mit Anteilen bis zu $2 \%$ vertreten, d.h. sie kam in den Gebieten vor (RalskaJASIEWICZOWA et al. 2003). Im unteren Odertal und im südlichen unteren Wartatal erreichte die Hainbuche diese Anteile erst ca. 500 Jahre später (RALSKA-JASIEWICZOWA 1983).

Entgegen der Erwartung trat Carpinus in den hier vorliegenden Pollendiagrammen nicht im am weitesten östlich gelegenen Untersuchungsgebiet (Rybojady) zum ersten Mal auf, sondern im Umkreis von Pawski Ług (Tabelle 14). Das Einsetzen der empirischen Pollenkurve datiert hier auf ca. 3.370 cal. B.C., während weiter östlich, im Profil von Rybojady, der Beginn der geschlossenen Carpinus-Kurve auf 2.700 cal. B.C. datiert. Sogar am Wustrowsee, wo der Kurvenschluss mit 2.900 cal. B.C. angegeben wird, trat Carpinus-Pollen früher auf als im Gebiet von Rybojady. Aufgrund der vergleichsweise hohen Pollenproduktion von Carpinus ist erst beim Erreichen von Anteilen mehr als $1 \%$ die Anwesenheit im Untersuchungsgebiet belegt. Dies gilt jedoch nur, wenn man 
annimmt, dass die Hainbuche die erste Baumschicht bildet. Aufgrund der geringen Hainbuchen-Anteile und vergleichsweise hohen Eichen-Anteile, ist davon auszugehen, dass Carpinus in den Eichenmischwäldern wahrscheinlich in der zweiten Baumschicht vorkam. Das bedeutet eine geringere Präsenz im Pollenniederschlag, so dass bereits bei einer geschlossenen Pollenkurve mit der Anwesenheit im Untersuchungsgebiet zu rechnen ist.

Am Wustrowsee setzte die Ausbreitung von Carpinus ca. 2.580 cal. B.C. ein. Mit einer Verzögerung von etwa 250 Jahren (ab 2.320 cal. B.C. ) trat sie im südlichen unteren Wartatal, in Rybojady, auf. Obwohl die Hainbuche im Pollendiagramm von Pawski Ług schon sehr früh eine geschlossene Pollenkurve erreichte, benötigte sie dort am längsten, um ihre Anteile auf $1 \%$ zu erhöhen (ca. 1.770 cal. B.C.).

Offensichtlich begünstigten am Wustrowsee die durch neolithische Siedler infolge von Rodungen und Waldweidenutzung hervorgerufenen Auflichtungen der Wälder die Etablierung der Hainbuchen, da ihre Einwanderung mit neolithischen Siedlungstätigkeiten korreliert (siehe W 5b). Die weitere Ausbreitung der Hainbuchen erfolgte am Wustrowsee jedoch sehr zögerlich. Es dauerte 2.000 Jahre, bis ihre Anteile im östlichen unteren Odertal von $1 \%$ auf $5 \%$ anstiegen, und weitere 1.000 Jahre, bis die Anteile während der Völkerwanderungszeit auf mehr als $10 \%$ anstiegen. Basierend auf der Annahme, dass mit zunehmender Kontinentalität die Konkurrenzkraft der Rotbuche nachlässt, stellt sich die Frage, warum sich die Hainbuche entlang des östlichen unteren Odertals nicht in viel stärkerem Maße ausbreitete, da ja die größte Konkurrentin, die Rotbuche, kaum verbreitet war? Möglicherweise beschränkten sich die für Rotbuche und Hainbuche günstigen Standorte auf relativ kleine, inselartige Flächen, so dass die geringe Ausbreitung beider Arten überwiegend auf edaphische Ursachen zurückzuführen ist.

Entlang des südlichen unteren Wartatals erfolgte die Einwanderung der Hainbuche zwar später als im unteren Odertal, sie breitete sich dort jedoch deutlich schneller aus. Dies belegen auch die Arbeiten aus dem Lednica Landschaftspark (TOBOLSKI 1990; МАКОНОNIENKO 2001b).

Im südlichen Wartatal erfolgte die Ausbreitung der Hainbuche in zwei Phasen, wie der Kurvenverlauf belegt. So erreichte sie im Umkreis von Rybojady bereits in der Bronzezeit, ca. 1.300 cal. B.C., ein erstes Maximum von mehr als $10 \%$. Bemerkenswert ist, dass dieses Maximum in einen Diagrammabschnitt fällt, der bronzezeitliche Siedlungsaktivitäten reflektiert (R 6c), so dass von einer anthropogenen Förderung der Hainbuchen in der 
Umgebung von Rybojady auszugehen ist. In der Umgebung von Pawski Ług erreichte die Hainbuche erst später, gegen Ende der Eisenzeit, ca. 90 cal. B.C., ein erstes Maximum, das, wenn man die Pinus-Anteile aus der Bezugssumme herausnimmt, fast $20 \%$ beträgt. Ebenfalls in die Eisenzeit datiert das erste Carpinus-Maximum am Wustrowsee. Beide Maxima fallen in eine Phase nachlassender Siedlungstätigkeiten.

Das zweite Hainbuchenmaximum datiert an allen Untersuchungspunkten in die Völkerwanderungszeit. Dies entspricht auch den Ergebnissen aus dem weiter östlich gelegenen Untersuchungsgebieten der großpolnischen-kujawischen Ebene, wie z. B. die Pollendiagramme aus dem Lednica Landschaftspark belegen (vgl. TOBOLSKI 1990; MAKOHONIENKO 1991; MiLECKA 1991). Entsprechend den Erwartungen setzte hier die Einwanderung der Hainbuche früher ein als im unteren Warta- und Odertal. Des weiteren übertreffen die Baumpollenanteile der Hainbuche bei weitem diejenigen der vorliegenden Untersuchungen. Am Skrzetuszeskie-See stiegen die Hainbuchenanteile in der Bronzezeit (1.890 cal. B.C.) auf ein erstes Maximum von mehr als $20 \%$ an. Der dann folgende Verlauf der Carpinus-Kurve reflektiert sehr eindrucksvoll Siedlungsphasen (Rückgang) und Regerationsphasen (Anstieg). Aufgrund ihres besonders guten Stockausschlagvermögens und ihrer häufigen Fruktifikation (SCHÜTT et al. 2002) konnte sich die Hainbuche in den Phasen nachlassender Besiedlung wahrscheinlich schnell regenerieren, wie die rasche Zunahme während der Völkerwanderungszeit belegt. Der gleiche Befund spiegelt sich in den Pollendiagrammen vom Lednica-See (MAKOHONIENKO 1991) und Głęboczek-See (MAKOHONIENKO 2001a).

Unter Berücksichtigung der vergleichsweise geringen Hainbuchen-Anteile in den vorliegenden Untersuchungen sind reine Hainbuchen-Wälder, wie sie TOBOLSKI (1990) u. a. im großpolnisch-kujawischem Gebiet, wie z. B. im Lednica-Landschaftspark, für die Bronzezeit annimmt, nicht zu erwarten. Entlang des südlichen unteren Wartatals war die Hainbuche wahrscheinlich nicht bestandsbildend, sondern bildete, wie auch für das untere Odertal angenommen wird, die zweite Baumschicht in den Eichen-Hainbuchenwäldern.

Die Rotbuchen- und Hainbuchen-Anteile der vorliegenden Pollendiagramme deuten an, dass offensichtlich nur wenige Kilometer südöstlich des unteren Odertals, im südlichen unteren Wartatal, die Rotbuche in ein Gebiet vorstieß, in dem die edaphischen Bedingungen außerhalb ihres ökologischen Optimums lagen. Durch die Zunahme der Kontinentalität gegenüber den weiter nordwestlich gelegenen Untersuchungsgebieten entlang des östlichen unteren Odertals (am Wustrowsee und Krebssee) büßte die Rotbuche noch mehr 
an Konkurrenzkraft ein, so dass reine Buchenwälder nur inselartig vorkamen, d. h. die Bedeutung der Rotbuche in den Waldgesellschaften ließ in Richtung Osten nach. Dies bestätigen auch RALSKA-JASIEWICZOWA et al. (2003), die in ihrer Arbeit u. a. die Ausbreitung von Fagus und Carpinus in Zusammenhang mit dem Rückgang von Corylus entlang eines Transektes von Nordwestdeutschland bis Nordostpolen aufzeigen. Sie verdeutlichen die zunehmende Bedeutungslosigkeit der Rotbuche in Richtung Osten und den gleichzeitig zunehmenden Einfluss von Carpinus in den oligokratischen Wäldern Nordostdeutschlands und Nordpolens. Allen der Arbeit zugrunde liegenden Lokalitäten ist ein Rückgang von Corylus während der Etablierung von Fagus und Carpinus gemein, wie er sich auch in den vorliegenden Pollendiagrammen widerspiegelt. Die Abbildung 6 zeigt den chronologischen Ablauf der Fagus- und Carpinus-Ausbreitung in Zusammenhang mit dem Corylus-Rückgang und der Zunahme menschlicher Aktivitäten. Nach RALSKAJASIEWICZOWA et al. (2003) erfolgte eine Differenzierung der mesokratischen Laubwaldgesellschaften zunächst aufgrund des zunehmenden menschlichen Einflusses neolithischer Siedler. Die anschließende Massenausbreitung von Fagus und Carpinus wurde dann laut MÜLLER (1969) und RALSKA-JASIEWICZOWA et al. (2003) durch die Zunahme der Humidität ab ca. 550 cal. B.C. ausgelöst und durch das Nachlassen der Siedlungsaktivitäten während der Völkerwanderungszeit begünstigt.

Nach LEUSCHNER (1999) bevorzugt die Rotbuche Standorte mit einer genügend mächtigen Humusauflage (A-Horizont) und einer ausreichenden Wasser- und Nährstoffversorgung. Es ist demnach vorstellbar, dass erst die Nutzung der Wälder durch neolithische und bronzezeitliche Bevölkerungsgruppen die notwendigen standörtlichen Voraussetzungen für die Etablierung der Rotbuche hervorbrachte. Dass das rezente Vorkommen von Buchenwäldern nur auf inselartige Standorte beschränkt ist, liegt einerseits daran, dass geeignete Buchen-Standorte seit der Intensivierung der Landwirtschaft und der fortschreitenden Erosion nährstoffreichen, humosen A-Horizonts infolge großflächiger Entwaldungen fehlen. LEUSCHNER (1999) nimmt an, dass Standorte mit nur einer gering entwickelten Humusauflage (A-Horizont) sowie sommerliche Trockenheit den Herrschaftsbereich der Rotbuche stark eingrenzen. Andererseits zeigen die vorliegenden Untersuchungen, dass die Rotbuche im östlichen unteren Odertal und im südlichen unteren Wartatal nie von so großer Bedeutung war, wie beispielsweise in Nordwestdeutschland. Aufgrund der nur geringen Baumpollenanteile ist davon auszugehen, dass entlang des östlichen Odertals Buchenwälder standörtlich bedingt nur inselartig vorkamen. Als Primärwälder sind hier 
linden- und ulmenreiche Eichenmischwälder anzusehen. MATUSZKIEWICZ (1984) nimmt als potentiell natürliche Vegetation jedoch artenreiche Buchenwälder (Melico-Fagetum) an.

Entlang des Wartatals traten Buchenbestände, wie schon früher von HeSMER (1933) vermutet, ebenfalls nur inselartig auf. Entsprechend den Bodenverhältnissen ist auf den extrem trockenen, nährstoffarmen Talsanden Kiefernwald als Primärwald anzusehen, dem auf etwas feuchteren und nährstoffreicheren Standorten Eichen beigemischt waren. Im Bereich der lehmigen, nährstoffreicheren Grundmoränenböden entwickelten sich artenreichere Eichenmischwälder, in denen vor allem Linden und Ulmen verbreitet waren. Hainbuchenwälder, wie TOBOLSKI (1990) sie für das großpolnisch-kujawische Gebiet annimmt, sind im südlichen unteren Wartatal bisher nicht nachweisbar. Wahrscheinlich breiteten sich die Hainbuchen nach der Auflichtung der Eichenmischwälder in der zweiten Baumschicht aus. Eine Förderung der Hainbuchen durch Niederwaldwirtschaft erfolgte seit der Eisenzeit (TOBOLSKI 1990). 


\section{Zusammenfassung}

Die vorliegende Arbeit liefert einen Beitrag zur holozänen Vegetationsentwicklung Nordwestpolens. Es wurden je zwei Standorte im östlichen unteren Odertal (Wustrowsee und Krebssee) und im südlichen unteren Wartatal (Pawski Ług und Rybojady) pollenanalytisch untersucht.

Die holozäne Vegetationsentwicklung entspricht an allen vier Standorten im wesentlichen der mitteleuropäischen Grundsukzession: Im unteren Odertal herrschten während des Präboreals lichte Birkenwälder vor, im südlichen unteren Wartatal dominierten BirkenKiefernwälder. Das Boreal war in beiden Untersuchungsgebieten durch eine hohe HaselPräsenz gekennzeichnet. Im Laufe des Atlantikums entwickelten sich auf den nährstoffreichen, feuchten lehmig-tonigen Standorten überwiegend linden- und ulmenreiche Eichenmischwälder. Auf den trockenen, sandigen Standorten waren vor allem Kiefernwälder verbreitet.

$\mathrm{Ab}$ dem Subboreal traten die standörtlichen Besonderheiten der Untersuchungsgebiete verstärkt hervor.

Im östlichen unteren Odertal breitete sich die Rotbuche nach ihrer Einwanderung um ca. 4.300 cal. B.C. nur sehr langsam aus. Am Wustrowsee erreichte sie in der Eisenzeit, am Krebssee erstmals nach der Völkerwanderungszeit Baumpollenanteile von mehr als $10 \%$.

Im südlichen unteren Wartatal wurde die Einwanderung der Rotbuche mit ca. 3.500 cal. B.C. datiert. Dort erreichte sie insgesamt nur einen Baumpollenanteil von $5 \%$. Die in den Untersuchungsgebieten vorliegenden edaphischen und klimatischen Bedingungen lagen offensichtlich außerhalb des ökologischen Optimums der Rotbuche, so dass die Rotbuche hier nie die Vorherrschaft erlangte.

Das östliche untere Odertal stellt demnach primär ein von linden- und ulmenreichen Eichenmischwäldern beherrschtes Gebiet dar. Während des Neolithikums griff der Mensch in die natürliche Waldsukzession ein, indem er durch Auflichtungen infolge von Waldweide und Rodung die Einwanderung und Ausbreitung der Rotbuche begünstigte. Ihr Vorkommen beschränkte sich jedoch, entsprechend den standörtlichen Besonderheiten der flachkuppigen Grundmoränenlandschaft, auf kleine, inselartige Bestände auf den nährstoffreicheren und gut durchlüfteten Standorten. Dies gilt ebenso für das südliche untere Wartatal. 
Entgegen der Erwartung, dass mit zunehmender Kontinentalität die Konkurrenzkraft der Rotbuche nachlässt und die Hainbuche zur dominierenden Baumart wird, entwickelten sich in beiden Untersuchungsgebieten trotz des geringen Konkurrenzdrucks keine ausgedehnten Hainbuchenwälder, wie sie weiter östlich aus der großpolnisch-kujawischen Ebene belegt sind. Statt dessen bildeten Hainbuchen wahrscheinlich die zweite Baumschicht in den durch anthropogene Nutzung stark degradierten Eichenmischwäldern der feuchten, nährstoffreichen Grundmoränenstandorte. Auf den trockenen, nährstoffarmen sandigen Standorten waren Kiefern verbreitet.

Die Ausbreitung der Hainbuche erfolgte im südlichen Wartatal in zwei Phasen und korreliert jeweils mit Phasen nachlassender Besiedlung. Ein erstes Maximum erreichte die Hainbuche bereits in der Bronzezeit, das zweite folgte, wie im unteren Odertal, in der Völkerwanderungszeit. 


\section{Literaturverzeichnis}

Aaby, B. (1986): Trees as anthropogenic indicators in regional pollen diagrams from eastern Denmark. In: K.-E. Behre (Hrsg.): Anthropogenic indicators in pollen diagrams. Rotterdam, Boston, Balkema: 73-93.

Ammann, B. (1984): Konzentrationen und Durchschnittsinflux der Pollenzonen im Spätglazial vom Lobsigensee.- Dissertationes Botanicae 72 (Festschrift Max Welten): 11-44.

Andersen, S. T. (1970): The relative pollen productivity and pollen representation of north european trees and correction factors for tree pollen spectra.- Danmarks Geologiske Undersøgelse 2(96): 1-99.

Behre, K.-E. (1978): Die Klimaschwankungen im europäischen Präboreal.- Petermanns Geographische Mitteilungen 122: 97-104.

Behre, K.-E. (1981): The interpretation of anthropogenic indicators in pollen diagrams.Pollen et Spores 23: 225-245.

Behre, K.-E. (1992): The history of the rye cultivation in Europe.- Vegetation History and Archaeobotany 1: 141-156.

Behre, K.-E. \& D. Kučan (1986): Die Reflektion archäologisch bekannter Siedlungen in Pollendiagrammen verschiedener Entfernungen - Beispiele aus der Siedlungskammer Flögeln, Nordwestdeutschland. In: K.-E. Behre (Hrsg.): Anthropogenic indicators in pollen diagrams. Rotterdam, Boston, Balkema: 95-114.

Behre, K.-E. \& D. Kučan (1994): Die Geschichte der Kulturlandschaft und des Ackerbaus in der Siedlungskammer Flögeln, Niedersachsen.- Probleme der Küstenforschung im südlichen Nordseegebiet 21: 1-227.

Benecke, N. \& D. Makowiecki (2002): Die Tierhaltung, Jagd, Fischfang und Sammelwirtschaft. In: E. Gringmuth-Dallmer \& L. Leciejewicz (Hrsg.): Mensch und Umwelt im Odergebiet. Mainz am Rhein, Philipp von Zabern: 337-343.

Beug, H.-J. (1957): Untersuchungen zur spätglazialen und frühpostglazialen Floren- und Vegetationsgeschichte einiger Mittelgebirge (Fichtelgebirge, Harz und Rhön).Flora 145: 167-211.

Beug, H.-J. (1961): Leitfaden der Pollenbestimmung - 1. Lieferung. Stuttgart, Fischer. 63.

Beug, H.-J. (1992): Vegetationsgeschichtliche Untersuchungen über die Besiedlung im Unteren Eichsfeld, Landkreis Göttingen, vom frühen Neolithikum bis zum Mittelalter.- Neue Ausgrabungen und Forschungen in Niedersachsen 20: 261-339.

Bittmann, F. \& C. Pasda (1999): Die Entwicklung einer Düne während der letzten 12000 Jahre. Untersuchungsergebnisse von Groß Lieskow (Stadt Cottbus) in der Niederlausitz.- Quartär 49/50: 39-54.

Björck, S., M. J. C. Walker, L. C: Cwynar, S. Johnson, K.-L. Knudsen, J. J. Lowe, B. Wohlfarth \& INTIMATE-members (1998): An event stratigraphy for the Last Termination in the Nort Atlantic region based on the Greenland ice-core record: a proposal by the INTIMATE-group.- Journal of Quaternary Science 13(4): 283292. 
Blytt, A. (1881): Die Theorie der wechselnden kontinentalen und insularen Kimate.Englers botanisches Jahrbuch 2: 1-50 u. 177-184.

Borówka, R., S. Fredrich, T. Heese, J. Jasanowska, R. Kochanowska, M Opęchowski, E. Stanecka \& W. Zyska (2004): Natur Westpommerns. Szczecin, Wołczkowo. Oficyna In Plus. 479.

Borówka, R. K., M. Latałowa, A. Osadczuk, J. Święta \& A. Witkowski (2001): Palaeogeography and Palaeoecology of Szczecin Lagoon. In: M. Latałowa (Hrsg.): 25th Bog Excursion North-West Poland, Part 1: Wolin Island and Drawa National Park (1-4th September 2001). Gdańsk, Department of Plant Ecology: 52-58.

Brande, A. (1980a): Die palynologische Erforschung des Quartärs in Berlin (West).- Acta Botanica Academiae Hungaricae 26: 55-60.

Brande, A. (1980b): Pollenanalytische Untersuchungen im Spätglazial und frühen Postglazial Berlins.- Verhandlungen des Botanischen Vereins der Provinz Brandenburg 115: 21-72.

Brande, A. (1988): Das Bollenfenn in Berlin (Tegel).- Telma 18: 95-135.

Brande, A. (1996): Type region D-s, Berlin. In: B. E. Berglund, H. J. B. Birks, M. RalskaJasiewiczowa \& H. E. Wright (Hrsg.): Palaeoecological events during the last 15.000 years. Chichester, John Wiley \& sons: 518-523.

Brande, A., P. Hoelzmann \& J. Klawitter (1990): Genese und Paläoökologie eines brandenburgischen Kesselmoores.- Telma 20: 27-54.

Brauer, A., T. Litt, J. F. W. Negendank \& B. Zolitschka (2001): Lateglacial varve chronology and biostratigraphy of lakes Holzmaar and Meerfelder Maar, Germany.- Boreas 30: 83-88.

Brose, F. (2002): Geologie und Entwicklung des Flusssystems. In: E. Gringmuth-Dallmer \& L. Leciejewicz (Hrsg.): Mensch und Umwelt im Odergebiet. Mainz am Rhein, Philipp von Zabern: 21-27.

Brose, F. \& K.-U. Heußner (2002): Zur Klimaentwicklung. In: E. Gringmuth-Dallmer \& L. Leciejewicz (Hrsg.): Mensch und Umwelt im Odergebiet. Mainz am Rhein, Philipp von Zabern: 27-33.

Brown, T. A., G. W. Farwell, P. M. Grootes \& F. H. Schmidt (1992): Radiocarbon AMS Dating of Pollen Extracted from Peat Samples.- Radiocarbon 34: 550-556.

Dansgaard, W., S. J. Johnsen, H. B. Clausen, S.O. Dahl, N. S. Gundestrup, C. U. Hammer, C. S. Hivdberg \& J. P. Steffensen (1993): Evidence for general instability of past climate from a 250-kyr ice-core record.- Nature 364: 218-220.

Domanski, G., A. Leube \& E. Schultze (2002): Die römische Kaiserzeit im Odergebiet. In: E. Gringmuth-Dallmer \& L. Leciejewicz (Hrsg.): Mensch und Umwelt im Odergebiet. Mainz am Rhein, Philipp von Zabern: 295-301.

Dörfler, W. (1989): Pollenanalytische Untersuchungen zur Vegetations- und Siedlungsgeschichte im Süden des Landkreises Cuxhaven; Niedersachsen.- Probleme der Küstenforschung im südlichen Nordseegebiet 17: 1-75.

Dörfler, W. (1990): Die Geschichte des Hanfanbaues in Mitteleuropa aufgrund palynologischer Untersuchungen und von Großrestnachweisen.- Prähistorische Zeitschrift 65(2): 218-244. 
Ehlers, J. (1994): Allgemeine und historische Quartärgeologie. Stuttgart, Enke. 358.

Ellenberg, H. (1996): Vegetation Mitteleuropas mit den Alpen. Stuttgart, Ulmer. 1096.

Endtmann, E. (1998): Untersuchungen zur spät- und nacheiszeitlichen Vegetationsentwicklung des Leckerpfuhls (Mönchsheider Sander, NE-Brandenburg).- Verhandlungen des Botanischen Vereins für Berlin und Brandenburg 131: 137-166.

Faegri, K., P. E. Kaland, \& K. Krzywinski (1989): Textbook of pollen analysis. Chichester, New York, John Wiley \& sons. 328.

Firbas, F. (1949): Waldgeschichte Mitteleuropas, Bd 1: Allgemeine Waldgeschichte. Jena, Fischer. 480.

Firbas, F. (1952): Waldgeschichte Mitteleuropas, Bd. 2: Waldgeschichte der einzelnen Landschaften. Jena, Fischer. 256.

Gediga, B. \& A. Mierzwinski (2002): Das östliche Odergebiet in der Bronze- und frühen Eisenzeit - Kulturen und Siedlungswesen. In: E. Gringmuth-Dallmer \& L. Leciejewicz (Hrsg.): Mensch und Umwelt im Odergebiet. Mainz am Rhein, Philipp von Zabern: 270-277.

Gliemeroth, A. K. (1995): Paläoökologische Untersuchungen der letzten 22000 Jahre in Europa - Vegetation, Biomasse und Einwanderungsgeschichte der wichtigsten Waldbäume.- Paläoklimaforschung 18: 252.

Goslar, T., M. Arnold, E. Bard, T. Kuc, M. F. Pazdur, M. Ralska-Jasiewiczowa, K. Rozanski, N. Tisnerat, A. Walanus, B. Wicik \& K. Wieckowski (1995): High concentration of atmospheric ${ }^{14} \mathrm{C}$ during the Younger Dryas cold episode.- Nature 377: 414-417.

Goslar, T., K. Balaga, K. Balaga, D. Arnold, N. Tisnerat, E. Starnawska, M. Kuzniarski, L. Chrost, A. Walanus \& K. Wieckowskie (1999): Climate-related variations in the composition of the Lateglacial and Early Holocene sediments of Lake Perespilno (eastern Poland).- Quaternary Science Reviews 18(7): 899-911.

Gramsch, B. (2002): Paläolithikum und Mesolithikum im Odergebiet - westlicher Teil. In: E. Gringmuth-Dallmer \& L. Leciejewicz (Hrsg.): Mensch und Umwelt im Odergebiet. Mainz am Rhein, Philipp von Zabern: 248-257.

Griesa, S. (1998): Siedlung. In: Facharbeitsgruppe "Bau- und Bodendenkmalpflege / Museumswesen Polens und Brandenburgs" (Hrsg.): Die Oder als Kulturlandschaft. Szczecin, ComGraph: 58-85.

Gringmuth-Dallmer, E. (1995): Siedlungshistorische Voraussetzungen, Verlauf und Ergebnisse des hochmittelalterlichen Landesausbaus im östlichen Deutschland. In: W. Rösener (Hrsg.): Grundherrschaft und bäuerliche Gesellschaft im Hochmittelalter. Göttingen, Vandenhoek \& Ruprecht: 320-358.

Grosse-Brauckmann, G. (1978): Absolute jährliche Pollenniederschlagsmengen an verschiedenen Beobachtungsorten in der Bundesrepublik Deutschland.- Flora 167: 209-247.

Grüger, E. (1977): Pollenanalytische Untersuchungen zur würmzeitlichen Vegetationsgeschichte von Kalabrien (Süditalien).- Flora 166: 475-489.

Haas, J. N. \& P. Rasmussen (1993): Zur Geschichte der Schneitel- und Laubfutterwirtschaft in der Schweiz - eine alte Landwirtschaftspraxis kurz vor dem Aussterben. 
In: C. Brombacher, S. Jacomet \& J. N. Haas (Hrsg.): Festschrift Zoller: Beiträge zu Philosophie und Geschichte der Naturwissenschaften, Evolution und Systematik, Íkologie und Morphologie, Geobotanik, Pollenanalyse und Archäobotanik. Berlin, Stuttgart, J. Cramer / Borntraeger: 469-489.

Harding, A. (1998): Reformation in Barbarian Europe, 1300-600 BC. In: B. Cunliffe (Hrsg.): Prehistoric Europe. Oxford, Oxford University Press: 304-335.

Henning, J. (1991): Germanen-Slawen-Deutsche. Neue Untersuchungen zum frühgeschichtlichen Siedlungswesen östlich der Elbe.- Prähistorische Zeitschrift 66: 119-133.

Herking, C. (1998): Vegetationsgeschichtliche Untersuchungen zur frühen Siedlungsgeschichte im Umkreis des Jues-Sees (Herzberg am Harz). Unveröffentlichte Diplomarbeit. Göttingen, Albrecht-von-Haller Institut für Pflanzenwissenschaften, Abteilung Palynologie und Quartärwissenschaften, Universität Göttingen. 75.

Herking, C. (2002): Der menschliche Einfluß auf die Vegetation im Umkreis des Ahlbecker Sees und des Kleinen Faulen Sees, Kreis Uecker-Randow, im Spiegel pollenanalytischer Untersuchungen.- Archäologische Berichte aus MecklenburgVorpommern 9: 16-25.

Herrmann, J. (1989): Landwirtschaft und Landesausbau in den slawischen Stammesgebieten. In: J. Herrmann (Hrsg.): Archäologie in der Deutschen Demokratischen Republik. Jena, Berlin, Urania-Verlag Leipzig: 249-256.

Hesmer, H. (1931): Untersuchungen zur Waldentwicklung in Pommern unter besonderer Berücksichtigung der Frage des natürlichen Fichtenvorkommens.- Forst- und Jagdwesen 10: 553-574.

Hesmer, H. (1932): Beitrag zur Waldentwicklungsgeschichte der Neumark.- Verhandlungen des Botanischen Vereins der Provinz Brandenburg 74: 349-354.

Hesmer, H. (1933): Die natürliche Bestockung und die Waldentwicklung auf verschiedenen märkischen Standorten.- Zeitschrift für Forst- und Jagdwesen 65: 505-651.

Heußner, K.-U. (2002): Siedlungsentwicklung und Holzbedarf. In: E. Gringmuth-Dallmer \& L. Leciejewicz (Hrsg.): Mensch und Umwelt im Odergebiet. Mainz am Rhein, Philipp von Zabern: 384-387.

Horoszko, S. (1998): Geschichte der Oderregion. In: Facharbeitsgruppe "Bau- und Bodendenkmalpflege / Museumswesen Polens und Brandenburgs" (Hrsg.): Die Oder als Kulturlandschaft. Szczecin, ComGraph: 12-26.

Hueck, K. (1929): Vegetationsstudien am Plötzendiebel bei Joachimsthal (Uckermark).Beiträge zur Denkmalpflege XIII: 1-231.

Hueck, K. (1931): Erläuterungen zur vegetationskundlichen Karte des Endmoränengebietes von Chorin (Uckermark). Mit Karte 1:25000.- Beiträge zur Naturdenkmalpflege in Berlin 14/2: 105-214.

Huntley, B. \& H. J. B. Birks (1983): An Atlas of Past and Present Pollen Maps for Europe: 0-13000 years ago. Cambridge, Cambridge University Press.667.

Huntley, B. \& I. C. Prentice (1996): July Temperatures in Europe from Pollen Data, 6000 Years Before Present.- Science 241: 687-690. 
Illig, H. \& E. Lange (1992): Vegetationsgeschichtliche und vegetationskundliche Untersuchungen im Rinnental bei Schuhlen-Wiese (Lausitz).- Verhandlungen des Botanischen Vereins für Berlin und Brandenburg 125: 5-18.

Iversen, J. (1944): Viscum, Hedera and Ilex as Climate Indicators. A constribution to the Post-Glacial Temperature Climate.- Danmarks Geologiske Undersøgelse 66: 463483.

Iversen, J. (1954): The late-glacial flora of Denmark and its relationship to climate and soil.- Danmarks Geologiske Undersøgelse, II.Raekke 80: 87-119.

Jahns, S. (2000): Late-glacial and Holocene woodland dynamics and land-use history of the Lower Oder valley, north-eastern Germany, based on two, AMS ${ }^{14} \mathrm{C}$-dated, pollen profiles.- Vegetation History and Archaeobotany 9(2): 111-123.

Jahns, S. \& C. Herking (2002): Zur holozänen und spätpleistozänen Vegetationsgeschichte westlich des unteren Oderlaufs. In: Mensch und Umwelt im Odergebiet. E. Gringmuth-Dallmer \& L. Leciejewicz (Hrsg.). Mainz, Verlag Philipp von Zabern: 33-49.

Jahns, S., C. Herking \& K. Kloss (2002): Landschaftsrekonstruktion entlang des westlichen unteren Oderlaufs anhand ausgewählter Pollenkurven aus acht Seeprofilen.- Greifswalder Geographische Arbeiten 26: 153-156.

Jessen, K. (1949): Studies in the Late Quaternary deposits and flora-history of Ireland.Proceedings of the Royal Irish Academy. 52B: 85-290.

Kalis, A. J. \& J. Meurers-Balke (1998): Die "Landnam"-Modelle von Iversen und TroelsSmith zur Neolithisierung des westlichen Ostseegebietes - ein Versuch ihrer Aktualisierung.- Prähistorische Zeitschrift 73(1): 1-24.

Kirk, H. (1960): Waldgeschichtliche Untersuchungen im Gebiet der Oberförsterei Siehdichum, Krs. Fürstenberg (Oder).- Wissenschaftliche Zeitschrift der Pädagogischen Hochschule Potsdam 6 (1/2): 159-170.

Kłosińska, E. (1996): Ziemie Polski północno-zachodniej w I i II okresie epolki brązu stan i potrzeby badań. In: L. Leciejewicz \& E. Gringmuth-Dallmer (Hrsg.): Człowiek a Środowisko w Środkowym i Dolnym Nadodrzu. Wrocław, Instytut Archeologii i Etnologii Polskiej Akademii Nauk: 67-80.

Knörzer, K.-H. (1978): Entwicklung und Ausbreitung des Leindotters (Camelina sativa s. 1.).- Berichte der Deutschen Botanischen Gesellschaft 91: 187-195.

Kobusiewicz, M. (2002): Die "Wojnowo-Region" - das Jäger-Sammler-Paradies. In: E. Gringmuth-Dallmer \& L. Leciejewicz (Hrsg.): Mensch und Umwelt im Odergebiet. Mainz am Rhein, Philipp von Zabern: 63-68.

Kobusiewicz, M., B. Nowaczyk \& I. Okuniewska-Novaczyk (1987): Late Vistulian Settlement in the Middle Odra Basin.- Prace Komisij Archeologicznej 5: 165182.

Komarek, J. \& B. Fott (1983): Das Phytoplankton des Süßwassers. Systematik und Biologie. 7. Teil, 1. Hälfte: Chlorophyceae, Ordnung: Chlorococcales.- Die Binnengewässer 16: 282-313.

Körber-Grohne, U. (1995): Nutzpflanzen in Deutschland. Hamburg, Nikol Verlagsgesellschaft mbH \& Co. KG.490. 
Kubitz, B. (2000): Die holozäne Vegetations- und Siedlungsgeschichte in der Westeifel am Beispiel eines hochauflösenden Pollendiagrammes aus dem Meerfelder Maar.- Dissertationes Botanicae 339: 106.

Kučan, D. (1986): Ältereisenzeitliche Kulturpflanzenreste aus Hamburg-Langenbek.Probleme der Küstenforschung im südlichen Nordseegebiet 16: 87-97.

Kulczycka-Leciejewiczowa, A. (2002): Neolithikum im Odergebiet. In: E. GringmuthDallmer \& L. Leciejewicz (Hrsg.): Mensch und Umwelt im Odergebiet. Mainz am Rhein, Philipp von Zabern: 257-263.

Lang, G. (1994): Quartäre Vegetationsgeschichte Europas. Methodik und Ergebnisse. Stuttgart, Gustav Fischer Verlag.462.

Lange, E. \& U. Liebetrau (1973): Die weichselglaziale und holozäne Talentwicklung im südlichen Jungmoränengebiet der DDR - morphogenetisch-stratigraphische Untersuchungen im Friedländer Tal bei Beeskow.- Wissenschaftliche Zeitschrift der Humboldt-Universität Berlin, Mathematisch-Naturwissenschaftliche Reihe 22: 671-676.

Latałowa, M. (1992): The Last 1500 Years on Wolin Island (NW Poland) in the Light of Paleobotanical Studies. In: J. P. Pals/J. Buurman/M. van der Veen (Hrsg): Festschrift for Professor van Zeist.- Review of Palaeobotany and Palynology 73: 213226.

Latałowa, M. (1999): Late Vistulian vegetation on Wolin Island (NW Poland) - The preliminairy results.- Quaternary Studies in Poland 16: 147-156.

Latałowa, M., (Hrsg.), (2001a): 25th Bog Excursion. North-west-Poland. Part I: Wolin Island and Drawa National Park (1.-4. September 2001). Gdańsk, Department of Plant Ecology, University of Gdańsk.

Latałowa, M. (2001b): Sicienko - History of vegetation. In: M. Latałowa (Hrsg.): 25th Bog Excursion, North-west Poland, Part I: Wolin Island and Drawa National Park (1-4th September 2001). Gdańsk, Department of Plant Ecology, Universitity of Gdańsk: 127-130.

Leciejewicz, L. \& E. Gringmuth-Dallmer (2002): Das frühe Mittelalter im Odergebiet. In: E. Gringmuth-Dallmer \& L. Leciejewicz (Hrsg.): Mensch und Umwelt im Odergebiet. Mainz am Rhein, Philipp von Zabern: 304-313.

Leszczycki, S., (Hrsg.), (1973-1978): Narodowy atlas Polski. Warszawa, Polska Akademii Nauk, Instytut Geografii.

Leube, A. (1991): Römische Kaiserzeit und Völkerwanderungszeit.- Führer zu archäologischen Denkmälern in Deutschland 23: 78-87.

Leube, A. (1996): Die eisenzeitliche Besiedlung des westlichen mittleren Odergebietes (6. Jahrh. v. Chr. - 6. Jahr. n. Chr.). In: L. Leciejewicz \& E. Gringmuth-Dallmer (Hrsg.): Człowiek a Środowisko w Środkowym i Dolnym Nadodrzu. Wrocław, Instytut Archeologii i Etnologii Polskiej Akademii Nauk: 127-142.

Leuschner, C. (1999): Einige kritische Anmerkungen zur Konstruktion der Potentiellen Natürlichen Vegetation.- Norddeutsche Naturschutz Akademie-Berichte 2/99: 8893. 
Lewczuk, J. (2002): Siedlungsdynamik in der vorrömischen Eisenzeit östlich der Oder. In: E. Gringmuth-Dallmer \& L. Leciejewicz (Hrsg.): Mensch und Umwelt im Odergebiet. Main am Rhein, Philipp von Zabern: 277-283.

Liedtke, H. (1981): Die nordischen Vereisungen in Mitteleuropa. Trier, Zentralausschuß für Deutsche Landeskunde. 307.

Linkowski, Z. (1998): Verkehr. In: Facharbeitsgruppe "Bau- und Bodendenkmalpflege / Museumswesen Polens und Brandenburgs" (Hrsg.): Die Oder als Kulturlandschaft. Szczecin, ComGraph: 154-173.

Litt, T. (1988): Untersuchungen zur spätglazialen Vegetationsentwicklung bei Dziekanowice (Umgebung Lednogora, Wielkopolska).- Acta Palaeobotanica 28 (1-2): 4960 .

Litt, T. (1994): Paläoökologie, Paläobotanik und Stratigraphie des Jungquartärs im nordmitteleuropäischen Tiefland. Berlin, Stuttgart, J. Cramer.185.

Litt, T. \& M. Stebich (1999): Bio- and chronostratigraphy of the lateglacial in the Eifel region, Germany.- Quaternary International 61: 5-16.

Lotter, A. F. (1989): Evidence of annual layering in Holocene sediments of Soppensee, Switzerland.- Aquatic Science 51(1): 19-30.

Lotter, A. F., B. Ammann \& M. Sturm (1992): Rates of change and chronological problems during the late- glacial period.- Climate Dynamics 6: 233-239.

Makohonienko, M. (1991): Materialy do postglacialnej historii roslinnosci okolic Lednogóry. Czesc II. Badania palinologiczne osadów Jeziora Lednickiego rdzen I/88 i Wal/87 [Beiträge zur postglazialen Vegetationsgeschichte im LednicaGebiet. Teil II. Palynologische Untersuchungen von Sedimenten des Lednicer Sees - Bohrkerne I/86 und Wal/87]. In: K. Tobolski (Hrsg.): Wstęp do paleoekologii Lednickiego Parku Krajobrazowego [Einführung in die Paläoökologie des Lednicer Landschaftsparks]. Poznań, Uniwersytetu im. Adama Mickiewicza: 6370 .

Makohonienko, M. (2001a): Gleboczek Lake - late Holocene history of vegetation in a forested area. In: K. Tobolski, M. Makohonienko, K. Milecka \& G. Kowalewski (Hrsg.): XXV. Moorexkursion North-Western Poland, 5-9th September 2001. Poznań, Adam Mickiewicz University Poznań: 85-89.

Makohonienko, M. (2001b): Gniezno - vegetation history and human impact in the sediments of Swietokrzyskie Lake. In: K. Tobolski, M. Makohonienko, K. Milecka \& G. Kowalewski (Hrsg.): XXV. Moorexkursion North-Western Poland, 5-9th September 2001. Poznań, Adam Mickiewicz University Poznań: 127-134.

Mangerud, J., S. T. Andersen, B. E. Berglund \& J. J. Donner (1974): Quaternary stratigraphy of Norden, a proposal for terminology and classification.- Boreas $\mathbf{3}$ : 109-128.

Marks, L. (2002): Last Glacial Maximum in Poland.- Quaternary Science Reviews 21: 103-110.

Matuszkiewicz, W. (1984): Die Karte der potentiell natürlichen Vegetation von Polen.Braun-Blanquetia 1: 99. 
Merkt, J. \& H. Müller (1999): Varve chronology and palynology of the Lateglacial in Northwest Germany from lacustrine sediments of Hämelsee in Lower Saxony.Quaternary International 61: 41-59.

Mierzwiński, A. (1996): Epoka brązu i początki epoki żelaza we wschodniej partii srodkowego Nadodrza (podstawy źródlowe i osadnicze, zarys dotychczasowych kierunków i perspektyw badawczych). In: L. Leciejewicz \& E. GringmuthDallmer (Hrsg.): Człowiek a Środowisko w Środkowym i dolnym Nadodrzu. Wrocław, Instytut Archeologii i Etnologii Polskiej Akademii Nauk: 109-126.

Milecka, K. (1991): Pollen analysis of lake sediments in Giecz - the state of the investigation. In: K. Tobolski (Hrsg.): Wstęp do paleoekologii Lednickiego Parku Krajobrazowego [Einführung in die Paläoökologie des Lednicer Landschaftsparks]. Poznań, Uniwersytetu im. Adama Mickiewicza: 147-150.

Milecka, K. (1998): Historia działalności człowieka w okolicaxch Giecza i Wagowa w świetle analizy pyłkowej.- Biblioteka Studiów Lednickich III: 43-95.

Milecka, K. (2001a): Anthropogenic changes in the vegetative cover of the past five thousand years in light of pollen analysis of lake sediments in Wielkopolska. In: B. Jackowiak \& W. Zuckowski (Hrsg.): Mechanisms of anthropogenic changes of the plant cover. Poznań, Adam Mickiewicz University: 55-68.

Milecka, K. (2001b): Pollen analysis of D1 core of sediments from Dury peatbog. In: K. Tobolski, M. Makohonienko, K. Milecka \& G. Kowalewski (Hrsg.): XXV Moorexkursion North-Western Poland, 5-9th September 2001. Poznań, Adam Mickiewicz University: 53-56.

Moore, P. D., J. A. Webb \& M. E. Collinson (1991): Pollen Analysis. Oxford, Blackwell Scientific Publications. 216.

Müller, H. M. (1967): Das Pollendiagramm "Serwest", ein Beitrag zur Wechselwirkung natürlicher und anthropogener Faktoren in der Vegetationsentwicklung.- Feddes Repertorium 74: 123-137.

Müller, H. M. (1969): Die spätpleistozäne und holozäne Vegetationsentwicklung im östlichen Tieflandsbereich der DDR zwischen Nördlichem und Südlichem Landrücken.- Wissenschaftliche Abhandlungen der Geographischen Gesellschaft der DDR 10: 155-165.

Müller, H. M. (1971): Untersuchungen zur Vegetationsentwicklung südlich von Berlin.Petermanns Geographische Mitteilungen 115: 37-45.

Neef, R. (2002): Ackerbau und Sammelwirtschaft. In: E. Gringmuth-Dallmer \& L. Leciejewicz (Hrsg.): Mensch und Umwelt im Odergebiet. Mainz am Rhein, Philipp von Zabern: 319-334.

Negendank, J. F. W., A. Brauer \& B. Zolitschka (1990): Die Eifelmaare als erdgeschichtliche Fallen und Quellen zur Rekonstruktion des Paläoenvironments.- Mainzer Geowissenschaftliche Mitteilungen 19: 235-262.

Nietsch, H. (1934): Waldgeschichtliche Untersuchungen im westlichen Ostpommern und in der angrenzenden Neumark.- Dohrniana 13: 1-137.

Noryskiewicz, B. (2001): Biskupin fortified settlement and its environment in the light of palynological studies. In: K. Tobolski, M. Makohonienko, K. Milecka \& G. 
Kowalewski (Hrsg.): XXV. Moorexkursion North-Western Poland 5-9th September 2001. Poznań, Adam Mickiewicz University Poznań: 77-84.

Oberdorfer, E. (1994): Pflanzensoziologische Exkursionsflora. Stuttgart, Ulmer. 1050.

Okuniewska-Novaczyk, I. (1992): Roslinnosc Poznoglacjalna Stanowiska Zarsko Kolo Cedyni. In: T. Szczypek (Hrsg.): Wybrane Zagadniemia Geomorfologii Eolicznes. 106-113.

Peglar, S. M. \& H. J. B. Birks (1993): The mid-Holocene Ulmus fall at Diss Mere, SouthEast England - disease and human impact?- Vegetation History and Archaeobotany 2: 61-68.

Peglar, S. M., S. C. Fritz, T. Alapieti, M. Saarnisto \& H. J. B. Birks (1984): Composition and formation of laminated sediments in Diss Mere, Norfolk, England.- Boreas 13: $13-28$.

Perry, I. \& P. D. Moore (1987): Dutch elm disease as an analogue of Neolithic elm decline.- Nature 326 (Nr. 6108): 72-73.

Pott, R. (1986): Der pollenanalytische Nachweis extensiver Waldbewirtschaftungen in den Haubergen des Siegerlandes. In: K.-E. Behre (Hrsg.): Anthropogenic indicators in pollen diagrams. Rotterdam, Boston, A.A.Balkema: 125-134.

Pott, R. (1990): Die Haubergswirtschaft im Siegerland - Vegetationsgeschichte, extensive Holz- und Landnutzungen im Niederwaldgebiet des südwestfälischen Berglandes. In: Wilhelm-Münker-Stiftung (Hrsg.): Die Haubergswirtschaft im Siegerland. Siegen: 6-40.

Pott, R. (2000): Palaeoclimate and vegetation - long-term vegetation dynamics in central Europe with particular reference to beech.- Phytocoenologia 30: 285-333.

Punt, W. (1976): The Northwest European pollen flora 1. Amsterdam, Elsevier. 145.

Punt, W. \& S. Blackmore (1991): The Northwest European pollen flora 6. Amsterdam, Elsevier. 275.

Punt, W., S. Blackmore, \& G. C. S. Clarke (1991): The Northwest European pollen flora 5. Amsterdam, Elsevier. 154.

Punt, W., S. Blackmore \& P. P. Hoen (1995): The northwest European pollen Flora. Amsterdam, Elsevier.

Punt, W. \& G. C. S. Clarke (1980): The Northwest European pollen flora 2. Amsterdam, Elsevier. 265.

Punt, W. \& G. C. S. Clarke (1981). The Northwest European pollen flora 3. Amsterdam, Elsevier.138.

Punt, W. \& G. C. S. Clarke (1984): The Northwest European pollen flora 4. Amsterdam, Elsevier. 369.

Ralska-Jasiewiczowa, M. (1983): Isopollen maps for Poland: 0-11000 years B.P.- New Phytologist 94: 133-175.

Ralska-Jasiewiczowa, M., T. Goslar, T. Madeyska, \& L. Starkel (Hrsg.) (1998): Lake Gościąż, Central Poland. A monographic study. Kraków, W. Szafer Institute Botany, Polish Academy of Sciences. 340. 
Ralska-Jasiewiczowa, M., D. Nalepka \& T. Goslar (2003): Some problems of forest transformation at the transition to the oligocratic/Homo sapiens phase of the Holocene interglacial in northern lowlands of central Europe.- Vegetation History and Archaeobotany 12: 233-247.

Ralska-Jasiewiczowa, M. \& B. van Geel (1992): Early human disturbance of the natural environment recorded in annually laminated sediments of lake Gościąż, central Poland.- Vegetation History and Archaeobotany 1: 33-42.

Ralska-Jasiewiczowa, M., B. van Geel \& D. Demske (1998): Holocene regional vegetation history recorded in the Lake Gościąż sediments. In: M. Ralska-Jasiewiczowa, T. Goslar, T. Madeyska \& L. Starkel (Hrsg.): Lake Gościąż, Central Poland. Kraków, W. Szafer Institute Botany, Polish Academy of Sciences: 202-219.

Ralska-Jasiewiczowa, M., B. van Geel, T. Goslar \& D. Demske (1992): The record of the Late Glacial/Holocene transition in the varved sediments of Lake Gościąż, central Poland.- Sveriges Geologiska Undersökning 81: 257-268.

Rüb, R. (1998): Wirtschaft und Arbeit. In: Facharbeitsgruppe "Bau- und Bodendenkmalpflege/Museumswesen Polens und Brandendurgs" (Hrsg.): Die Oder als Kulturlandschaft. Szczecin, ComGraph: 106-154.

Scamoni, A. (1988): Gedanken über die Verbreitung der Kiefer im Tiefland.- Forstarchiv 59: $173-180$.

Schleicher, J. (2002). Einwanderung und Ausbreitung von Rotbuche und Hainbuche in Deutschland - Datierung und Deutung pollenanalytischer Befunde aus dem Postglazial. Unveröffentlichte Staatsexamenarbeit. Göttingen, Albrecht-von-Haller Institut für Pfanzenwissenschaften, Abteilung Palynologie und Quartärwissenschaften, Universität Göttingen: 126.

Schrage, G. E. (1995): Ur- und Frühgeschichte. In: I. Materna \& W. Ribbe (Hrsg.): Brandenburgische Geschichte. Berlin, Akademie-Verlag: 45-84.

Schultze, E. (1998): Zum mittleren und unteren Odergebiet als Natur- und Wirtschaftsraum während der römischen Kaiserzeit. Berichte zum Oder-Projekt. RömischGermanische Kommission des Deutschen Archäologischen Institutes zu Frankfurt am Main (Hrsg.). Berlin: 139-151.

Schütt, P., H. J. Schuck \& B. Stimm (Hrsg.) (2002): Lexikon der Baum- und Straucharten. Hamburg, Nikol Verlagsgesellschaft mbH \& Co. KG. 581.

Sernander, R. (1908): On the evidence of Postglacial changes of climate furnished by the peat-mosses of Northern Europe.- Geologiska Föreningens i Stockholm förhandlingar 30: 465-473.

Sherratt, A. (1998): The Emergence of Elites: Earlier Bronze Age Europe, 2500-1300 BC. In: B. Cunliffe (Hrsg.): Prehistoric Europe. Oxford, New York, University Press: 244-276.

Smettan, H. (1989): Der Cannabis/Humulus-Pollentyp und seine Auswertung im Pollendiagramm. In: U. Körber-Grohne \& H. Küster (Hrsg.): Archäobotanik. Symposium der Universität Hohenheim (Stuttgart) vom 11.-16. Juli 1988. Berlin, Stuttgart, J. Cramer: 25-40. 
Stebich, M. (1999): Palynologische Untersuchungen zur Vegetationsgeschichte des Weichsel-Spätglazial und Frühholozän an jährlich geschichteten Sedimenten des Meerfelder Maares (Eifel).- Dissertationes Botanicae 320: 127.

Stockmarr, J. (1971): Tablets with spores used in absolute pollen analysis.- Pollen et Spores 13: 615-621.

Stuiver, M., P. J. Reimer \& E. Bard (1998): INTCAL 98 radiocarbon age calibration, 24,000 - 0 cal. BP.- Radiocarbon 40(3): 1041-1083.

Succow, M. (2001): Kennzeichnung und Typisierung. In: H. Joosten \& M. Succow (Hrsg.): Landschaftsökologische Moorkunde. Stuttgart, Schweizerbart: 229-252.

Tobolski, K. (1990): Paläoökologische Untersuchungen des Siedlungsgebietes im Lednica Landschaftspark (Nordwestpolen).- Offa 47: 109-131.

Tobolski, K. (Hrsg.) (1991): Wstęp do paleoekologii Lednickiego Parku Krajobrazowego. Poznań, Wydawnictwo Naukowe Uniwersytetu im. Adama Mickiewicza.

Tobolski, K. (2001): Lake Skrzetuszewskie. In: K. Tobolski, M. Makohonienko, K. Milecka \& G. Kowalewski (Hrsg.): XXV. Moorexkursion North-Western Poland, 5-9th September 2001. Poznań, Adam Mickiewicz University Poznań: 100-104.

Tobolski, K. \& T. Litt (1994): Vorallerödzeitliche Seeablagerungen in Wielkopolska (Großpolen).- Dissertationes Botanicae 224: 487-496.

Tobolski, K. \& I. Okuniewska-Novaczyk (1989): Type Region P-r: Poznań-GnieznoKujawy Lake District.- Acta Palaeobotanica 29(2): 77-89.

Troels-Smith, J. (1960): Ivy, Mistletoe and Elm, Climate Indicators - Fodder Plants.Danmarks Geologiske Undersøgelse 4(4): 1-32.

van Geel, B. (1978): A paleoecological study of Holocene peat bog sections in Germany and the Netherlands.- Review of Palaeobotany and Palynology 25: 1-120.

van Geel, B. \& H. Renssen (1998): Abrupt Climate Change around 2,650 BP in NorthWest Europe: Evidence for Climatic Teleconnections and a tentative Explanation. In: A. S. Issar \& N. Brown (Hrsg.): Water, Environment and Society in Times of Climatic Change. Amsterdam, Kluwer Academic Publishers: 21-41.

Voigt, R. (1996): Paläolimnologische und vegetationsgeschichtliche Untersuchungen an Sedimenten aus Fuschlsee und Chiemsee (Salzburg und Bayern).- Dissertationes Botanicae 270: 1-303.

Voß, H. J. (1984): Germanisch-Slawische Kontakte im mittleren Odergebiet. Interaktionen der mitteleuropäischen Slawen und anderer Ethnika im 6.-10. Jh.- Berlin, Nitra: 285-290.

Wagenitz, G. (1955): Pollenmorphologie und Systematik in der Gattung Centaurea L. s.l.Flora 142: 213-346.

Walter, H. \& H. Lieth (1960-1967): Klimadiagramm-Weltatlas. Jena, G. Fischer.

Wechler, K.-P. (1996): Zur Besiedlungsgeschichte des Odergebietes vom Spätpaläolithikum bis zum frühen Neolithikum. In: L. Leciejewicz \& E. Gringmuth-Dallmer (Hrsg.): Człowiek a Środowisko w Środkowym i Dolnym Nadodrzu. Wrocław, Instytut Archeologii i Etnologii Polskiej Akademii Nauk: 27-40. 
Wesołowski, S. (1996): Stan badań nad osadnictwem kultury łużyckiej Pomorza zachodniego. In: L. Leciejewicz \& E. Gringmuth-Dallmer (Hrsg.): Człowiek a Środowisko w Środkowym i Dolnym Nadodrzu. Wrocław, Instytut Archeologii i Etnologii Polskiej Akademii Nauk: 81-96.

Wiethold, J. (1998): Studien zur jüngeren postglazialen Vegetations- und Siedlungsgeschichte im östlichen Schleswig-Holstein.- Universitätsforschungen zur prähistorischen Archäologie 45: 365.

Willerding, U. (1986a): Aussagen zur Pollenanalyse und Makrorestanalyse zu Fragen der frühen Landnutzung. In: K.-E. Behre (Hrsg.): Anthropogenic indicators in pollen diagrams. Rotterdam, Boston, A.A.Balkema: 135-151.

Willerding, U. (1986b): Zur Geschichte der Unkräuter Mitteleuropas. Neumünster, Wachholtz Verlag. 382.

Willerding, U. (2003): Grundlagen der landwirtschaftlichen Produktion.- Beiträge zur Urund Frühgeschichte Mitteleuropas 14: 3-33.

Winnige, B. \& W. Rohlfs (1994): Karte und Beschreibung der Exkursionsroute.- Zentrum für Agrarlandschafts- und Landnutzungsforschung-Bericht 14: 147-151.

Woldstedt, P. (1974): Norddeutschland und angrenzende Gebiete im Eiszeitalter. Stuttgart, Koehler. 500.

Wolters, S. (1999): Spät- und postglaziale Vegetationsentwicklung im Bereich der Fercher Berge südwestlich von Potsdam.- Gleditschia 27: 25-44.

Wolters, S. (2002): Vegetationsgeschichtliche Untersuchungen zur spätglazialen und holozänen Landschaftsentwicklung in der Döbritzer Heide (Brandenburg).- Dissertationes Botanicae 366: 157.

Zolitschka, B. \& J. F. W. Negendank (1993): Lago Grande di Monticchio (southern Italy) - a high resolution sedimentary record of the last 70.000 years. In: J. F. W. Negendank \& B. Zolitschka (Hrsg.): Paleolimnology of European Maar Lakes, Lecture Notes in Earth Sciences: 277-288. 


\section{Anhang}

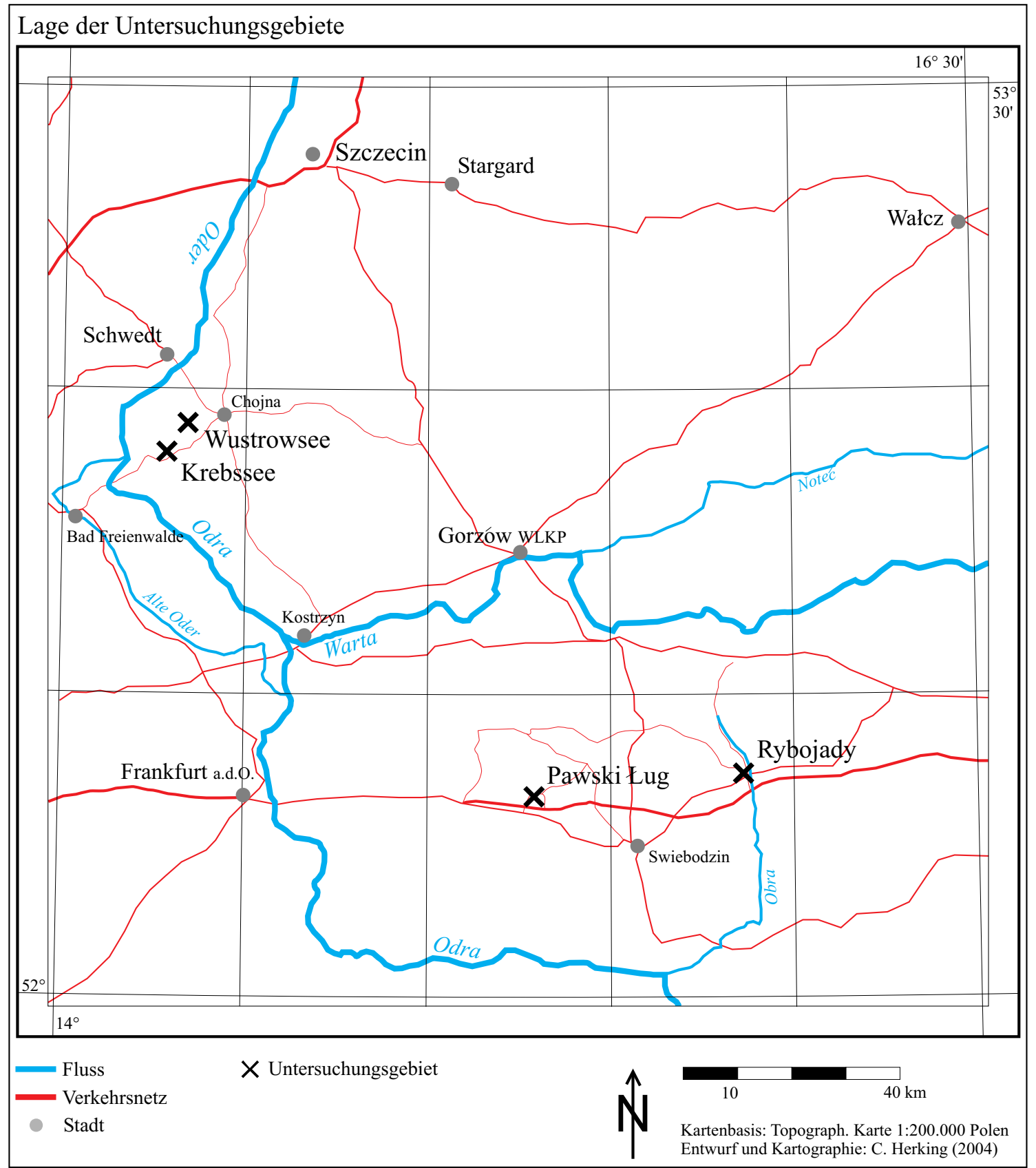

Karte 1: Lage der Untersuchungsgebiete (vgl. Karte 3-5) 


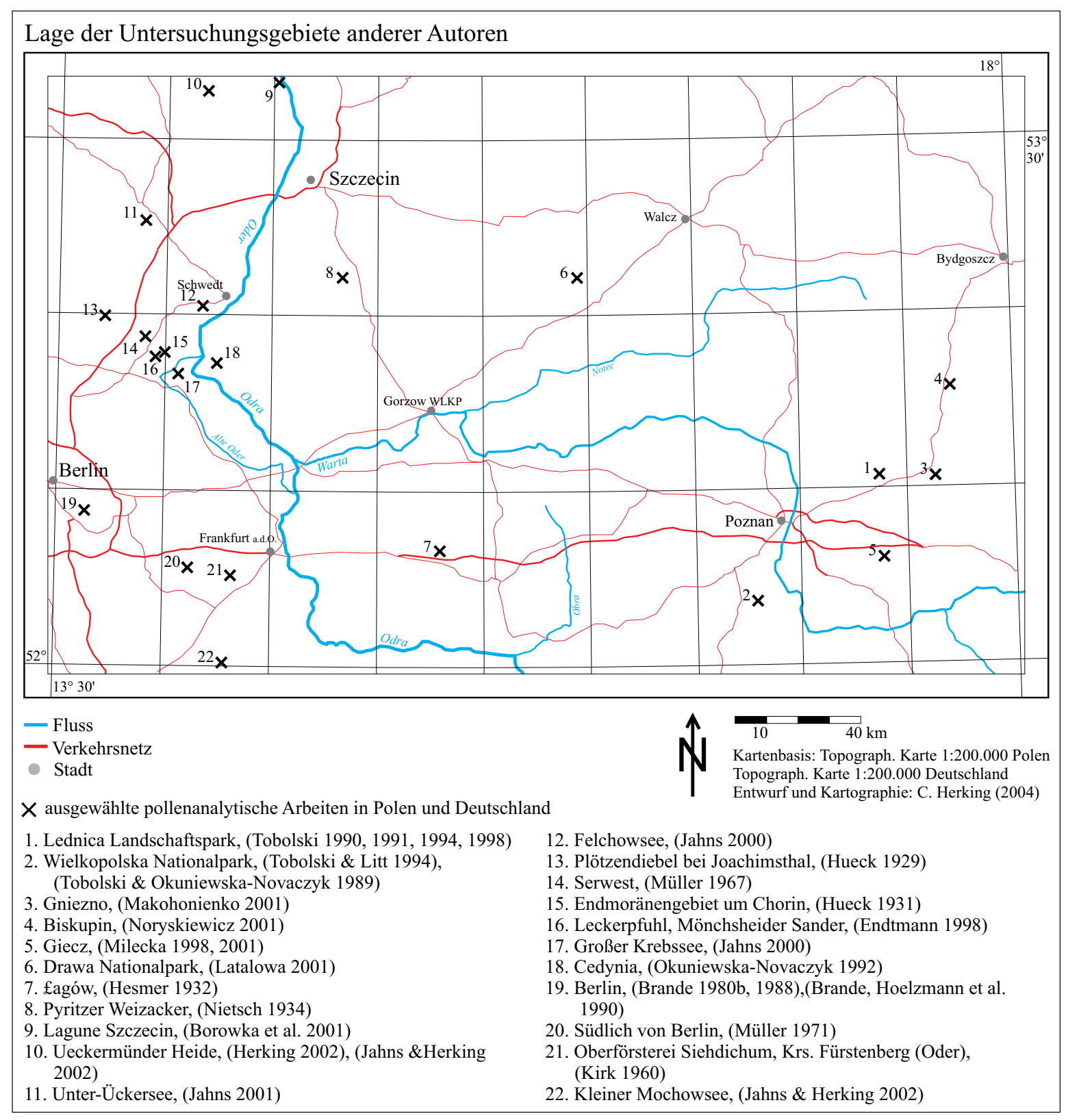

Karte 2: Übersichtskarte zur Lage ausgewählter pollenanalytischer Arbeiten in Polen und Deutschland 


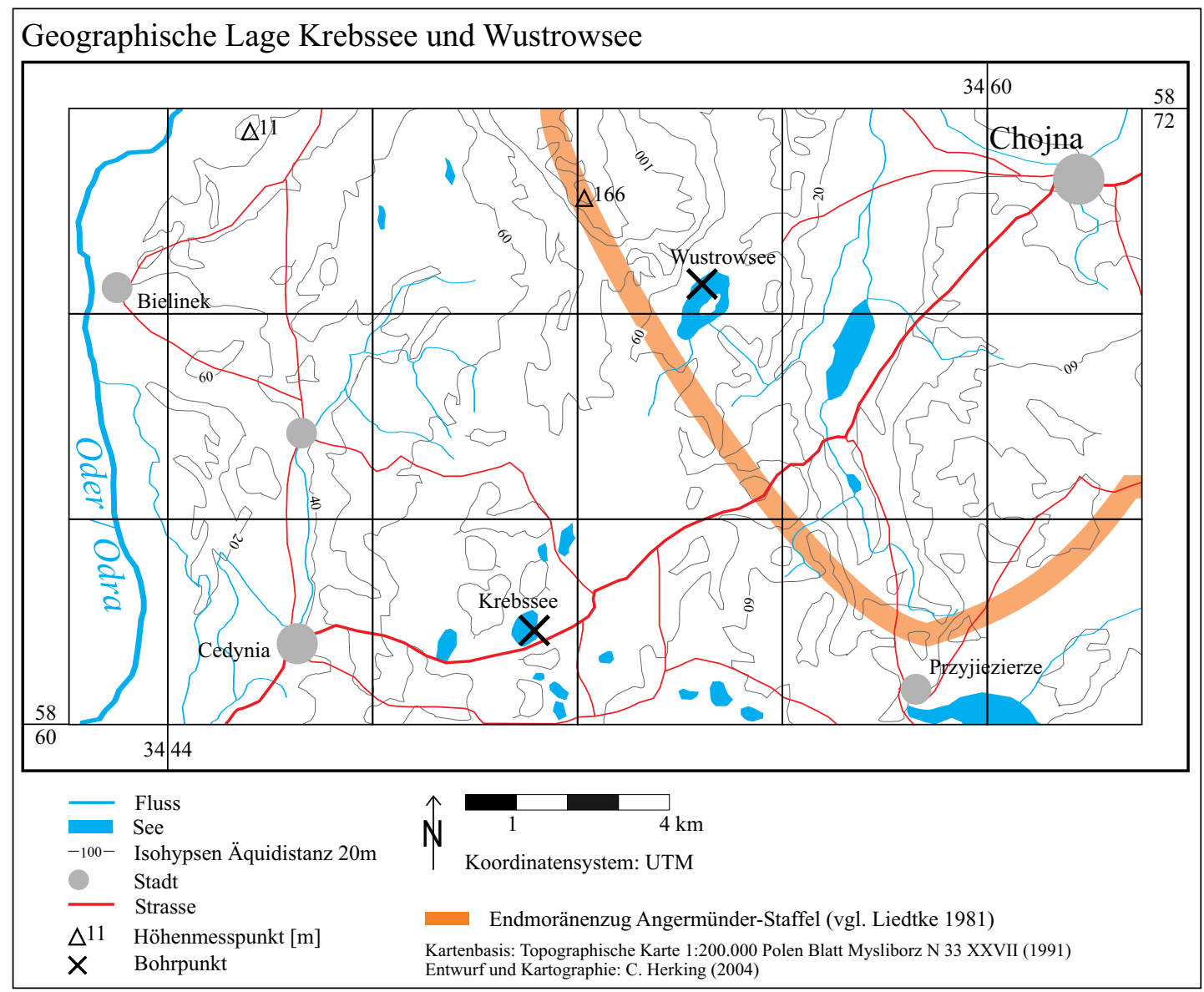

Karte 3: Die geographische Lage vom Krebssee und Wustrowsee

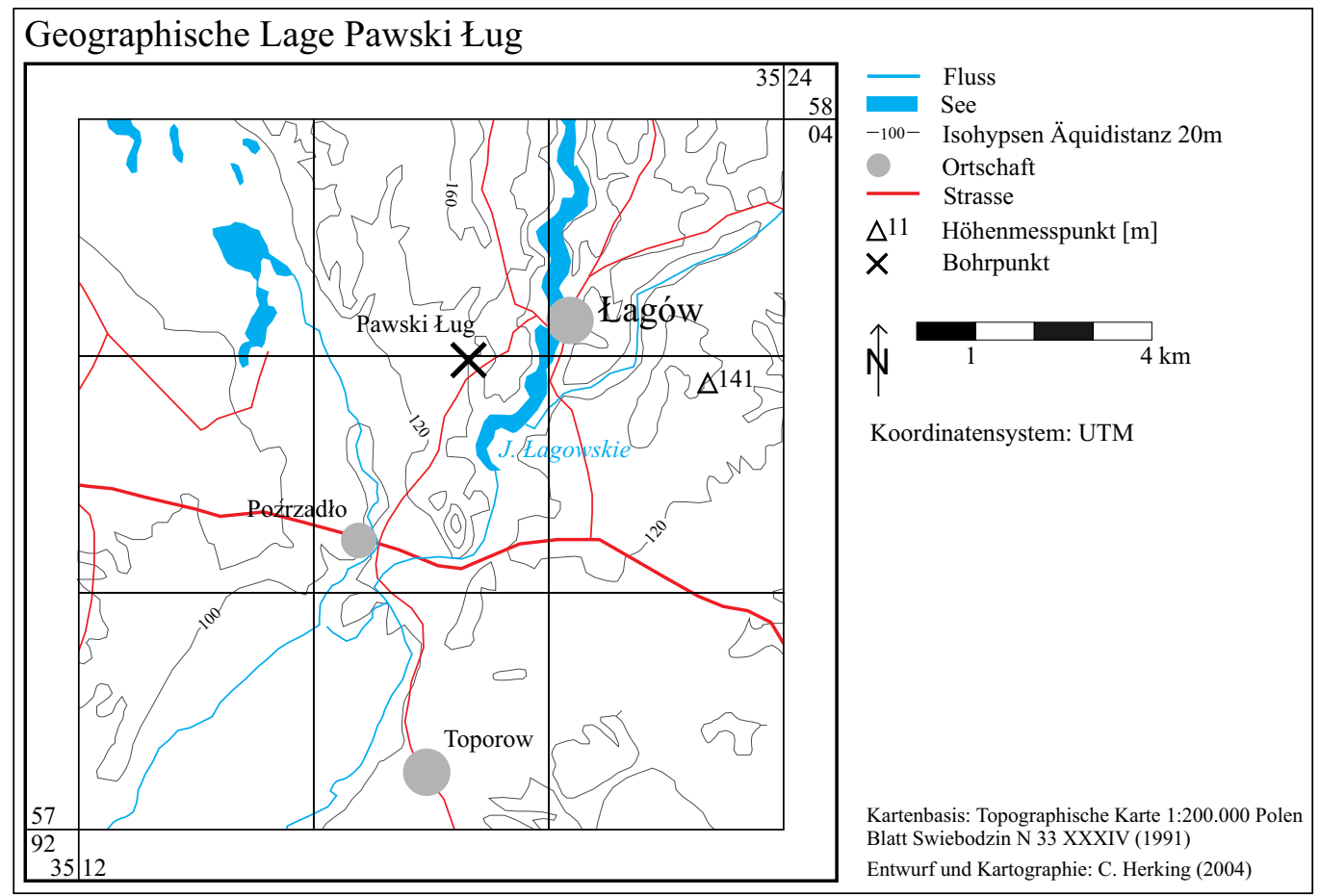

Karte 4: Die geographische Lage von Pawski Ług 


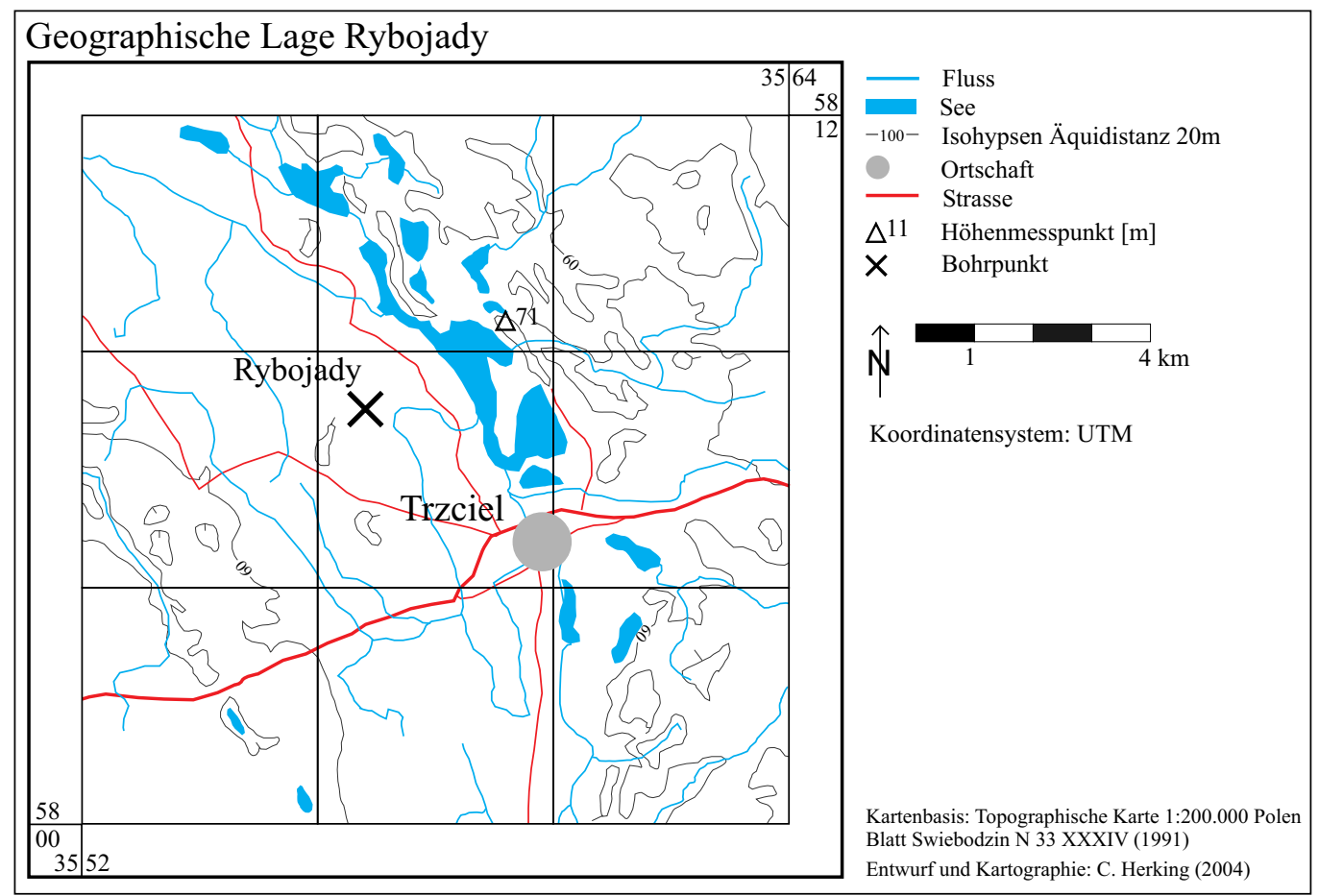

Karte 5: Die geographische Lage von Rybojady 


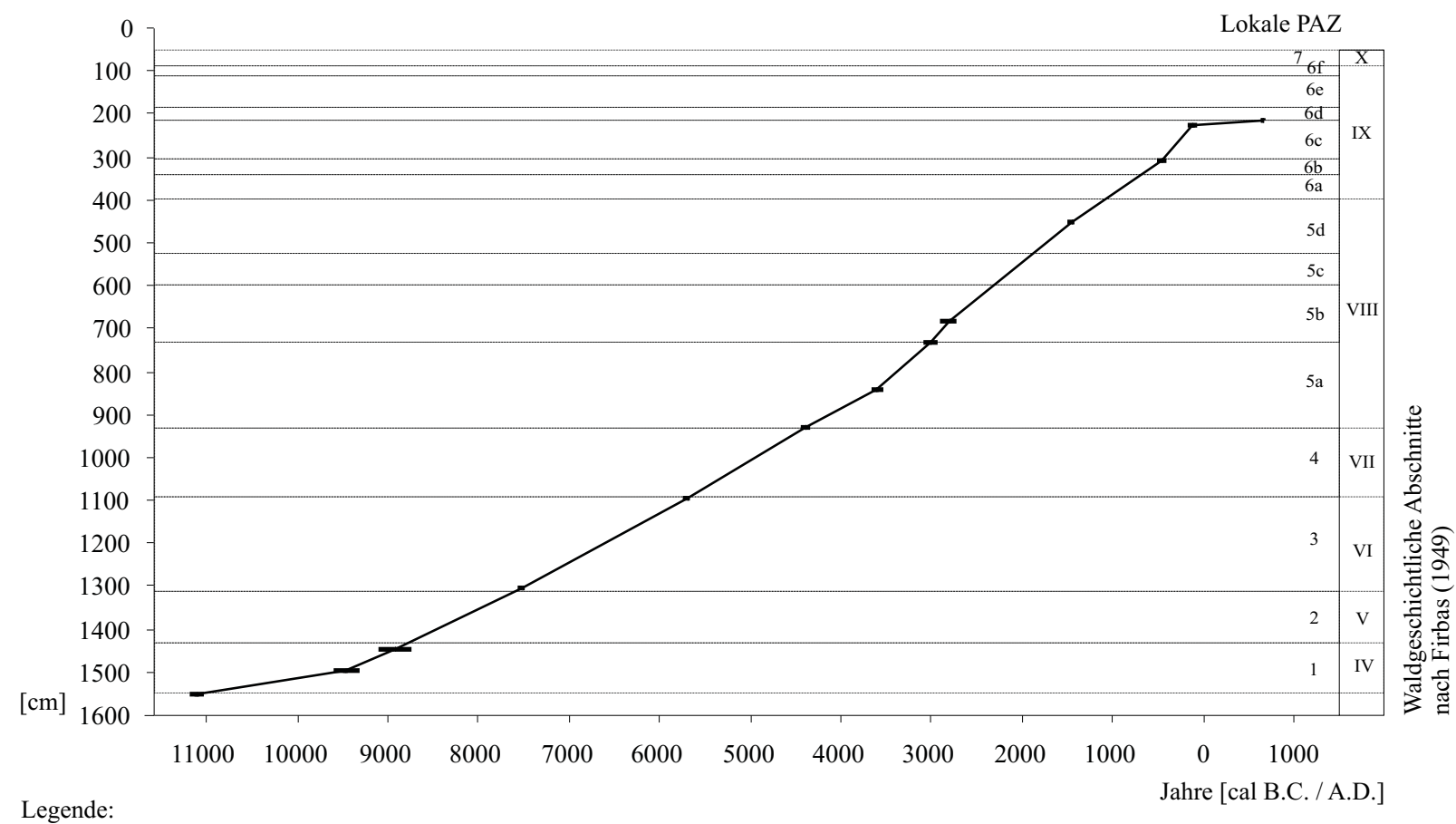

Legende:

— Sedimentzuwachskurve nach linearer Interpolation

Abbildung 1: Zeit-Tiefen-Diagramm vom Profil Wustrowsee

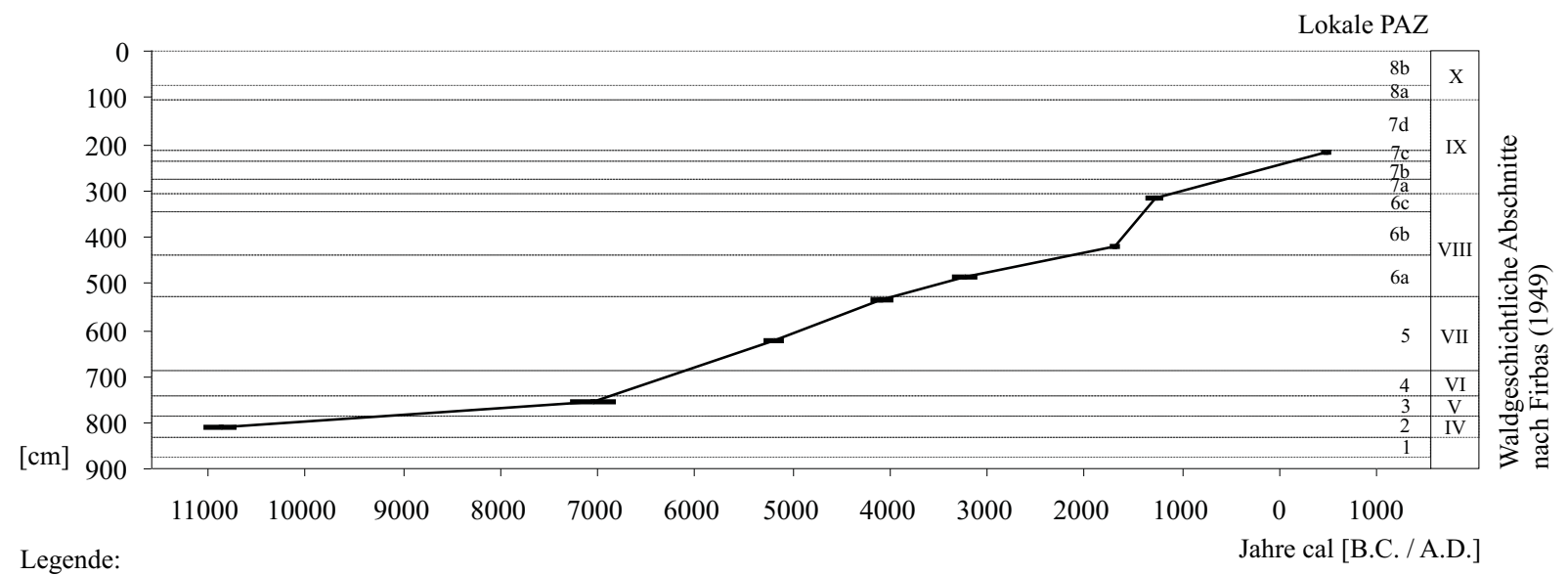

${ }^{\text {Legende: }}{ }^{14} \mathrm{C}$ Alter, kalibriert

Sedimentzuwachskurve nach linearer Interpolation

Abbildung 2: Zeit-Tiefen-Diagramm vom Profil Rybojady 


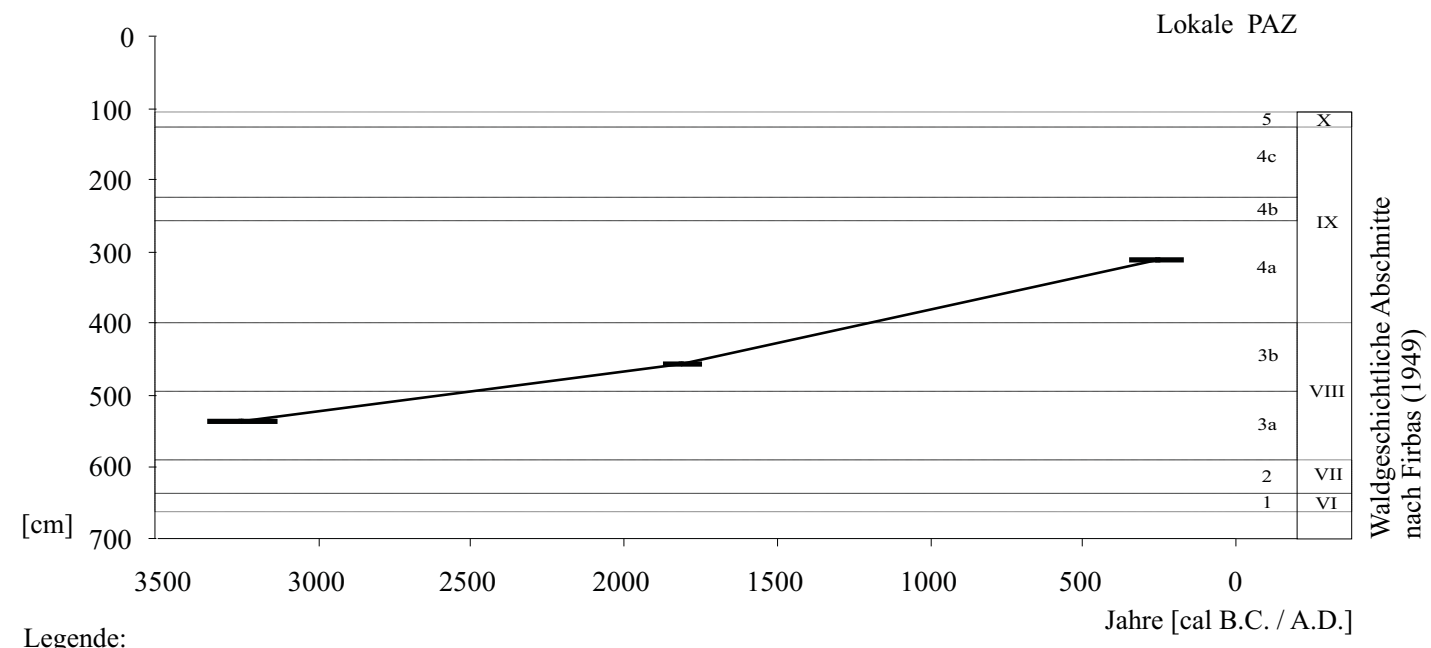

Legende:

Jahre [cal B.C. / A.D.]

- ${ }^{14} \mathrm{C}$ Alter, kalibriert

- Sedimentzuwachskurve nach linearer Interpolation

Abbildung 3: Zeit-Tiefen-Diagramm vom Profil Pawski Ług

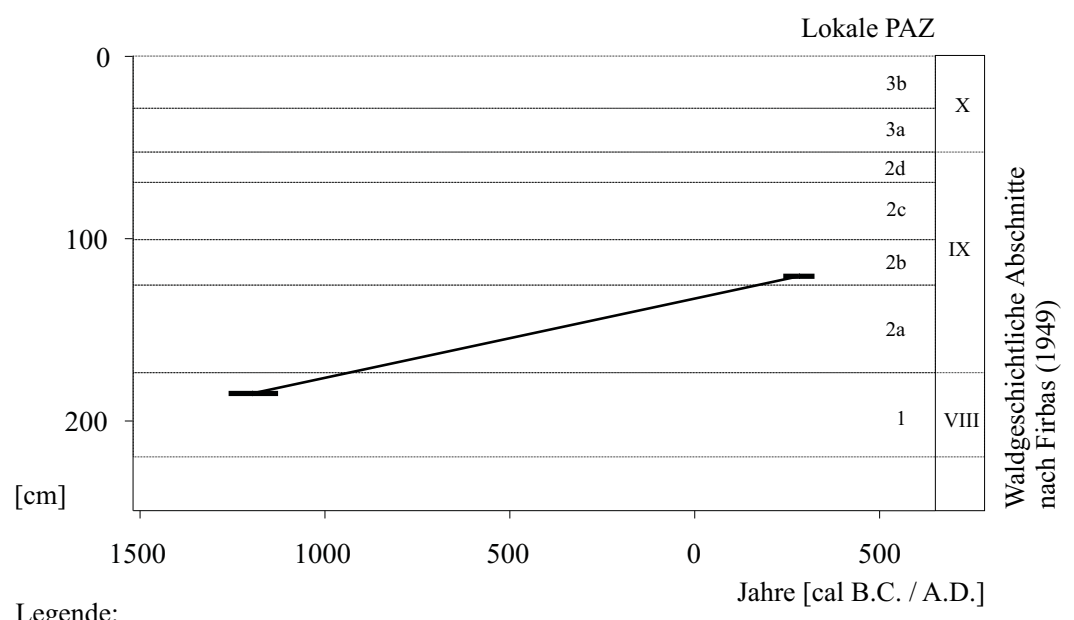

Legende:

- ${ }^{14} \mathrm{C}$ Alter, kalibriert

- Sedimentzuwachskurve nach linearer Interpolation

Abbildung 4: Zeit-Tiefen-Diagramm vom Profil Krebssee 


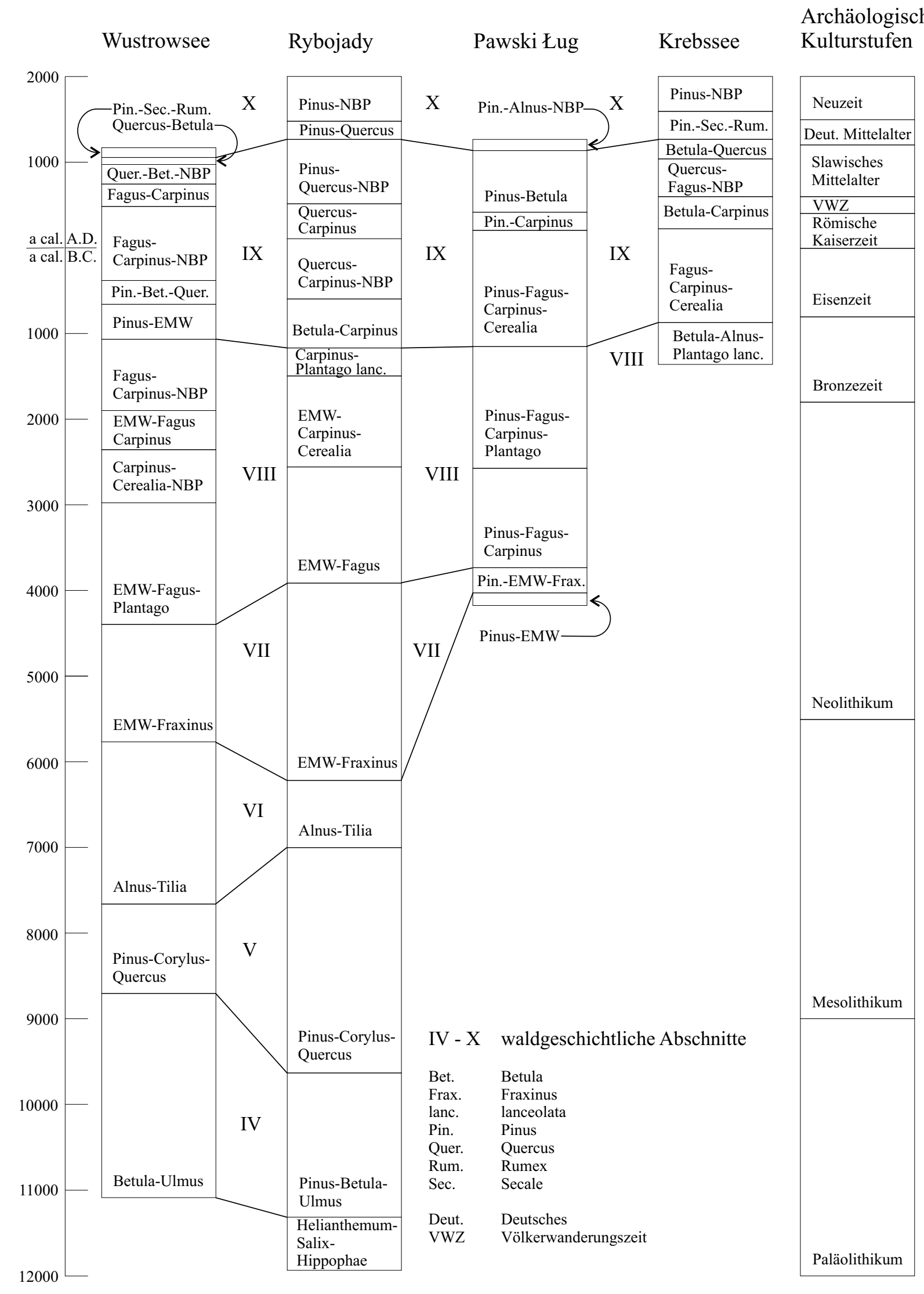

Abbildung 5: Synchronisation der L PAZ mit den waldgeschichtlichen Abschnitten und den archäologischen Kulturstufen 

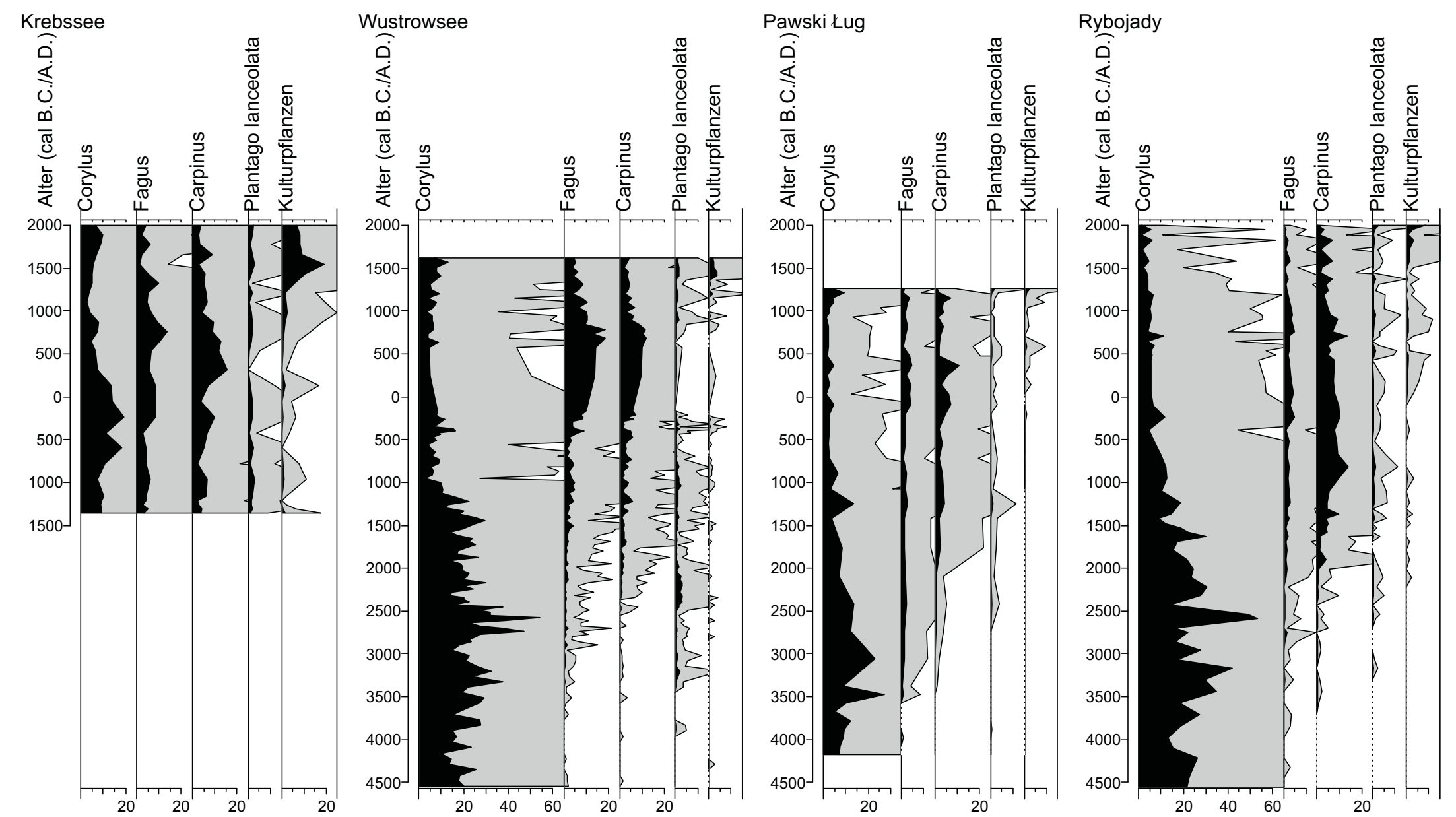

Abbildung 6: Die Einwanderung und Ausbreitung von Fagus und Carpinus im Zusammenhang mit dem Corylus-Rückgang und der Zunahme von Siedlungszeigern 


\section{Tiefe}

Beschreibung $[\mathrm{cm}]$

$\begin{array}{rrl}0 & 51 & \text { Kernverlust } \\ 51 & 95 & \text { schwarz-olive Sapropel } \\ 95 & 132 & \text { grau-olive FD-Mudde } \\ 132 & 395 & \text { olive FD-Mudde, lebermuddenartig } \\ 395 & 401 & \text { dunkelolive FD-Mudde } \\ 401 & 414,5 & \text { diffus laminierte olive FD-Mudde } \\ 414,5 & 444 & \text { schwarzbraune FD-Mudde } \\ 444 & 452 & \text { olive FD-Mudde } \\ 452 & 456 & \text { schwarzbraune FD-Mudde } \\ 456 & 758 & \text { olive KD-Mudde } \\ 758 & 867 & \text { dunkelolive FD-Mudde } \\ 867 & 978 & \text { dunkelolive KD-Mudde } \\ 978 & 1021,5 & \text { schwarzbraune FD-Mudde } \\ 1021,5 & 1039,5 & \text { laminierte olive FD-Mudde } \\ 1039,5 & 1075 & \text { diffus laminierte dunkelolive FD-Mudde } \\ 1075 & 1164 & \text { grauolive KD-Mudde } \\ 1164 & 1175 & \text { braunschwarze FD-Mudde } \\ 1175 & 1180 & \text { grauolive KD-Mudde } \\ 1180 & 1203 & \text { braunschwarze FD-Mudde } \\ 1203 & 1275 & \text { grauolive KD-Mudde } \\ 1275 & 1278 & \text { schwarze FD-Mudde } \\ 1278 & 1399 & \text { diffus laminierte olive KD-Mudde } \\ 1399 & 1458 & \text { dunkelolive KD-Mudde } \\ 1458 & 1515 & \text { diffus laminierte KD-Mudde } \\ 1515 & 1553 & \text { dunkelolive KD-Mudde }\end{array}$

Tabelle 1: Sedimentstratigraphie vom Profil Wustrowsee

Tiefe Beschreibung $[\mathrm{cm}]$

065 schwarzblaue, tonige KD-Mudde

65102 schwarze, torfige KD-Mudde

102137 olive KD-Mudde

137242 dunkelolive KD-Mudde

Tabelle 2: Sedimentstratigraphie vom Profil Krebssee 


\section{Tiefe Beschreibung}

$[\mathrm{cm}]$

0170 mäßig zersetzter Sphagnum-Torf

170210 Grobdetritusmudde

210310 dunkelbraune FD-Mudde

310500 hellbraune FD-Mudde, lebermuddenartig

500690 oliv-braune FD-Mudde

690794 braune FD-Mudde, lebermuddenartig

794810 hellbraune FD-Mudde, sandig

810835 Sand

835850 dunkelolive FD-Mudde

850860 hellbraune FD-Mudde

860883 Sand

Tabelle 3: Sedimentstratigraphie vom Profil Rybojady

\section{Tiefe Beschreibung} $[\mathrm{cm}]$

100150 mäßig zersetzter Sphagnumtorf

150220 Sphagnumtorf mit Eriophorumblattscheiden

220236 Sphagnumtorf

236261 Sphagnumtorf mit Eriophorumblattscheiden

261490 mäßig zersetzter Radizellentorf

490550 Radizellentorf

550565 Braunmoosradizellentorf

565620 Grobdetritusmudde mit Braunmoosen

620644 dunkelbraune Grobdetritusmudde mit Molluskenschill

644665 dunkelbraune FD-Mudde

Tabelle 4: Sedimentstratigraphie vom Profil Pawski Ług 


\begin{tabular}{|c|c|c|c|c|c|c|}
\hline rofil & $\begin{array}{l}\text { Tiefe } \\
\quad[\mathrm{cm}]\end{array}$ & Nr. & $\begin{array}{l}\mathrm{C} \\
{[\mathrm{mg}]}\end{array}$ & $\begin{array}{c}\delta^{13} \mathrm{C} \\
{[\% \circ]} \\
\end{array}$ & $\begin{array}{r}{ }^{14} \mathrm{C} \text { - Alter } \\
\text { [B.P.] }\end{array}$ & [cal B \\
\hline \multirow[t]{4}{*}{ WUS3 } & $1549-1554$ & KIA 15639 & 5,1 & $-28,77$ & $11040 \pm 45$ & $11192-11028$ \\
\hline & 1497-1499 & 167 & 5,3 & $-28,52$ & $9990 \pm 46$ & $9600-9313$ \\
\hline & $1445-$ & & 4,8 & $-27,25$ & 951 & 3742 \\
\hline & $1301-1302$ & 0021 & 4,0 & $-28,87$ & $8435 \pm 35$ & $7571-7482$ \\
\hline \multirow[t]{9}{*}{ WUS1 } & 1093-1094 & & 4,2 & $-28,21$ & $6840 \pm 30$ & $5740-5664$ \\
\hline & 930-931 & KIA & 2,7 & $-27,69$ & $5520 \pm 30$ & $4442-4335$ \\
\hline & 843-844 & KIA & 2,4 & $-24,08$ & $4845 \pm 30$ & $3659-3543$ \\
\hline & 731-732 & & 2,8 & $-26,98$ & & $3088-2924$ \\
\hline & $683-684$ & & 3,6 & $-25,88$ & 423 & -2714 \\
\hline & $451-452$ & & 5,5 & $-26,14$ & $3170 \pm 25$ & $1491-1410$ \\
\hline & $307-308,5$ & & 3,2 & $-25,95$ & $2380 \pm 35$ & $515-396$ \\
\hline & $227-229$ & KIA & 1,3 & $-28,47$ & $2110 \pm 30$ & $171-64$ \\
\hline & $211-212,5$ & KIA 16928 & 1,3 & $-29,76$ & $1375 \pm 25$ & 644-670 A.D. \\
\hline \multirow[t]{2}{*}{ KBS2 } & $185-186$ & KIA 20022 & 1,0 & $-29,94$ & $2970 \pm 35$ & $1260-1128$ \\
\hline & $121-122$ & KIA & 3,6 & $-32,16$ & $1755 \pm 20$ & 242-323 A.D. \\
\hline \multirow[t]{9}{*}{$\mathrm{R} 1.00$} & $854-866$ & & 1,8 & $-25,49$ & $18850 \pm 110$ & 20793 \\
\hline & $804-8$ & & 0,5 & $-30,93$ & $10810 \pm 120$ & 11052-10703 \\
\hline & $751-757$ & & 0,6 & $-27,39$ & $8070 \pm 70$ & $7282-6830$ \\
\hline & $622-630$ & KIA & 1,8 & $-22,10$ & $6245 \pm 35$ & $5296-5084$ \\
\hline & 534-542 & KIA 18788 & 0,5 & $-23,92$ & $5205 \pm 55$ & $4217-3958$ \\
\hline & $486-494$ & $\mathrm{KI}$ & 0,3 & $-27,95$ & $4530 \pm 80$ & $3359-3099$ \\
\hline & & & 0,8 & $-25,18$ & $3390 \pm 35$ & $1739-1635$ \\
\hline & & & 1,0 & $-25,08$ & $3010 \pm 30$ & $1368-1193$ \\
\hline & $218-222$ & KIA 16929 & 1,5 & $-25,87$ & $1600 \pm 25$ & 422-530 A.D. \\
\hline \multirow{3}{*}{ L1.00 } & 550 & & 4,3 & $-27,52$ & $4565 \pm 25$ & $3366-3138$ \\
\hline & $453-458$ & & 5,9 & $-26,76$ & $3475 \pm 25$ & $1876-1744$ \\
\hline & $310-315$ & KIA 19797 & 2,3 & $-29,36$ & $2165 \pm 25$ & $349-169$ \\
\hline
\end{tabular}

Tabelle 5: Meßdaten der Radiocarbonanalysen 
Lokale PAZ /

\begin{tabular}{|c|c|c|c|c|c|}
\hline Waldg & $\begin{array}{l}\text { geschichtlicher } \\
\text { Abschnitt }\end{array}$ & & $\begin{array}{l}\text { Alter } \\
\text { [cal A.D. / B.C.] }\end{array}$ & $\begin{array}{r}\text { Dauer } \\
{[\mathrm{a}]}\end{array}$ & Archäolog. Kulturstufe \\
\hline W 7 & Pinus-Secale-Rumex & $\mathrm{X}$ & $1160-1050$ & 110 & Deutsches Mittelalter \\
\hline W $6 \mathrm{f}$ & Quercus-Betula & IX & $1050-970$ & 80 & Slawisches Mittelalter \\
\hline W 6 e & Quercus-Betula-NBP & IX & $970-750$ & 220 & Slawisches Mittelalter \\
\hline W $6 \mathrm{~d}$ & Fagus-Carpinus & IX & $750-480$ & 270 & Slawisches Mittelalter / VWZ \\
\hline W $6 \mathrm{c}$ & Fagus-Carpinus-NBP & IX & 480 A.D.-390 B.C. & 870 & Röm. Kaiserzeit / Eisenzeit \\
\hline W $6 \mathrm{~b}$ & Pinus-Betula-Quercus & IX & $390-660$ & 270 & Eisenzeit \\
\hline W 6 a & Pinus-EMW & IX & $660-1060$ & 400 & Eisenzeit / Bronzezeit \\
\hline W $5 \mathrm{~d}$ & Fagus-Carpinus-NBP & VIII & $1060-1900$ & 840 & Bronzezeit \\
\hline W $5 \mathrm{c}$ & EMW-Fagus-Carpinus & VIII & $1900-2350$ & 450 & Neolithikum \\
\hline W 5 b & Carpinus-Cerealia-NBP & VIII & $2350-2980$ & 630 & Neolithikum \\
\hline W 5 a & EMW-Fagus-Plantago & VIII & $2980-4390$ & 1410 & Neolithikum \\
\hline W 4 & EMW-Fraxinus & VII & $4390-5760$ & 1370 & Neolithikum \\
\hline W 3 & Alnus-Tilia & VI & $5760-7660$ & 1900 & Mesolithikum \\
\hline W 2 & Pinus-Corylus-Quercus & $\mathrm{V}$ & $7660-8710$ & 1050 & Mesolithikum \\
\hline W 1 & Betula-Ulmus & IV & $8710-11080$ & 2360 & Spätpaläolithikum \\
\hline
\end{tabular}

VWZ Völkerwanderungszeit

Tabelle 6: Die lokalen PAZ vom Wustrowsee

Lokale PAZ / Waldgeschichtlicher

Abschnitt

K 3 b Pinus-NBP

K 3 a Pinus-Secale-Rumex

K 2 d Betula-Quercus

K 2 c Quercus-Fagus-NBP

K 2 b Betula-Carpinus

K 2 a Fagus-Carpinus-Cerealia

K 1 Betula-Alnus-Plantago lanceolata

VWZ Völkerwanderungszeit
Archäolog.

\section{Dauer Kulturstufe}

[cal A.D. / B.C.] [a]

$\begin{array}{rrrl}\text { X } & 1999-1600 & 399 & \text { Neuzeit } \\ \text { X } & 1600-1270 & 330 & \text { Deutsches Mittelalter } \\ \text { IX } & 1270-1040 & 230 & \text { Slawisches Mittelalter } \\ \text { IX } & 1040-590 & 450 & \text { Slawisches Mittelalter } \\ \text { IX } & 590-220 & 370 & \text { VWZ / Röm. Kaiserzeit } \\ \text { IX } & 220 \text { A.D.-870 B.C. } & 1090 & \text { Röm. Kaiserzeit / Eisenzeit } \\ \text { VIII } & 870-1360 & 490 & \text { Bronzezeit }\end{array}$

Tabelle 7: Die lokalen PAZ vom Krebssee 
Lokale PAZ / Waldgeschichtlicher

Abschnitt

Alter

Dauer Archäolog. Kulturstufe

[cal A.D. / B.C.] [a]

R 8 b Pinus-NBP

R 8 a Pinus-Quercus

R 7 d Pinus-Quercus-NBP

R 7 c Quercus-Carpinus

R 7 b Quercus-Carpinus-NBP

R 7 a Betula-Carpinus

R 6 c Carpinus-Plantago lanceolata

R 6 b EMW-Carpinus-Cerealia

R 6 a EMW-Fagus

R 5 EMW-Fraxinus

R 4 Alnus-Tilia

R 3 Pinus-Corylus-Quercus

R 2 Pinus-Betula-Ulmus

R 1 Helianthemum-Salix-Hippophae
$2001-1480$

$1480-1270$

$1270-510$

$510-110$

IX

IX

IX

VIII

VIII

VIII

VII

VI

$\mathrm{V}$

IV

110 A.D. - 600 B.C.

$600-1170$

1170 - 1500

1500 - 2560

2560 - 3920

$3920-6220$

$6220-7000$

7000 - 9630

$9630-11320$
521 Neuzeit

210 Deutsches Mittelalter

760 Slawisches Mittelalter

400 VWZ / Röm. Kaiserzeit

$11320-11930$
710 Röm. Kaiserzeit / Eisenzeit

570 Eisenzeit / Bronzezeit

330 Bronzezeit

1060 Bronzezeit / Neolithikum

1360 Neolithikum

2300 Neolithikum / Mesolithikum

780 Mesolithikum

2630 Mesolithikum

1690 Spätpaläolithikum

610 Spätpaläolithikum

Tabelle 8: Die lokalen PAZ von Rybojady

Lokale PAZ / Waldgeschichtlicher

Alter

Archäolog.

Abschnitt

[cal A.D. / B.C.]

Dauer Kulturstufe

\begin{tabular}{|c|c|c|c|c|c|}
\hline & Abschnitt & & [cal A.D. / B.C.] & [a] & \\
\hline P 5 & Betula-Alnus-NBP & $\mathrm{X}$ & $1270-1130$ & 140 & Deutsches Mittelalter \\
\hline P $4 \mathrm{c}$ & Pinus-Betula & IX & $1130-420$ & 710 & Slawisches Mittelalter \\
\hline P 4 b & Pinus-Carpinus & IX & $420-200$ & 220 & Völkerwanderungszeit \\
\hline P 4 a & Pinus-Fagus-Carpinus-Cerealia & IX & 200 A.D.-1160 B.C. & 1360 & Röm. Kaiserzeit / EZ / BZ \\
\hline P 3 b & Pinus-Fagus-Carpinus-Plantago & VIII & $1160-2570$ & 1410 & BZ / Neolithikum \\
\hline P 3 a & Pinus-Fagus-Carpinus & VIII & $2570-3730$ & 1160 & Neolithikum \\
\hline P 2 & Pinus-EMW-Fraxinus & VII & $3730-4030$ & 300 & Neolithikum \\
\hline P 1 & Pinus-EMW & VI & $4030-4180$ & 150 & Neolithikum \\
\hline
\end{tabular}

BZ Bronzezeit

EZ Eisenzeit

Tabelle 9: Die lokalen PAZ von Pawski Ług 


\begin{tabular}{lccrrrr} 
& IV/V & V/VI & VI/VII & VII/VIII & VIII/IX & IX/X \\
\hline & & & & & & \\
Wustrowsee & 8710 & 7660 & 5760 & 4390 & 1060 & 1050 \\
Krebssee & & & & & 870 & 1270 \\
Pawski Ług & & & 4030 & 3730 & 1160 & 1130 \\
Rybojady & 9630 & 7000 & 6220 & 3920 & 1170 & 1270
\end{tabular}

\begin{tabular}{|c|c|c|c|c|c|c|}
\hline Großer Krebssee & 8090 & 7460 & 5890 & 3940 & 830 & (Jahns 2000) \\
\hline Felchowsee & 7960 & 7130 & 5890 & 3890 & 930 & (Jahns 2000) \\
\hline Döbritzer Heide & 9050 & 7250 & & & & (Wolters 2002) \\
\hline Lednica-See & & & & ca. 4830 & & (Tobolski 1990) \\
\hline Swietokrzyskie-See & & ca. 7460 & & & & (Makohonienko 2001) \\
\hline Skrzetuszewo-See & & ca. 7540 & ca. 6390 & & & (Tobolski 1990) \\
\hline Sicienko & & ca. 6550 & & & & (Latałowa 2001) \\
\hline
\end{tabular}

Gościąż-See ca. 6250

UG Untersuchungsgebiet

Tabelle 10: Datierungen der Grenzen der waldgeschichtlichen Abschnitte

UG Waldgeschichtlicher Abschnitt [a]

\begin{tabular}{|c|c|c|c|c|c|c|c|}
\hline & IV & V & VI/VII & VIII & IX & $\mathrm{X}$ & \\
\hline Wustrowsee & & 1050 & 3270 & 3330 & 2110 & & \\
\hline Krebssee & & & & & 2140 & 740 & \\
\hline Pawski Ług & & & & & 2290 & & \\
\hline Rybojady & 1700 & 2630 & 3080 & 2750 & 2440 & 730 & \\
\hline Großer Krebssee & & 630 & 3520 & 3110 & & & (Jahns 2000) \\
\hline Felchowsee & & 830 & 3240 & 2960 & & & (Jahns 2000) \\
\hline Döbritzer Heide & & 1800 & & & & & (Wolters 2002) \\
\hline
\end{tabular}

UG Untersuchungsgebiet

Tabelle 11: Dauer der waldgeschichtlichen Abschnitte 
[B.P. a] [cal B.C. a] von [\%] nach [\%]

$\begin{array}{llrrrr}\text { 1 Wustrowsee } & \text { östl. unteres Odertal } & 5520 \pm 30 & 4350 & 7,1 & 3,8 \\ \text { 1 Pawski Ług } & \text { südl. unteres Wartatal } & * 4700 & * 3780 & 4,7 & 1,9 \\ \text { 1 Rybojady } & \text { südl. unteres Wartatal } & 5205 \pm 55 & 3980 & 9,5 & 5,9 \\ \text { 2 Großer Krebssee } & \text { westl. unteres Odertal } & * 5210 & * 3940 & 4,2 & 1,2 \\ \text { 2 Felchowsee } & \text { westl. unteres Odertal } & * 5094 & * 3890 & 5,8 & 2,5 \\ \text { 3 Berlin } & \text { Berlin/Brandenburg } & 5000 & 3800 & \text { k. A. } & \text { k. A. } \\ \text { 4 Fercher Berge } & \text { Brandenburg } & 5500 \pm 105 & 4470-4170 & \text { k. A. } & \text { k. A. } \\ \text { 5 Lednica-See } & \text { Wielkoposka-Kujawy } & 5950 \pm 120 & 4720-5050 & \text { k. A. } & \text { k. A. } \\ \text { 6 Skrzetuszewo-See } & \text { Wielkoposka-Kujawy } & 5250 \pm 110 & 3980-4240 & \text { k. A. } & \text { k. A. } \\ \text { 7 Gościażz-See } & \text { Zentralpolen } & & * 3950 & \text { k. A. } & \text { k. A. } \\ \text { 8 Wolin } & \text { Wolin } & 5860 \pm 110 & 4680 & \text { k. A. } & \text { k. A. }\end{array}$

$\begin{array}{lll}* \text { *interpoliert } & \text { 1 Herking (2004) } & 5 \text { Tobolski (1990) } \\ \text { k.A. keine Angabe } & \text { 2 Jahns (2000) } & 6 \text { Makohonienko (2001) } \\ & \text { 3 Brande (1990) } & 7 \text { Ralska-Jasiewiczowa et al. } \text { (1998) } \\ & 4 \text { Wolters }(1999) & \text { 8 Latałowa (1992) }\end{array}$

Tabelle 12: Die Datierungen des Ulmenfalles

UG emp. PG Fagus-Anteile an der Baumpollensumme

$>1 \%$ $>5 \% \quad>10 \%$

Wustrowsee $4340 \mathrm{cal}$ B.C. $2730 \mathrm{cal}$ B.C. $1930 \mathrm{cal}$ B.C. $1430 \mathrm{cal} \mathrm{B.C.} 400 \mathrm{cal} \mathrm{B.C.} 130 \mathrm{cal}$ B.C. Krebssee $\quad 1360 \mathrm{cal} \mathrm{B.C.} 1200 \mathrm{cal} \mathrm{B.C.} 960 \mathrm{cal} \mathrm{B.C.} 650 \mathrm{cal}$ A.D.

Pawski Ług $3470 \mathrm{cal}$ B.C. $3050 \mathrm{cal}$ B.C. $1240 \mathrm{cal}$ B.C. $90 \mathrm{cal} \mathrm{B.C.}$

Rybojady $\quad 3580 \mathrm{cal} \mathrm{B.C.} 2540 \mathrm{cal}$ B.C. $1480 \mathrm{cal}$ B.C. $810 \mathrm{cal} \mathrm{B.C.} 540 \mathrm{cal} \mathrm{A.D.}$

UG Untersuchungsgebiet

emp. PG empirische Pollengrenze

Tabelle 13: Altersangaben zur Einwanderung und Ausbreitung von Fagus

UG emp. PG Carpinus-Anteile an der Baumpollensumme

$\begin{array}{lllll}>1 \% & >2 \% & >4 \% & >5 \% & >10 \%\end{array}$

Wustrowsee $2900 \mathrm{cal}$ B.C. $2580 \mathrm{cal}$ B.C. $2130 \mathrm{cal}$ B.C. $1430 \mathrm{cal} \mathrm{B.C.} 460 \mathrm{cal} \mathrm{B.C.} 660$ cal A.D. Krebssee $1360 \mathrm{cal} \mathrm{B.C.} 1160 \mathrm{cal} \mathrm{B.C.} 1160 \mathrm{cal} \mathrm{B.C.} 310$ cal A.D. Pawski Ług $3370 \mathrm{cal} \mathrm{B.C.} 1770 \mathrm{cal} \mathrm{B.C.} 1420 \mathrm{cal} \mathrm{B.C.} 200 \mathrm{cal} \mathrm{B.C.} 200 \mathrm{cal} \mathrm{B.C.} 370$ cal A.D. Rybojady $2700 \mathrm{cal} \mathrm{B.C.} 2320 \mathrm{cal}$ B.C. $1900 \mathrm{cal}$ B.C. $1900 \mathrm{cal} \mathrm{B.C.} 1690 \mathrm{cal} \mathrm{B.C.} 1300 \mathrm{cal} \mathrm{B.C.}$

UG Untersuchungsgebiet

emp. PG empirische Pollengrenze

Tabelle 14: Altersangaben zur Einwanderung und Ausbreitung von Carpinus 


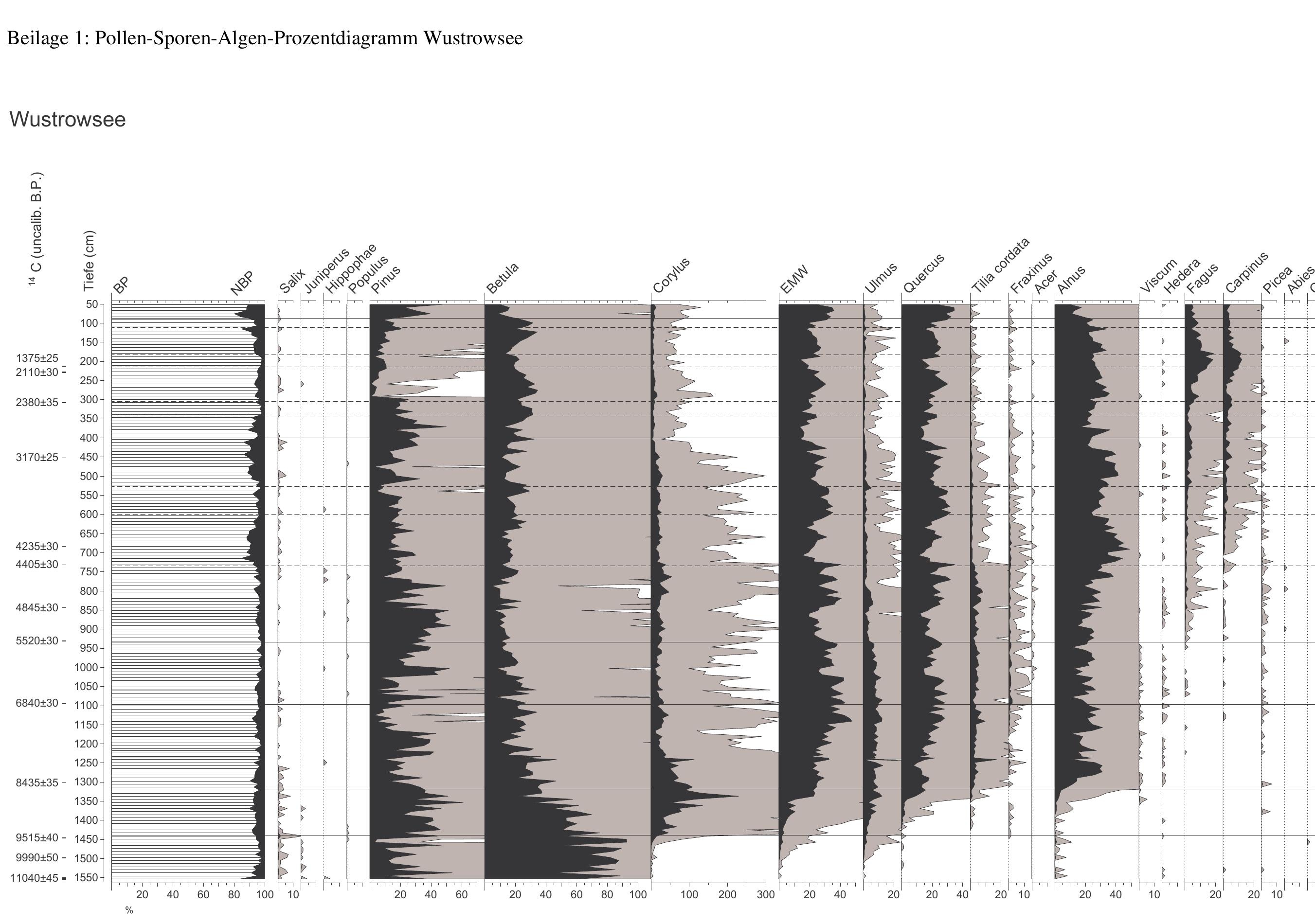




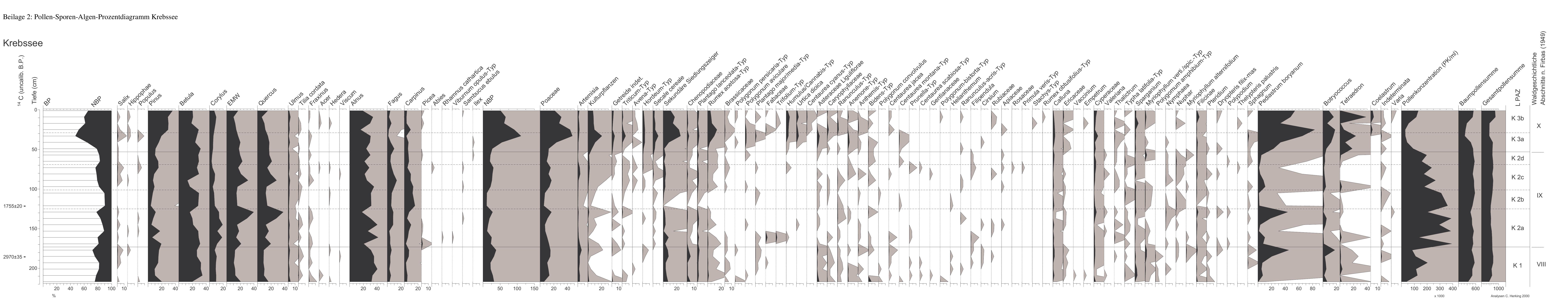




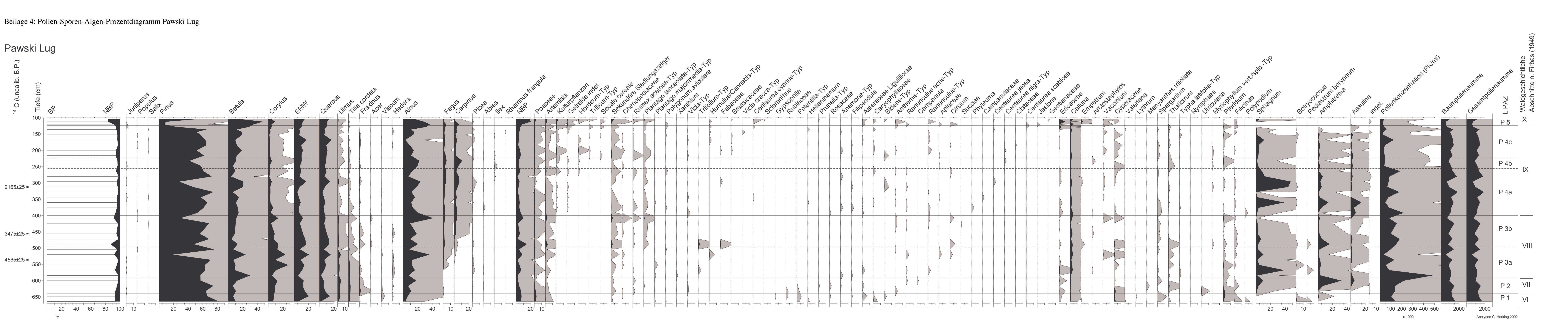

